JOURNAL OF THE

AMERICAN MATHEMATICAL SOCIETY

Volume 20, Number 3, July 2007, Pages 753-798

S 0894-0347(06)00551-0

Article electronically published on October 24, 2006

\title{
GLOBAL WELL-POSEDNESS OF THE BENJAMIN-ONO EQUATION IN LOW-REGULARITY SPACES
}

\author{
ALEXANDRU D. IONESCU AND CARLOS E. KENIG
}

\section{INTRODUCTION}

In this paper we consider the Benjamin-Ono initial-value problem

$$
\left\{\begin{array}{l}
\partial_{t} u+\mathcal{H} \partial_{x}^{2} u+\partial_{x}\left(u^{2} / 2\right)=0 \text { on } \mathbb{R}_{x} \times \mathbb{R}_{t} \\
u(0)=\phi
\end{array}\right.
$$

where $\mathcal{H}$ is the Hilbert transform operator defined (on the spaces $C\left(\mathbb{R}: H^{\sigma}\right), \sigma \in \mathbb{R}$ ) by the Fourier multiplier $-i \operatorname{sgn}(\xi)$. The Benjamin-Ono equation is a model for one-dimensional long waves in deep stratified fluids ([1] and [16]) and is completely integrable. The initial-value problem for this equation has been studied extensively for data in the Sobolev spaces $H_{r}^{\sigma}(\mathbb{R}), \sigma \geq 0 \mathbb{1}$ It is known that the Benjamin-Ono initial-value problem has weak solutions in $H_{r}^{0}(\mathbb{R}), H_{r}^{1 / 2}(\mathbb{R})$, and $H_{r}^{1}(\mathbb{R})$ (see [5], [25], and [18) and is globally well-posed in $H_{r}^{\sigma}(\mathbb{R}), \sigma \geq 1$ (see [22], as well as [7, [17, [12, and [8] for earlier local and global well-posedness results in higher regularity spaces). In this paper we prove that the Benjamin-Ono initial-value problem is globally well-posed in $H_{r}^{\sigma}(\mathbb{R}), \sigma \geq 0$.

Let $H_{r}^{\infty}(\mathbb{R})=\bigcap_{\sigma=0}^{\infty} H_{r}^{\sigma}(\mathbb{R})$ with the induced metric. Let $S^{\infty}: H_{r}^{\infty}(\mathbb{R}) \rightarrow C(\mathbb{R}:$ $H_{r}^{\infty}(\mathbb{R})$ ) denote the (nonlinear) mapping that associates to any data $\phi \in H_{r}^{\infty}$ the corresponding classical solution $u \in C\left(\mathbb{R}: H_{r}^{\infty}\right)$ of the initial-value problem (1.1). We will use the $L^{2}$ conservation law: if $\phi \in H_{r}^{\infty}$ and $u=S^{\infty}(\phi)$, then

$$
\int_{\mathbb{R}} u(x, t)^{2} d x=\int_{\mathbb{R}} \phi(x)^{2} d x \text { for any } t \in \mathbb{R} .
$$

For $T>0$ let $S_{T}^{\infty}: H_{r}^{\infty}(\mathbb{R}) \rightarrow C\left([-T, T]: H_{r}^{\infty}(\mathbb{R})\right)$ denote the restriction of the mapping $S^{\infty}$ to the time interval $[-T, T]$.

Received by the editors October 10, 2005.

2000 Mathematics Subject Classification. Primary 35Q53.

Key words and phrases. Benjamin-Ono initial-value problem, global well-posedness, local smoothing, gauge transformation.

The first author was supported in part by an NSF grant, a Sloan Research Fellowship, and a Packard Fellowship.

The second author was supported in part by an NSF grant.

${ }^{1}$ In this paper $H_{r}^{\sigma}=H_{r}^{\sigma}(\mathbb{R})$ denotes the space of real-valued functions $\phi$ with the usual norm $\|\phi\|_{H_{r}^{\sigma}}=\|\phi\|_{H^{\sigma}}=\left\|\left(1+|\xi|^{2}\right)^{\sigma / 2} \widehat{\phi}(\xi)\right\|_{L_{\xi}^{2}}$. All the other Banach spaces of functions, such as $L^{2}$, $H^{\sigma}, \widetilde{H}^{\sigma}, F^{\sigma}, N^{\sigma}$, etc., are defined as spaces of complex-valued functions.

(C)2006 American Mathematical Society Reverts to public domain 28 years from publication 
Theorem 1.1. (a) Assume $T>0$. Then the mapping $S_{T}^{\infty}: H_{r}^{\infty} \rightarrow C([-T, T]$ : $\left.H_{r}^{\infty}\right)$ extends uniquely to a continuous mapping $S_{T}^{0}: H_{r}^{0} \rightarrow C\left([-T, T]: H_{r}^{0}\right)$ and

$$
\left\|S_{T}^{0}(\phi)(t)\right\|_{H_{r}^{0}}=\|\phi\|_{H_{r}^{0}} \text { for any } t \in[-T, T], \phi \in H_{r}^{0} .
$$

The function $S_{T}^{0}(\phi)$ solves the initial-value problem (1.1) in $C\left([-T, T]: H_{r}^{-2}\right)$ for any $\phi \in H_{r}^{0}$.

(b) In addition, for any $\sigma \geq 0, S_{T}^{0}\left(H_{r}^{\sigma}\right) \subseteq C\left([-T, T]: H_{r}^{\sigma}\right)$,

$$
\left\|S_{T}^{0}(\phi)\right\|_{C\left([-T, T]: H_{r}^{\sigma}\right)} \leq C\left(T, \sigma,\|\phi\|_{H_{r}^{\sigma}}\right),
$$

and the mapping $S_{T}^{\sigma}=\left.S_{T}^{0}\right|_{H_{r}^{\sigma}}: H_{r}^{\sigma} \rightarrow C\left([-T, T]: H_{r}^{\sigma}\right)$ is continuous.

Clearly, if $T \leq T^{\prime}$ and $\phi \in H_{r}^{\sigma}$, then $S_{T}^{\sigma}(\phi)(t)=S_{T^{\prime}}^{\sigma}(\phi)(t)$ for any $t \in[-T, T]$. We mention that the flow map $\phi \rightarrow S_{T}^{\sigma}(\phi)$ fails to be uniformly continuous on bounded sets in $H_{r}^{\sigma}$ for any $T>0$ and $\sigma>0$; see [13. In a forthcoming paper [6] we prove a local well-posedness theorem for complex-valued data. See also the very recent papers [3] and [15] (which became available after the submission of this work) for other well-posedness results on the Benjamin-Ono equation and the periodic Benjamin-Ono equation.

We discuss now some of the ingredients in the proof of Theorem 1.1. The main obstruction to simply using bilinear estimates in some $X^{\sigma, b}$ space (in a way similar to the case of the KdV equation in [2] or nonlinear wave equations in [10]) is the lack of control of the interaction between very high and very low frequencies of solutions (cf. 14 and 13]). Following [22, we first construct a gauge transformation that weakens this interaction, in the sense that we will be able to assume that low frequency functions have some additional structure (see the space $Z_{0}$ defined in Section (3). Even with this low-frequency assumption, the use of standard $X^{\sigma, b}$ spaces for high-frequency functions (i.e., spaces defined by suitably weighted norms in the frequency space) seems to lead inevitably to logarithmic divergences in the modulation variable (see [4). To avoid these logarithmic divergences, we work with high-frequency spaces that have two components: an $X^{\sigma, b}$-type component measured in the frequency space and a normalized $L_{x}^{1} L_{t}^{2}$ component measured in the physical space. These types of spaces have been used in the context of wave maps (see, for example, [11, 23], 24], 19, and 20]); we remark that for the physical space component we use a suitable normalization of the local smoothing space $L_{x}^{1} L_{t}^{2}$ instead of the energy space $L_{t}^{1} L_{x}^{2}$. Then we prove suitable linear and bilinear estimates in these spaces and conclude the proof of Theorem 1.1 using a fixed-point argument.

The rest of the paper is organized as follows: in Section 2 we construct our gauge transformation and reduce solving the initial-value problem (1.1) to solving three easier initial-value problems. The point of this reduction is that the initial data of the resulting three initial-value problems have some special structure at very low frequencies (see the spaces $\widetilde{H}^{\sigma}$ defined in $(\underline{3.10})$ ). In Sections 3 and 4 we construct our main Banach spaces and prove some of their elementary properties. In Section 5 we prove several linear estimates using these Banach spaces. In Sections 6 , 7 and 8 we prove our main bilinear estimates. In Section 9 we prove several bounds for operators defined by multiplication with certain smooth bounded functions (such estimates are delicate in the context of $X^{\sigma, b}$ spaces). Finally, in Section 10 we combine all these estimates and a recursive argument to complete the proof of Theorem 1.1 . 


\section{The gauge transformation}

The first step is to construct a gauge transformation to weaken significantly the contribution coming from the low frequencies of the data. Assume $\phi \in H_{r}^{\infty}$ and $u=S^{\infty}(\phi) \in C\left(\mathbb{R}: H_{r}^{\infty}\right)$. On $L^{2}(\mathbb{R})$ we define the operators

$P_{\text {low }}$ defined by the Fourier multiplier $\xi \rightarrow \mathbf{1}_{\left[-2^{10}, 2^{10}\right]}(\xi)$;

$P_{ \pm \text {high }}$ defined by the Fourier multiplier $\xi \rightarrow \mathbf{1}_{\left[2^{10}, \infty\right)}( \pm \xi)$;

$P_{ \pm}$defined by the Fourier multiplier $\xi \rightarrow \mathbf{1}_{[0, \infty)}( \pm \xi)$.

Let $\phi_{0}=P_{\text {low }} \phi \in H_{r}^{\infty}, u_{0}=S^{\infty}\left(\phi_{0}\right), \widetilde{u}=u-u_{0}$. Since $\left\|\phi_{0}\right\|_{H_{r}^{\sigma}} \leq C_{\sigma}\|\phi\|_{L^{2}}$ for any $\sigma \geq 0$, it follows from the equation of $u_{0}$ that

$$
\sup _{t \in[-2,2]}\left\|\partial_{t}^{\sigma_{1}} \partial_{x}^{\sigma_{2}} u_{0}(., t)\right\|_{L_{x}^{2}} \leq C_{\sigma_{1}, \sigma_{2}}\|\phi\|_{L^{2}}, \sigma_{1}, \sigma_{2} \in[0, \infty) \cap \mathbb{Z} .
$$

Using the equation (1.1),

$$
\left\{\begin{array}{l}
\partial_{t} \widetilde{u}+\mathcal{H} \partial_{x}^{2} \widetilde{u}+\partial_{x}\left(u_{0} \cdot \widetilde{u}\right)+\partial_{x}\left(\widetilde{u}^{2} / 2\right)=0 \\
\widetilde{u}(0)=P_{+ \text {high }} \phi+P_{- \text {high }} \phi
\end{array}\right.
$$

We apply $P_{+ \text {high }}, P_{- \text {high }}$, and $P_{\text {low }}$ to (2.2) to obtain

$$
\left\{\begin{array}{l}
\partial_{t}\left(P_{ \pm h i g h} \widetilde{u}\right) \mp i \cdot \partial_{x}^{2}\left(P_{ \pm h i g h} \widetilde{u}\right)+P_{ \pm h i g h} \partial_{x}\left(u_{0} \cdot \widetilde{u}\right)+P_{ \pm h i g h} \partial_{x}\left(\widetilde{u}^{2} / 2\right)=0 \\
\left(P_{ \pm \operatorname{high}} \widetilde{u}\right)(0)=P_{ \pm h i g h} \phi
\end{array}\right.
$$

and

$$
\left\{\begin{array}{l}
\partial_{t}\left(P_{\text {low }} \widetilde{u}\right)+\mathcal{H} \partial_{x}^{2}\left(P_{\text {low }} \widetilde{u}\right)+P_{\text {low }} \partial_{x}\left(u_{0} \cdot \widetilde{u}\right)+P_{\text {low }} \partial_{x}\left(\widetilde{u}^{2} / 2\right)=0 \\
\left(P_{\text {low }} \widetilde{u}\right)(0)=0
\end{array}\right.
$$

We now let

$$
\left\{\begin{array}{l}
P_{+\operatorname{high}} \widetilde{u}=e^{-i U_{0}} w_{+} \\
P_{-\operatorname{hig}} \widetilde{u}=e^{i U_{0}} w_{-} \\
P_{\text {low }} \widetilde{u}=w_{0}
\end{array}\right.
$$

where $U_{0}$ is a suitable gauge that depends only on $u_{0}$. As in [22], we define first $U(0, t)$ on the time axis $x=0$ by the formula

$$
\partial_{t} U_{0}(0, t)+\frac{1}{2} \mathcal{H} \partial_{x} u_{0}(0, t)+\frac{1}{4} u_{0}^{2}(0, t)=0, \quad U_{0}(0,0)=0
$$

and then we construct $U_{0}(x, t)$ using the formula

$$
\partial_{x} U_{0}(x, t)=\frac{1}{2} u_{0}(x, t) .
$$

It is important to notice that $U_{0}$ is real-valued, since $\phi_{0}$ and $u_{0}$ are both real-valued.

Using the equation (1.1) for $u_{0}=S^{\infty}\left(\phi_{0}\right)$ and (2.7), we have

$$
\partial_{x}\left[\partial_{t} U_{0}+\mathcal{H} \partial_{x}^{2} U_{0}+\left(\partial_{x} U_{0}\right)^{2}\right]=0 \text { on } \mathbb{R} \times \mathbb{R} .
$$

Using (2.6) and (2.7) it follows that

$$
\partial_{t} U_{0}=-\frac{1}{2} \mathcal{H} \partial_{x} u_{0}-\frac{1}{4} u_{0}^{2} \text { on } \mathbb{R} \times \mathbb{R} .
$$


In particular, in view of (2.7) and (2.8), $U_{0} \in C^{\infty}(\mathbb{R} \times \mathbb{R})$. In fact, it follows from (2.1), (2.7), and (2.8) that for any integers $\sigma_{1}, \sigma_{2} \geq 0,\left(\sigma_{1}, \sigma_{2}\right) \neq(0,0)$,

$$
\sup _{t \in[-2,2]}\left\|\partial_{t}^{\sigma_{1}} \partial_{x}^{\sigma_{2}} U_{0}(., t)\right\|_{L_{x}^{2}} \leq C_{\sigma_{1}, \sigma_{2}}\|\phi\|_{L^{2}} .
$$

We substitute now the formulas $P_{+\operatorname{high}} \widetilde{u}=e^{-i U_{0}} w_{+}$and $\widetilde{u}=e^{-i U_{0}} w_{+}+e^{i U_{0}} w_{-}+$ $w_{0}$ in the equation (2.3) for $P_{+ \text {high }} \widetilde{u}$; the term $P_{+ \text {high }}\left(u_{0} e^{-i U_{0}} \partial_{x} w_{+}\right)$cancels (using (2.7)), and the result is

$$
\left\{\begin{array}{l}
\left(\partial_{t}+\mathcal{H} \partial_{x}^{2}\right) w_{+}=E_{+}\left(w_{+}, w_{-}, w_{0}\right) \\
w_{+}(0)=e^{i U_{0}(., 0)} P_{+\operatorname{high}} \phi
\end{array}\right.
$$

where

$$
\begin{aligned}
E_{+}\left(w_{+}, w_{-}, w_{0}\right)=-e^{i U_{0}} P_{+ \text {high }}\left[\partial_{x}\left(e^{-i U_{0}} w_{+}+e^{i U_{0}} w_{-}+w_{0}\right)^{2} / 2\right] \\
\quad-e^{i U_{0}} P_{+ \text {high }}\left[\partial_{x}\left[u_{0}\left(e^{i U_{0}} w_{-}+w_{0}\right)\right]\right] \\
\quad+e^{i U_{0}}\left(P_{- \text {high }}+P_{\text {low }}\right)\left(u_{0} e^{-i U_{0}} \partial_{x} w_{+}\right)+2 i P_{-}\left(\partial_{x}^{2} w_{+}\right) \\
\quad-e^{i U_{0}} P_{+ \text {high }}\left[\partial_{x}\left(u_{0} e^{-i U_{0}}\right) \cdot w_{+}\right]+i\left(\partial_{t} U_{0}-i \partial_{x}^{2} U_{0}-\left(\partial_{x} U_{0}\right)^{2}\right) \cdot w_{+} .
\end{aligned}
$$

Since $w_{+}=e^{i U_{0}} P_{+\operatorname{high}}\left(e^{-i U_{0}} w_{+}\right), w_{-}=e^{-i U_{0}} P_{-\operatorname{high}}\left(e^{i U_{0}} w_{-}\right)$, and $w_{0}=P_{\text {low }}\left(w_{0}\right)$ (see (2.5)), we use (2.7) and (2.8) to rewrite $E_{+}\left(w_{+}, w_{-}, w_{0}\right)$ in the form

$$
\begin{aligned}
E_{+}\left(w_{+},\right. & \left.w_{-}, w_{0}\right)=-e^{i U_{0}} P_{+ \text {high }}\left[\partial_{x}\left(e^{-i U_{0}} w_{+}+e^{i U_{0}} w_{-}+w_{0}\right)^{2} / 2\right] \\
& -e^{i U_{0}} P_{\text {+high }}\left[\partial_{x}\left[u_{0} \cdot P_{- \text {high }}\left(e^{i U_{0}} w_{-}\right)+u_{0} \cdot P_{\text {low }}\left(w_{0}\right)\right]\right] \\
& +e^{i U_{0}}\left(P_{- \text {high }}+P_{\text {low }}\right)\left[\partial_{x}\left(u_{0} \cdot P_{+ \text {high }}\left(e^{-i U_{0}} w_{+}\right)\right)\right] \\
& +2 i P_{-}\left[\partial_{x}^{2}\left(e^{i U_{0}} P_{+ \text {high }}\left(e^{-i U_{0}} w_{+}\right)\right)\right] \\
& -P_{+} \partial_{x} u_{0} \cdot w_{+} .
\end{aligned}
$$

A similar computation using the equation (2.3) for $P_{- \text {high }} \widetilde{u}$ gives

$$
\left\{\begin{array}{l}
\left(\partial_{t}+\mathcal{H} \partial_{x}^{2}\right) w_{-}=E_{-}\left(w_{+}, w_{-}, w_{0}\right) \\
w_{-}(0)=e^{-i U_{0}(., 0)} P_{-\operatorname{high}} \phi
\end{array}\right.
$$

where

$$
\begin{aligned}
E_{-}\left(w_{+},\right. & \left.w_{-}, w_{0}\right)=-e^{-i U_{0}} P_{- \text {high }}\left[\partial_{x}\left(e^{-i U_{0}} w_{+}+e^{i U_{0}} w_{-}+w_{0}\right)^{2} / 2\right] \\
& -e^{-i U_{0}} P_{-\operatorname{high}}\left[\partial_{x}\left[u_{0} \cdot P_{+ \text {high }}\left(e^{-i U_{0}} w_{+}\right)+u_{0} \cdot P_{\text {low }}\left(w_{0}\right)\right]\right] \\
& +e^{-i U_{0}}\left(P_{+ \text {high }}+P_{\text {low }}\right)\left[\partial_{x}\left(u_{0} \cdot P_{- \text {high }}\left(e^{i U_{0}} w_{-}\right)\right)\right] \\
& -2 i P_{+}\left[\partial_{x}^{2}\left(e^{-i U_{0}} P_{- \text {high }}\left(e^{i U_{0}} w_{-}\right)\right)\right] \\
& -P_{-} \partial_{x} u_{0} \cdot w_{-} .
\end{aligned}
$$

Finally, using (2.4),

$$
\left\{\begin{array}{l}
\left(\partial_{t}+\mathcal{H} \partial_{x}^{2}\right) w_{0}=E_{0}\left(w_{+}, w_{-}, w_{0}\right) \\
w_{0}(0)=0
\end{array}\right.
$$

where

$$
E_{0}\left(w_{+}, w_{-}, w_{0}\right)=-\frac{1}{2} P_{\text {low }}\left[\partial_{x}\left[\left(e^{-i U_{0}} w_{+}+e^{i U_{0}} w_{-}+w_{0}+u_{0}\right)^{2}-u_{0}^{2}\right]\right] .
$$

We summarize our construction in the following lemma. 
Lemma 2.1. Assume $\phi \in H_{r}^{\infty}$ and $u=S^{\infty}(\phi) \in C\left(\mathbb{R}: H_{r}^{\infty}\right)$. Then

$$
u=e^{-i U_{0}} w_{+}+e^{i U_{0}} w_{-}+w_{0}+u_{0}
$$

where $u_{0}=S^{\infty}\left(P_{\text {low }}(\phi)\right)$ satisfies (2.1), $U_{0}$ satisfies (2.9), and $w_{+}, w_{-}$, and $w_{0}$ satisfy the equations (2.10), (2.12), and (2.14), where $E_{+}, E_{-}$, and $E_{0}$ are as in (2.11), (2.13), and (2.15).

Remark. The expressions $E_{+}$and $E_{-}$in (2.11) and (2.13) appear complicated due to the various terms. We observe however that only the nonlinear terms in the first lines are difficult to handle: the terms in the second, third, and fourth lines are essentially of the form

$$
P_{ \pm}\left[\text {smooth function } \cdot P_{\mp}(\text { rough function })\right] \text {. }
$$

Such expressions have a strong smoothing effect on the rough function. Also, in the term in the fifth line, the derivative acts on the smooth function.

\section{The Banach SPACES}

In this section we construct our main resolution spaces. In view of the $L^{2}$ conservation law (1.2), it will suffice to construct the solution on the time interval $[-1,1]$. The resolution spaces we construct below are implicitly adapted to this restriction in time 2 The factor $i$ in (3.5), the restriction $j \geq 0$ in all the definitions, and the operators $I-\partial_{\tau}^{2}$ in (3.11) are related to the uncertainty principle satisfied by functions that are essentially supported in $\mathbb{R} \times[-1,1]$. This implicit time restriction, which is needed for the $L_{x}^{2} L_{t}^{\infty}$ bound in Lemma 4.2, creates a significant distinction between frequencies that are $\leq 1$ (for which the dispersive factor $\omega(\xi)$ is $\leq 1$, thus negligible in view of the uncertainty principle) and frequencies that are $\geq 1$. Our spaces reflect this distinction (see also the definitions of the sets $D_{k, j}$ and the factors $A_{k}$ below).

Let $\eta_{0}: \mathbb{R} \rightarrow[0,1]$ denote an even smooth function supported in $[-8 / 5,8 / 5]$ and equal to 1 in $[-5 / 4,5 / 4]$. For $l \in \mathbb{Z}$ let $\chi_{l}(\xi)=\eta_{0}\left(\xi / 2^{l}\right)-\eta_{0}\left(\xi / 2^{l-1}\right), \chi_{l}$ supported in $\left\{\xi:|\xi| \in\left[(5 / 8) \cdot 2^{l},(8 / 5) \cdot 2^{l}\right]\right\}$, and

$$
\chi_{\left[l_{1}, l_{2}\right]}=\sum_{l=l_{1}}^{l_{2}} \chi_{l} \text { for any } l_{1} \leq l_{2} \in \mathbb{Z} .
$$

For simplicity of notation, let $\eta_{l}=\chi_{l}$ if $l \geq 1$ and $\eta_{l} \equiv 0$ if $l \leq-1$. Also, for $l_{1} \leq l_{2} \in \mathbb{Z}$ let

$$
\eta_{\left[l_{1}, l_{2}\right]}=\sum_{l=l_{1}}^{l_{2}} \eta_{l} \text { and } \eta_{\leq l_{2}}=\sum_{l=-\infty}^{l_{2}} \eta_{l} .
$$

For any integer $k \geq 0$ and $\phi \in L^{2}(\mathbb{R})$ we define the operator $P_{k}$ by the formula

$$
\widehat{P_{k} \phi}(\xi)=\eta_{k}(\xi) \widehat{\phi}(\xi) \text {. }
$$

By a slight abuse of notation we also define the operators $P_{k}$ on $L^{2}(\mathbb{R} \times \mathbb{R})$ by the formula $\mathcal{F}\left(P_{k} u\right)(\xi, \tau)=\eta_{k}(\xi) \mathcal{F}(u)(\xi, \tau)$.

Let $\mathbb{Z}_{+}=\mathbb{Z} \cap[0, \infty)$. For $\xi \in \mathbb{R}$ let

$$
\omega(\xi)=-\xi|\xi| .
$$

\footnotetext{
${ }^{2}$ However, this time restriction is not exact; we do not multiply by cutoff functions in $t$ in the definition of the resolution spaces, since this would not be compatible with the atomic decompositions (4.1) and (4.2).
} 
For $l \in \mathbb{Z}$ let $I_{l}=\left\{\xi \in \mathbb{R}:|\xi| \in\left[2^{l-1}, 2^{l+1}\right]\right\}$. For $l \in[0, \infty) \cap \mathbb{Z}$ let $\widetilde{I}_{l}=[-2,2]$ if $l=0$ and $\widetilde{I}_{l}=I_{l}$ if $l \geq 1$. For $k \in \mathbb{Z}$ and $j \geq 0$ let

$$
\left\{\begin{array}{l}
D_{k, j}=\left\{(\xi, \tau) \in \mathbb{R} \times \mathbb{R}: \xi \in I_{k}, \tau-\omega(\xi) \in \widetilde{I}_{j}\right\} \text { if } k \geq 1 \\
D_{k, j}=\left\{(\xi, \tau) \in \mathbb{R} \times \mathbb{R}: \xi \in I_{k}, \tau \in \widetilde{I}_{j}\right\} \text { if } k \leq 0 .
\end{array}\right.
$$

We define first the Banach spaces $X_{k}=X_{k}(\mathbb{R} \times \mathbb{R}), k \in \mathbb{Z}_{+}$: for $k \geq 1$ we define

$$
\begin{aligned}
X_{k}= & \left\{f \in L^{2}: f \text { supported in } I_{k} \times \mathbb{R}\right. \text { and } \\
& \left.\|f\|_{X_{k}}:=\sum_{j=0}^{\infty} 2^{j / 2} \beta_{k, j}\left\|\eta_{j}(\tau-\omega(\xi)) f(\xi, \tau)\right\|_{L_{\xi, \tau}^{2}}<\infty\right\},
\end{aligned}
$$

where

$$
\beta_{k, j}=1+2^{(j-2 k) / 2} .
$$

The precise choice of the coefficients $\beta_{k, j}$ is important in order for all the bilinear estimates (7.1), (7.2), (8.1), and (8.2) to hold. Notice that $2^{j / 2} \beta_{k, j} \approx 2^{j}$ when $k$ is small. For $k=0$ we define

$$
\begin{aligned}
& X_{0}=\left\{f \in L^{2}: f \text { supported in } \widetilde{I}_{0} \times \mathbb{R}\right. \text { and } \\
&\left.\|f\|_{X_{0}}:=\sum_{j=0}^{\infty} \sum_{k^{\prime}=-\infty}^{1} 2^{j-k^{\prime}}\left\|\eta_{j}(\tau) \chi_{k^{\prime}}(\xi) f(\xi, \tau)\right\|_{L_{\xi, \tau}^{2}}<\infty\right\} .
\end{aligned}
$$

The spaces $X_{k}$ are not sufficient for our purpose, due to various logarithmic divergences. For $k \geq 100$ and $k=0$ we also define the Banach spaces $Y_{k}=Y_{k}(\mathbb{R} \times \mathbb{R})$. Let $\mathcal{F}$ and $\mathcal{F}_{1}$ denote the Fourier transform operators on $\mathcal{S}^{\prime}(\mathbb{R} \times \mathbb{R})$ and $\mathcal{S}^{\prime}(\mathbb{R})$, respectively. For $k \geq 100$ we define

$$
\begin{aligned}
& Y_{k}=\left\{f \in L^{2}: f \text { supported in } \bigcup_{j=0}^{k-1} D_{k, j}\right. \text { and } \\
& \left.\qquad\|f\|_{Y_{k}}:=2^{-k / 2}\left\|\mathcal{F}^{-1}[(\tau-\omega(\xi)+i) f(\xi, \tau)]\right\|_{L_{x}^{1} L_{t}^{2}}<\infty\right\} .
\end{aligned}
$$

For $k=0$ we define

$$
\begin{aligned}
& Y_{0}=\left\{f \in L^{2}: f \text { supported in } \widetilde{I}_{0} \times \mathbb{R}\right. \text { and } \\
& \left.\qquad\|f\|_{Y_{0}}:=\sum_{j=0}^{\infty} 2^{j}\left\|\mathcal{F}^{-1}\left[\eta_{j}(\tau) f(\xi, \tau)\right]\right\|_{L_{x}^{1} L_{t}^{2}}<\infty\right\} .
\end{aligned}
$$

Then we define

$$
Z_{k}:=X_{k} \text { if } 1 \leq k \leq 99 \text { and } Z_{k}:=X_{k}+Y_{k} \text { if } k \geq 100 \text { or } k=0 .
$$

The spaces $Z_{k}$ are our basic Banach spaces. The spaces $X_{k}$ are $X^{\sigma, b}$-type spaces; the spaces $Y_{k}$ are relevant due to the local smoothing inequality

$$
\left\|\partial_{x} u\right\|_{L_{x}^{\infty} L_{t}^{2}} \leq C\left\|\left(\partial_{t}+\mathcal{H} \partial_{x}^{2}\right) u\right\|_{L_{x}^{1} L_{t}^{2}} \text { for any } u \in \mathcal{S}(\mathbb{R} \times \mathbb{R}) .
$$

Remark. For $k \in[1,99] \cap \mathbb{Z}$ we could define the spaces $Y_{k}$ as in (3.5) and let $Z_{k}:=X_{k}+Y_{k}$. This is not necessary, however, in view of Lemma 4.1(b) below. 
In some estimates we will also need the space $\bar{Z}_{0}, Z_{0} \subseteq \bar{Z}_{0}$,

$$
\begin{gathered}
\bar{Z}_{0}=\left\{f \in L^{2}(\mathbb{R} \times \mathbb{R}): f \text { supported in } \widetilde{I}_{0} \times \mathbb{R}\right. \text { and } \\
\left.\|f\|_{\bar{Z}_{0}}:=\sum_{j=0}^{\infty} 2^{j}\left\|\eta_{j}(\tau) f(\xi, \tau)\right\|_{L_{\xi, \tau}^{2}}<\infty\right\} .
\end{gathered}
$$

We also define the space $B_{0}(\mathbb{R})$ by

$$
\begin{aligned}
B_{0}= & \left\{f \in L^{2}(\mathbb{R}): f \text { supported in } \widetilde{I}_{0}\right. \text { and } \\
& \left.\|f\|_{B_{0}}:=\inf _{f=g+h}\left\|\mathcal{F}_{1}^{-1}(g)\right\|_{L_{x}^{1}}+\sum_{k^{\prime}=-\infty}^{1} 2^{-k^{\prime}}\left\|\chi_{k^{\prime}} \cdot h\right\|_{L_{\xi}^{2}}<\infty\right\} .
\end{aligned}
$$

For $k \in \mathbb{Z}_{+}$let

$$
\left\{\begin{array}{l}
A_{k}(\xi, \tau)=\tau-\omega(\xi)+i \text { if } k \geq 1 \\
A_{k}(\xi, \tau)=\tau+i \text { if } k=0
\end{array}\right.
$$

For $\sigma \geq 0$ we define the Banach spaces $\widetilde{H}^{\sigma}=\widetilde{H}^{\sigma}(\mathbb{R}), F^{\sigma}=F^{\sigma}(\mathbb{R} \times \mathbb{R})$, and $N^{\sigma}=N^{\sigma}(\mathbb{R} \times \mathbb{R})$ :

$$
\widetilde{H}^{\sigma}=\left\{\phi \in L^{2}:\|\phi\|_{\widetilde{H}^{\sigma}}^{2}:=\left\|\eta_{0} \cdot \mathcal{F}_{1}(\phi)\right\|_{B_{0}}^{2}+\sum_{k=1}^{\infty} 2^{2 \sigma k}\left\|\eta_{k} \cdot \mathcal{F}_{1}(\phi)\right\|_{L^{2}}^{2}<\infty\right\},
$$

$$
F^{\sigma}=\left\{u \in \mathcal{S}^{\prime}(\mathbb{R} \times \mathbb{R}):\|u\|_{F^{\sigma}}^{2}:=\sum_{k=0}^{\infty} 2^{2 \sigma k}\left\|\eta_{k}(\xi)\left(I-\partial_{\tau}^{2}\right) \mathcal{F}(u)\right\|_{Z_{k}}^{2}<\infty\right\}
$$

and

$$
N^{\sigma}=\left\{u \in \mathcal{S}^{\prime}(\mathbb{R} \times \mathbb{R}):\|u\|_{N^{\sigma}}^{2}:=\sum_{k=0}^{\infty} 2^{2 \sigma k}\left\|\eta_{k}(\xi) A_{k}(\xi, \tau)^{-1} \mathcal{F}(u)\right\|_{Z_{k}}^{2}<\infty\right\} .
$$

\section{Properties of the spaces $Z_{k}$}

We start with some basic properties of the spaces $Z_{k}$. Using the definitions, if $k \geq 1$ and $f_{k} \in Z_{k}$, then $f_{k}$ can be written in the form

$$
\left\{\begin{array}{l}
f_{k}=\sum_{j=0}^{\infty} f_{k, j}+g_{k} \\
\sum_{j=0}^{\infty} 2^{j / 2} \beta_{k, j}\left\|f_{k, j}\right\|_{L^{2}}+\left\|g_{k}\right\|_{Y_{k}} \leq 2\left\|f_{k}\right\|_{Z_{k}},
\end{array}\right.
$$

such that $f_{k, j}$ is supported in $D_{k, j}$ and $g_{k}$ is supported in $\bigcup_{j=0}^{k-1} D_{k, j}$ (if $k \leq 99$, then $\left.g_{k} \equiv 0\right)$. If $f_{0} \in Z_{0}$, then $f_{0}$ can be written in the form

$$
\left\{\begin{array}{l}
f_{0}=\sum_{j=0}^{\infty} \sum_{k^{\prime}=-\infty}^{1} f_{0, j}^{k^{\prime}}+\sum_{j=0}^{\infty} g_{0, j} ; \\
\sum_{j=0}^{\infty} \sum_{k^{\prime}=-\infty}^{1} 2^{j-k^{\prime}}\left\|f_{0, j}^{k^{\prime}}\right\|_{L^{2}}+\sum_{j=0}^{\infty} 2^{j}\left\|\mathcal{F}^{-1}\left(g_{0, j}\right)\right\|_{L_{x}^{1} L_{t}^{2}} \leq 2\left\|f_{0}\right\|_{Z_{0}},
\end{array}\right.
$$

such that $f_{0, j}^{k^{\prime}}$ is supported in $D_{k^{\prime}, j}$ and $g_{0, j}$ is supported in $\widetilde{I}_{0} \times \widetilde{I}_{j}$. 
Lemma 4.1. (a) If $m, m^{\prime}: \mathbb{R} \rightarrow \mathbb{C}, k \geq 0$, and $f_{k} \in Z_{k}$, then

$$
\left\{\begin{array}{l}
\left\|m(\xi) f_{k}(\xi, \tau)\right\|_{Z_{k}} \leq C\left\|\mathcal{F}_{1}^{-1}(m)\right\|_{L^{1}(\mathbb{R})}\left\|f_{k}\right\|_{Z_{k}} \\
\left\|m^{\prime}(\tau) f_{k}(\xi, \tau)\right\| Z_{Z_{k}} \leq C\left\|m^{\prime}\right\|_{L^{\infty}(\mathbb{R})}\left\|f_{k}\right\|_{Z_{k}}
\end{array}\right.
$$

(b) If $k \geq 1, j \geq 0$, and $f_{k} \in Z_{k}$, then

$$
\left\|\eta_{j}(\tau-\omega(\xi)) f_{k}(\xi, \tau)\right\|_{X_{k}} \leq C|| f_{k} \|_{Z_{k}} .
$$

(c) If $k \geq 1, j \in[0, k]$, and $f_{k}$ is supported in $I_{k} \times \mathbb{R}$, then

$$
\left\|\mathcal{F}^{-1}\left[\eta_{\leq j}(\tau-\omega(\xi)) f_{k}(\xi, \tau)\right]\right\|_{L_{x}^{1} L_{t}^{2}} \leq C\left\|\mathcal{F}^{-1}\left(f_{k}\right)\right\|_{L_{x}^{1} L_{t}^{2}} .
$$

Proof of Lemma 4.1. Part (a) follows directly from Plancherel's theorem and the definitions.

For part (b), we may assume $k \geq 100, f_{k}=g_{k} \in Y_{k}$, and $j \leq k$. We notice that if $g_{k} \in Y_{k}$, then $g_{k}$ can be written in the form (4.6)

$$
\left\{\begin{array}{l}
g_{k}(\xi, \tau)=2^{k / 2} \chi_{[k-1, k+1]}(\xi)(\tau-\omega(\xi)+i)^{-1} \eta_{\leq k}(\tau-\omega(\xi)) \int_{\mathbb{R}} e^{-i x \xi} h(x, \tau) d x \\
\left\|g_{k}\right\|_{Y_{k}}=C\|h\|_{L_{x}^{1} L_{\tau}^{2}} .
\end{array}\right.
$$

The inequality in part (b) follows easily since $\left|\left\{\xi \in I_{k}:|\tau-\omega(\xi)| \leq 2^{j+1}\right\}\right| \leq C 2^{j-k}$; see (3.1).

For part (c), using Plancherel's theorem, it suffices to prove that

$$
\left\|\int_{\mathbb{R}} e^{i x \xi} \chi_{[k-1, k+1]}(\xi) \eta_{\leq j}(\tau-\omega(\xi)) d \xi\right\|_{L_{x}^{1} L_{\tau}^{\infty}} \leq C .
$$

In proving (4.7), we may assume $k \geq 100$. Then the function in the left-hand side of (4.7) is not zero only if $\tau \approx 2^{2 k}$. Simple estimates using the change of variable $\tau-\omega(\xi)=\alpha$ and integration by parts show that

$$
\left|\int_{\mathbb{R}} e^{i x \xi} \chi_{[k-1, k+1]}(\xi) \eta_{\leq j}(\tau-\omega(\xi)) d \xi\right| \leq C \frac{2^{j-k}}{1+\left(2^{j-k} x\right)^{2}}
$$

if $\tau \approx 2^{2 k}$, which suffices to prove [4.7).

Using (4.1) and Lemma 4.1(b), (c), it follows easily (see the proof of Lemma 5.2 for a similar argument) that if $k \geq 1$ and $\left(I-\partial_{\tau}^{2}\right) f_{k} \in Z_{k}$, then $f_{k}$ can be written in the form

$$
\left\{\begin{array}{l}
f_{k}=\sum_{j=0}^{\infty} f_{k, j}+g_{k} \\
\sum_{j=0}^{\infty} 2^{j / 2} \beta_{k, j}\left\|\left(I-\partial_{\tau}^{2}\right) f_{k, j}\right\|_{L^{2}}+\left\|\left(I-\partial_{\tau}^{2}\right) g_{k}\right\|_{Y_{k}} \leq C\left\|\left(I-\partial_{\tau}^{2}\right) f_{k}\right\|_{Z_{k}},
\end{array}\right.
$$

such that $f_{k, j}$ is supported in $D_{k, j}$ and $g_{k}$ is supported in $\bigcup_{j=0}^{k-20} D_{k, j}$ (if $k \leq 99$, then $\left.g_{k} \equiv 0\right)$. We prove now several estimates using the spaces $Z_{k}$.

Lemma 4.2. (a) If $k \geq 0, t \in \mathbb{R}$, and $f_{k} \in Z_{k}$, then

$$
\left\{\begin{array}{l}
\left\|\int_{\mathbb{R}} f_{k}(\xi, \tau) e^{i t \tau} d \tau\right\|_{L_{\xi}^{2}} \leq C\left\|f_{k}\right\|_{Z_{k}} \text { if } k \geq 1 \\
\left\|\int_{\mathbb{R}} f_{0}(\xi, \tau) e^{i t \tau} d \tau\right\|_{B_{0}} \leq C\left\|f_{0}\right\|_{Z_{0}} \text { if } k=0
\end{array}\right.
$$

As a consequence,

$$
F^{\sigma} \subseteq C\left(\mathbb{R}: \widetilde{H}^{\sigma}\right) \text { for any } \sigma \geq 0
$$


(b) If $k \geq 1$ and $\left(I-\partial_{\tau}^{2}\right) f_{k} \in Z_{k}$, then

$$
\left\|\mathcal{F}^{-1}\left(f_{k}\right)\right\|_{L_{x}^{2} L_{t}^{\infty}} \leq C 2^{k / 2}\left\|\left(I-\partial_{\tau}^{2}\right) f_{k}\right\|_{Z_{k}} .
$$

(c) If $k \geq 1$ and $f_{k} \in Z_{k}$, then

$$
\left\|\mathcal{F}^{-1}\left(f_{k}\right)\right\|_{L_{x}^{\infty} L_{t}^{2}} \leq C 2^{-k / 2}\left\|f_{k}\right\|_{Z_{k}} .
$$

Proof of Lemma 4.2, For part (a), $k \geq 1$, we use the representation (4.1). Assume first that $f_{k}=f_{k, j}$. Then

$$
\left\|\int_{\mathbb{R}} f_{k, j}(\xi, \tau) e^{i t \tau} d \tau\right\|_{L_{\xi}^{2}} \leq C\left\|f_{k, j}(\xi, \tau)\right\|_{L_{\xi}^{2} L_{\tau}^{1}} \leq C 2^{j / 2}\left\|f_{k, j}\right\|_{L_{\xi, \tau}^{2}},
$$

which proves (4.9) in this case.

Assume now that $k \geq 100, f_{k}=g_{k} \in Y_{k}$, and write $g_{k}$ as in (4.6). We define the modified Hilbert transform operator

$$
\mathcal{L}_{k}(g)(\mu)=\int_{\mathbb{R}} g(\tau)(\tau-\mu+i)^{-1} \eta_{\leq k}(\tau-\mu) d \tau, g \in L^{2}(\mathbb{R}) .
$$

Clearly, $\left\|\mathcal{L}_{k}\right\|_{L^{2} \rightarrow L^{2}} \leq C$, uniformly in $k$. We examine the formula (4.6) and let $h^{*}(x, \mu)=\mathcal{L}_{k}\left[e^{i t \tau} h(x, \tau)\right](\mu),\left\|h^{*}\right\|_{L_{x}^{1} L_{\mu}^{2}} \leq C\|h\|_{L_{x}^{1} L_{\tau}^{2}}$. Then, using (4.6), the Minkowski inequality, and a change of variables,

$$
\begin{aligned}
\left\|\int_{\mathbb{R}} g_{k}(\xi, \tau) e^{i t \tau} d \tau\right\|_{L_{\xi}^{2}} & \leq C 2^{k / 2}\left\|\chi_{[k-1, k+1]}(\xi) \int_{\mathbb{R}} e^{-i x \xi} h^{*}(x, \omega(\xi)) d x\right\|_{L_{\xi}^{2}} \\
& \leq C 2^{k / 2} \cdot 2^{-k / 2}\left\|h^{*}\right\|_{L_{x}^{1} L_{\mu}^{2}} \\
& \leq C\left\|g_{k}\right\|_{Y_{k}},
\end{aligned}
$$

which completes the proof of (4.9) in the case $k \geq 1$.

Assume now $k=0$. We use the representation (4.2). Assume first that $f_{0}=f_{0, j}^{k^{\prime}}$ is supported in $D_{k^{\prime}, j},\left\|f_{0}\right\|_{Z_{0}} \approx 2^{j-k^{\prime}}\left\|f_{0, j}^{k^{\prime}}\right\|_{L^{2}}$. Then

$$
\left\|\int_{\mathbb{R}} f_{0, j}^{k^{\prime}}(\xi, \tau) e^{i t \tau} d \tau\right\|_{B_{0}} \leq C 2^{-k^{\prime}}\left\|\int_{\mathbb{R}}\left|f_{0, j}^{k^{\prime}}(\xi, \tau)\right| d \tau\right\|_{L_{\xi}^{2}} \leq C 2^{-k^{\prime}} 2^{j / 2}\left\|f_{0, j}^{k^{\prime}}\right\|_{L^{2}},
$$

which suffices.

Assume now that $f_{0}=g_{0, j}$ is supported in $\widetilde{I}_{0} \times \widetilde{I}_{j},\left\|f_{0}\right\|_{Z_{0}} \approx 2^{j}\left\|\mathcal{F}^{-1}\left(g_{0, j}\right)\right\|_{L_{x}^{1} L_{t}^{2}}$. Then

$$
\left\|\int_{\mathbb{R}} g_{0, j}(\xi, \tau) e^{i t \tau} d \tau\right\|_{B_{0}} \leq C\left\|\mathcal{F}^{-1}\left(g_{0, j}\right)\right\|_{L_{x}^{1} L_{t}^{\infty}} \leq C 2^{j / 2}\left\|\mathcal{F}^{-1}\left(g_{0, j}\right)\right\|_{L_{x}^{1} L_{t}^{2}},
$$

which completes the proof of part (a).

For part (b) we use the representation (4.8). Assume first that $f_{k}=f_{k, j}$ and let $f_{k, j}^{\#}(\xi, \mu)=f_{k, j}(\xi, \mu+\omega(\xi))$. By integration by parts, the left-hand side of (4.11) is dominated by

$$
\sum_{n \in \mathbb{Z}} \frac{C}{n^{2}+1} \int_{\widetilde{I}_{j}}\left\|\int_{\mathbb{R}}\left(I-\partial_{\mu}^{2}\right) f_{k, j}^{\#}(\xi, \mu) e^{i x \xi} e^{i t \omega(\xi)} d \xi\right\|_{L_{x}^{2} L_{t \in[n-1 / 2, n+1 / 2]}^{\infty}} d \mu .
$$

The bound (4.11) now follows from the standard maximal function estimate

$$
\left\|\int_{\mathbb{R}} g(\xi) e^{i x \xi} e^{i t \omega(\xi)} d \xi\right\|_{L_{x}^{2} L_{t \in[-1 / 2,1 / 2]}^{\infty}} \leq C 2^{k / 2}\|g\|_{L_{\xi}^{2}}
$$


for any function $g$ supported in $I_{k}$; see [9, Theorem 2.7]. In fact, the argument above and (4.15) show that if $f_{k} \in X_{k}$, then

$$
\left\|\int_{\mathbb{R}^{2}} f_{k}(\xi, \tau) e^{i x \xi} e^{i t \tau} d \xi d \tau\right\|_{L_{x}^{2} L_{t \in[-1 / 2,1 / 2]}^{\infty}} \leq C 2^{k / 2}\left\|f_{k}\right\|_{X_{k}} .
$$

Remark. The inequality (4.11) is relevant only when $j \leq k$. For $j \geq k$ the Sobolev imbedding theorem easily gives a stronger estimate.

Assume now that $k \geq 100, f_{k}=g_{k},\left(I-\partial_{\tau}^{2}\right) g_{k} \in Y_{k}$. By integration by parts, the left-hand side of (4.11) is dominated by

$$
\sum_{n \in \mathbb{Z}} \frac{C}{n^{2}+1}\left\|\int_{\mathbb{R}^{2}}\left(I-\partial_{\tau}^{2}\right) g_{k}(\xi, \tau) e^{i x \xi} e^{i t \tau} d \xi d \tau\right\|_{L_{x}^{2} L_{t \in[n-1 / 2, n+1 / 2]}^{\infty}} .
$$

We write now $\left(I-\partial_{\tau}^{2}\right) g_{k}$ as in (4.6). In view of (4.6) and the Minkowski inequality (notice that $\left(I-\partial_{\tau}^{2}\right) g_{k}$ can be thought of as a superposition in $y_{0}$ of functions of the form $\left.2^{k / 2} \chi_{[k-1, k+1]}(\xi)(\tau-\omega(\xi)+i)^{-1} \eta_{\leq k}(\tau-\omega(\xi)) \cdot e^{-i y_{0} \xi} h\left(y_{0}, \tau\right)\right)$, it suffices to prove that if

$$
f(\xi, \tau)=2^{k / 2} \chi_{[k-1, k+1]}(\xi)(\tau-\omega(\xi)+i)^{-1} \eta_{\leq k}(\tau-\omega(\xi)) \cdot h(\tau),
$$

then

$$
\left\|\int_{\mathbb{R}^{2}} f(\xi, \tau) e^{i x \xi} e^{i t \tau} d \xi d \tau\right\|_{L_{x}^{2} L_{t \in[-1 / 2,1 / 2]}^{\infty}} \leq C 2^{k / 2}\|h\|_{L^{2}}
$$

Since $k \geq 100$ and $|\xi| \in\left[2^{k-2}, 2^{k+2}\right]$, we may assume that the function $h$ in (4.17) is supported in the set $\left\{\tau:|\tau| \in\left[2^{2 k-10}, 2^{2 k+10}\right]\right\}$. Let $h_{+}=h \cdot \mathbf{1}_{[0, \infty)}, h_{-}=h \cdot \mathbf{1}_{(-\infty, 0]}$, and define the corresponding functions $f_{+}$and $f_{-}$as in (4.17). By symmetry, it suffices to prove the bound (4.18) for the function $f_{+}$, which is supported in the set $\left\{(\xi, \tau): \xi \in\left[-2^{k+2},-2^{k-2}\right], \tau \in\left[2^{2 k-10}, 2^{2 k+10}\right]\right\}$. In view of (3.1),$\tau-\omega(\xi)=\tau-\xi^{2}$ on the support of $f_{+}$, and $f_{+}(\xi, \tau)=0$ unless $|\sqrt{\tau}+\xi| \leq C$. Let (by freezing $\xi=-\sqrt{\tau})$

$$
\begin{gathered}
f_{+}^{\prime}(\xi, \tau)=2^{k / 2} \chi_{[k-1, k+1]}(-\sqrt{\tau})\left(\tau-\xi^{2}+(\sqrt{\tau}+\xi)^{2}+i \sqrt{\tau} 2^{-k}\right)^{-1} \\
\times \eta_{0}(\sqrt{\tau}+\xi) \cdot h_{+}(\tau) .
\end{gathered}
$$

Simple estimates show that, with $\mu=\left|\tau-\xi^{2}\right|+1$,

$$
\left|f_{+}(\xi, \tau)-f_{+}^{\prime}(\xi, \tau)\right| \leq C 2^{k / 2}\left|h_{+}(\tau)\right| \frac{\eta_{\leq k+5}(\mu)}{\mu}\left(\frac{1}{\mu}+\frac{\mu}{2^{k}}\right) .
$$

As in the proof of Lemma 4.1(b), it follows that

$$
\left\|f_{+}-f_{+}^{\prime}\right\|_{X_{k}} \leq C\left\|h_{+}\right\|_{L^{2}} .
$$

Thus, using (4.16),$\left\|\mathcal{F}^{-1}\left(f_{+}-f_{+}^{\prime}\right)\right\|_{L_{x}^{2} L_{t \in[-1 / 2,1 / 2]}^{\infty}} \leq C 2^{k / 2}\left\|h_{+}\right\|_{L^{2}}$. To estimate $\|\left.\mathcal{F}^{-1}\left(f_{+}^{\prime}\right)\right|_{L_{x}^{2} L_{t \in[-1 / 2,1 / 2]}^{\infty}}$, we make the change of variables $\xi=-\sqrt{\tau}+\mu$. Then

$$
\begin{aligned}
\mathcal{F}^{-1}\left(f_{+}^{\prime}\right)(x, t)=2^{k / 2} \int_{\mathbb{R}} h_{+}(\tau) & (2 \sqrt{\tau})^{-1} \chi_{[k-1, k+1]}(-\sqrt{\tau}) e^{i t \tau} e^{-i x \sqrt{\tau}} d \tau \\
& \times \int_{\mathbb{R}} \eta_{0}(\mu)\left(\mu+i / 2^{k+1}\right)^{-1} e^{i x \mu} d \mu .
\end{aligned}
$$

The absolute value of the integral in $\mu$ in (4.20) is bounded by $C$. We make the change of variables $\tau=\theta^{2}$ in the first integral and use the bound (4.15). It follows that $\left\|\mathcal{F}^{-1}\left(f_{+}^{\prime}\right)\right\|_{L_{x}^{2} L_{t \in[-1 / 2,1 / 2]}^{\infty}} \leq C 2^{k / 2}\left\|h_{+}\right\|_{L^{2}}$, which completes the proof of (4.18). 
For part (c) we use the representation (4.1). Assume first that $f_{k}=f_{k, j}$ and let $f_{k, j}^{\#}(\xi, \mu)=f_{k, j}(\xi, \mu+\omega(\xi))$. It suffices to prove the stronger bound

$$
\left\|\int_{D_{k, j}} f_{k, j}(\xi, \tau) e^{i x_{0} \xi} e^{i t \tau} d \xi d \tau\right\|_{L_{t}^{2}} \leq C 2^{-k / 2} 2^{j / 2}\left\|f_{k, j}\right\|_{L^{2}},
$$

for any $x_{0} \in \mathbb{R}$. Using Plancherel's theorem, duality, and the Hölder inequality, the left-hand side of the inequality above is dominated by

$$
\begin{aligned}
& C \sup _{\|h\| \|_{L^{2}(\mathbb{R})=1}} \int_{I_{k} \times \widetilde{I}_{j}}\left|f_{k, j}^{\#}(\xi, \mu)\right| \cdot|h(\mu+\omega(\xi))| d \xi d \mu \\
& \leq C \sup _{\|h\|_{L^{2}(\mathbb{R})=1}} \int_{\widetilde{I}_{j}}\left(\int_{I_{k}}\left|f_{k, j}^{\#}(\xi, \mu)\right|^{2} d \xi\right)^{1 / 2}\left(\int_{I_{k}}|h(\mu+\omega(\xi))|^{2} d \xi\right)^{1 / 2} d \mu \\
& \leq C 2^{-k / 2} 2^{j / 2}\left(\int_{I_{k} \times \widetilde{I}_{j}}\left|f_{k, j}^{\#}(\xi, \mu)\right|^{2} d \xi d \mu\right)^{1 / 2},
\end{aligned}
$$

as desired.

Assume now that $k \geq 100, f_{k}=g_{k} \in Y_{k}$ and write $g_{k}$ as in (4.6). Using Plancherel's theorem and the Minkowski inequality (see the explanation preceding (4.17)), it suffices to prove that

$$
\left|\int_{\mathbb{R}} e^{i x_{0} \xi} \chi_{[k-1, k+1]}(\xi)(\tau-\omega(\xi)+i)^{-1} \eta_{\leq k}(\tau-\omega(\xi)) d \xi\right| \leq C 2^{-k}
$$

uniformly in $x_{0}$ and $\tau$ (assuming $k \geq 100$ ). We may assume $|\tau| \in\left[2^{2 k-10}, 2^{2 k+10}\right]$ and, by symmetry, $\tau \geq 0$. Then the variable $\xi$ in the integral in (4.21) is in the interval $[-\sqrt{\tau}-C,-\sqrt{\tau}+C]$ and $\tau-\omega(\xi)=\tau-\xi^{2}$. As in part (b), see (4.19), we replace the integrand $\mathbf{1}_{(-\infty, 0]}(\xi) \chi_{[k-1, k+1]}(\xi)\left(\tau-\xi^{2}+i\right)^{-1} \eta_{\leq k}\left(\tau-\xi^{2}\right)$ with $\chi_{[k-1, k+1]}(-\sqrt{\tau})\left(\tau-\xi^{2}+(\sqrt{\tau}+\xi)^{2}+i \sqrt{\tau} 2^{-k}\right)^{-1} \eta_{0}(\sqrt{\tau}+\xi)$ at the expense of an error dominated by

$$
C\left[2^{-k}+\left(2^{2 k}|\sqrt{\tau}+\xi|^{2}+1\right)^{-1}\right] \mathbf{1}_{[0, C]}(|\sqrt{\tau}+\xi|) .
$$

The $L_{\xi}^{1}$ norm of this error is $\leq C 2^{-k}$. Then we make the change of variables $\xi=-\sqrt{\tau}+\mu$ and use the uniform boundedness of the integral in $\mu$ in (4.20). The bound (4.21) follows.

\section{Linear EStimates}

For any $u \in C\left(\mathbb{R}: L^{2}\right)$ let $\widetilde{u}(., t) \in C\left(\mathbb{R}: L^{2}\right)$ denote its partial Fourier transform with respect to the variable $x$. For $\phi \in L^{2}(\mathbb{R})$ let $W(t) \phi \in C\left(\mathbb{R}: L^{2}\right)$ denote the solution of the free Benjamin-Ono evolution given by

$$
[W(t) \phi]^{\sim}(\xi, t)=e^{i t \omega(\xi)} \widehat{\phi}(\xi),
$$

where $\omega(\xi)$ is defined in (3.1). Assume $\psi: \mathbb{R} \rightarrow[0,1]$ is an even smooth function supported in the interval $[-8 / 5,8 / 5]$ and equal to 1 in the interval $[-5 / 4,5 / 4]$ and let $\varphi=\widehat{\psi}-\widehat{\psi}^{\prime \prime} \in \mathcal{S}(\mathbb{R})$.

Lemma 5.1. If $\sigma \geq 0$ and $\phi \in \widetilde{H}^{\sigma}$, then

$$
\|\psi(t) \cdot(W(t) \phi)\|_{F^{\sigma}} \leq C\|\phi\|_{\widetilde{H}^{\sigma}} .
$$


Proof of Lemma 5.1. A straightforward computation shows that

$$
\mathcal{F}[\psi(t) \cdot(W(t) \phi)](\xi, \tau)=\widehat{\phi}(\xi) \widehat{\psi}(\tau-\omega(\xi)) .
$$

Then, directly from the definitions,

$$
\begin{aligned}
& \|\psi(t) \cdot(W(t) \phi)\|_{F^{\sigma}}^{2}=\sum_{k \in \mathbb{Z}_{+}} 2^{2 \sigma k}\left\|\eta_{k}(\xi) \widehat{\phi}(\xi) \varphi(\tau-\omega(\xi))\right\|_{Z_{k}}^{2} \\
& \leq \sum_{k=1}^{\infty} 2^{2 \sigma k}\left\|\eta_{k}(\xi) \widehat{\phi}(\xi) \varphi(\tau-\omega(\xi))\right\|_{X_{k}}^{2}+\left\|\eta_{0}(\xi) \widehat{\phi}(\xi) \varphi(\tau-\omega(\xi))\right\|_{Z_{0}}^{2} .
\end{aligned}
$$

Since $\varphi \in \mathcal{S}(\mathbb{R})$, for any $k \geq 1$

$$
\left\|\eta_{k}(\xi) \widehat{\phi}(\xi) \varphi(\tau-\omega(\xi))\right\|_{X_{k}} \leq C\left\|\eta_{k} \cdot \widehat{\phi}\right\|_{L^{2}}
$$

For $k=0$, write $\eta_{0} \cdot \widehat{\phi}=g+\sum_{k^{\prime} \leq 1} h_{k^{\prime}}, h_{k^{\prime}}$ supported in $I_{k^{\prime}}$ and

$$
\left\|\mathcal{F}_{1}^{-1}(g)\right\|_{L_{x}^{1}}+\sum_{k^{\prime} \leq 1} 2^{-k^{\prime}}\left\|h_{k^{\prime}}\right\|_{L^{2}} \leq 2\left\|\eta_{0} \cdot \widehat{\phi}\right\|_{B_{0}} .
$$

Then

$$
\begin{aligned}
& \|g(\xi) \varphi(\tau-\omega(\xi))\|_{Z_{0}} \leq\|g(\xi) \varphi(\tau)\|_{Y_{0}}+\|g(\xi)[\varphi(\tau-\omega(\xi))-\varphi(\tau)]\|_{X_{0}} \\
& \leq C\left\|\mathcal{F}_{1}^{-1}(g)\right\|_{L_{x}^{1}}+C\left\|g(\xi) \xi^{2}(1+|\tau|)^{-4}\right\|_{X_{0}} \leq C\left\|\mathcal{F}_{1}^{-1}(g)\right\|_{L_{x}^{1}} .
\end{aligned}
$$

Also,

$$
\left\|h_{k^{\prime}}(\xi) \varphi(\tau-\omega(\xi))\right\|_{Z_{0}} \leq\left\|h_{k^{\prime}}(\xi) \varphi(\tau-\omega(\xi))\right\|_{X_{0}} \leq C 2^{-k^{\prime}}\left\|h_{k^{\prime}}\right\|_{L^{2}} .
$$

Lemma 5.1 follows from (5.4).

Lemma 5.2. If $\sigma \geq 0$ and $u \in N^{\sigma} \cap C\left(\mathbb{R}: H^{-2}\right)$, then

$$
\left\|\psi(t) \cdot \int_{0}^{t} W(t-s)(u(s)) d s\right\|_{F^{\sigma}} \leq C\|u\|_{N^{\sigma}} .
$$

Proof of Lemma 5.2, A straightforward computation shows that

$$
\mathcal{F}\left[\psi(t) \cdot \int_{0}^{t} W(t-s)(u(s)) d s\right](\xi, \tau)=c \int_{\mathbb{R}} \mathcal{F}(u)\left(\xi, \tau^{\prime}\right) \frac{\widehat{\psi}\left(\tau-\tau^{\prime}\right)-\widehat{\psi}(\tau-\omega(\xi))}{\tau^{\prime}-\omega(\xi)} d \tau^{\prime} .
$$

For $k \in \mathbb{Z}_{+}$let $f_{k}\left(\xi, \tau^{\prime}\right)=\mathcal{F}(u)\left(\xi, \tau^{\prime}\right) \eta_{k}(\xi) A_{k}\left(\xi, \tau^{\prime}\right)^{-1}$. For $f_{k} \in Z_{k}$ let

$$
T\left(f_{k}\right)(\xi, \tau)=\int_{\mathbb{R}} f_{k}\left(\xi, \tau^{\prime}\right) \frac{\varphi\left(\tau-\tau^{\prime}\right)-\varphi(\tau-\omega(\xi))}{\tau^{\prime}-\omega(\xi)} A_{k}\left(\xi, \tau^{\prime}\right) d \tau^{\prime} .
$$

In view of the definitions, it suffices to prove that

$$
\|T\|_{Z_{k} \rightarrow Z_{k}} \leq C \text { uniformly in } k \in \mathbb{Z}_{+} .
$$

We consider first the case $k \geq 1$. To prove (5.7), we use the representation (4.1). Assume first that $f_{k}=f_{k, j}$ is a function supported in $D_{k, j}$. Let $f_{k, j}^{\#}\left(\xi, \mu^{\prime}\right)=$ $f_{k, j}\left(\xi, \mu^{\prime}+\omega(\xi)\right)$ and $T\left(f_{k, j}\right) \#(\xi, \mu)=T\left(f_{k, j}\right)(\xi, \mu+\omega(\xi))$. Then,

$$
T\left(f_{k, j}\right)^{\#}(\xi, \mu)=\int_{\mathbb{R}} f_{k, j}^{\#}\left(\xi, \mu^{\prime}\right) \frac{\varphi\left(\mu-\mu^{\prime}\right)-\varphi(\mu)}{\mu^{\prime}}\left(\mu^{\prime}+i\right) d \mu^{\prime} .
$$

We use the elementary bound

$$
\left|\frac{\varphi\left(\mu-\mu^{\prime}\right)-\varphi(\mu)}{\mu^{\prime}}\left(\mu^{\prime}+i\right)\right| \leq C\left[(1+|\mu|)^{-4}+\left(1+\left|\mu-\mu^{\prime}\right|\right)^{-4}\right] .
$$


Then, using (5.8),

$$
\begin{aligned}
\left|T\left(f_{k, j}\right)^{\#}(\xi, \mu)\right| & \leq C(1+|\mu|)^{-4} 2^{j / 2}\left[\int_{\widetilde{I}_{j}}\left|f_{k, j}^{\#}\left(\xi, \mu^{\prime}\right)\right|^{2} d \mu^{\prime}\right]^{1 / 2} \\
& +C \eta_{[j-2, j+2]}(\mu) \int_{\widetilde{I}_{j}}\left|f_{k, j}^{\#}\left(\xi, \mu^{\prime}\right)\right|\left(1+\left|\mu-\mu^{\prime}\right|\right)^{-4} d \mu^{\prime} .
\end{aligned}
$$

It follows from the definition of the spaces $X_{k}$ that

$$
\|T\|_{X_{k} \rightarrow X_{k}} \leq C \text { uniformly in } k \geq 1,
$$

as desired.

Assume now that $f_{k}=g_{k} \in Y_{k}$, so $k \geq 100$. In view of Lemma 4.1(b), (c), and (5.9), we may assume that $g_{k}$ is supported in the set $\left\{\left(\xi, \tau^{\prime}\right):\left|\tau^{\prime}-\omega(\xi)\right| \leq 2^{k-20}\right\}$. We write

$$
g_{k}\left(\xi, \tau^{\prime}\right)=\frac{\tau^{\prime}-\omega(\xi)}{\tau^{\prime}-\omega(\xi)+i} g_{k}\left(\xi, \tau^{\prime}\right)+\frac{i}{\tau^{\prime}-\omega(\xi)+i} g_{k}\left(\xi, \tau^{\prime}\right) .
$$

Using Lemma 4.1(b), $\left\|i\left(\tau^{\prime}-\omega(\xi)+i\right)^{-1} g_{k}\left(\xi, \tau^{\prime}\right)\right\|_{X_{k}} \leq C\left\|g_{k}\right\|_{Y_{k}}$. In view of (5.9), it suffices to prove that

$$
\left\|\int_{\mathbb{R}} g_{k}\left(\xi, \tau^{\prime}\right) \varphi\left(\tau-\tau^{\prime}\right) d \tau^{\prime}\right\|_{Z_{k}}+\left\|\varphi(\tau-\omega(\xi)) \int_{\mathbb{R}} g_{k}\left(\xi, \tau^{\prime}\right) d \tau^{\prime}\right\|_{X_{k}} \leq C\left\|g_{k}\right\|_{Y_{k}}
$$

The bound for the second term in the left-hand side of (5.10) follows from (4.14) with $t=0$. To bound the first term, we write

$$
g_{k}\left(\xi, \tau^{\prime}\right)=g_{k}\left(\xi, \tau^{\prime}\right)\left[\frac{\tau^{\prime}-\omega(\xi)+i}{\tau-\omega(\xi)+i}+\frac{\tau-\tau^{\prime}}{\tau-\omega(\xi)+i}\right] .
$$

The first term in the left-hand side of (5.10) is dominated by

$$
\begin{aligned}
& C\left\|\eta_{[0, k-1]}(\tau-\omega(\xi))(\tau-\omega(\xi)+i)^{-1} \int_{\mathbb{R}} g_{k}\left(\xi, \tau^{\prime}\right)\left(\tau^{\prime}-\omega(\xi)+i\right) \varphi\left(\tau-\tau^{\prime}\right) d \tau^{\prime}\right\|_{Y_{k}} \\
& +C \sum_{j \leq k} 2^{j / 2}\left\|\eta_{j}(\tau-\omega(\xi))(\tau-\omega(\xi)+i)^{-1} \int_{\mathbb{R}} g_{k}\left(\xi, \tau^{\prime}\right) \varphi\left(\tau-\tau^{\prime}\right)\left(\tau-\tau^{\prime}\right) d \tau^{\prime}\right\|_{L^{2}} \\
& +C \sum_{j \geq k-1} 2^{j / 2} \beta_{k, j}\left\|\eta_{j}(\tau-\omega(\xi)) \int_{\mathbb{R}} g_{k}\left(\xi, \tau^{\prime}\right) \varphi\left(\tau-\tau^{\prime}\right) d \tau^{\prime}\right\| \|_{L^{2}} .
\end{aligned}
$$

For the first term in (5.11), we use Lemma 4.1(c) to bound it by

$$
C 2^{-k / 2}\left\|\mathcal{F}_{1}^{-1}(\varphi) \cdot \mathcal{F}^{-1}\left[\left(\tau^{\prime}-\omega(\xi)+i\right) g_{k}\left(\xi, \tau^{\prime}\right)\right]\right\|_{L_{x}^{1} L_{t}^{2}} \leq C\left\|g_{k}\right\|_{Y_{k}}
$$

as desired. Let $g_{k}^{\#}\left(\xi, \mu^{\prime}\right)=g_{k}\left(\xi, \mu^{\prime}+\omega(\xi)\right)$ and for $j^{\prime} \in[0, k-20]$ let $g_{k, j^{\prime}}^{\#}\left(\xi, \mu^{\prime}\right)=$ $g_{k}^{\#}\left(\xi, \mu^{\prime}\right) \eta_{j^{\prime}}\left(\mu^{\prime}\right)$. In view of Lemma 4.1(b), $2^{j^{\prime} / 2}\left\|g_{k, j^{\prime}}\right\|_{L^{2}} \leq C\left\|g_{k}\right\|_{Y_{k}}$, so the second term in (5.11) is dominated by

$$
C \sum_{j=0}^{k} \sum_{j^{\prime}=0}^{k-20} 2^{-j / 2} 2^{-j^{\prime} / 2}\left[2^{j^{\prime} / 2}\left\|g_{k, j^{\prime}}\right\|_{L^{2}}\right] \leq C\left\|g_{k}\right\|_{Y_{k}} .
$$


The third term in (5.11) is dominated by

$$
C \sum_{j=k-1}^{\infty} \sum_{j^{\prime}=0}^{k-20} 2^{-3 j} 2^{j^{\prime} / 2}\left\|g_{k, j^{\prime}}\right\|_{L^{2}} \leq C\left\|g_{k}\right\|_{Y_{k}},
$$

since $\varphi \in \mathcal{S}(\mathbb{R})$. This completes the proof of (5.10).

We consider now the case $k=0$. To prove (5.7), we use the representation (4.2). Assume first that $f_{0}=f_{0, j}^{k^{\prime}}$ is a function supported in $D_{k^{\prime}, j},\left\|f_{0}\right\|_{Z_{0}} \approx 2^{j-k^{\prime}}\left\|f_{0, j}^{k^{\prime}}\right\|_{L^{2}}$. For $|\xi| \leq 2$ we have the elementary bound

$$
\left|\frac{\varphi\left(\tau-\tau^{\prime}\right)-\varphi(\tau-\omega(\xi))}{\tau^{\prime}-\omega(\xi)}\left(\tau^{\prime}+i\right)\right| \leq C\left[(1+|\tau|)^{-4}+\left(1+\left|\tau-\tau^{\prime}\right|\right)^{-4}\right] .
$$

Then, using the formula (5.6),

$$
\begin{aligned}
\left|T\left(f_{0, j}^{k^{\prime}}\right)(\xi, \tau)\right| & \leq C(1+|\tau|)^{-4} 2^{j / 2}\left[\int_{\widetilde{I}_{j}}\left|f_{0, j}^{k^{\prime}}\left(\xi, \tau^{\prime}\right)\right|^{2} d \tau^{\prime}\right]^{1 / 2} \\
& +C \eta_{[j-4, j+4]}(\tau) \int_{\widetilde{I}_{j}}\left|f_{0, j}^{k^{\prime}}\left(\xi, \tau^{\prime}\right)\right|\left(1+\left|\tau-\tau^{\prime}\right|\right)^{-4} d \tau^{\prime}
\end{aligned}
$$

It follows from the definition of the spaces $X_{0}$ that $\|T\|_{X_{0} \rightarrow X_{0}} \leq C$, as desired.

Assume now that $f_{0}=g_{0, j}$ is supported in $\widetilde{I}_{0} \times \widetilde{I}_{j}$. We can write

$$
\left\{\begin{array}{l}
g_{0, j}\left(\xi, \tau^{\prime}\right)=2^{-j} \eta_{[0,1]}(\xi) \eta_{[j-1, j+1]}\left(\tau^{\prime}\right) \int_{\mathbb{R}} e^{-i x \xi} h\left(x, \tau^{\prime}\right) d x \\
2^{j}\left\|\mathcal{F}^{-1}\left(g_{0, j}\right)\right\|_{L_{x}^{1} L_{t}^{2}}=C\|h\|_{L_{x}^{1} L_{\tau^{\prime}}^{2}} .
\end{array}\right.
$$

We have two cases: $j \leq 5$ and $j \geq 6$. If $j \leq 5$, we write

$$
\frac{\varphi\left(\tau-\tau^{\prime}\right)-\varphi(\tau-\omega(\xi))}{\tau^{\prime}-\omega(\xi)}=c \int_{0}^{1} \varphi^{\prime}\left(\tau-\alpha \tau^{\prime}-(1-\alpha) \omega(\xi)\right) d \alpha .
$$

For (5.7), it suffices to prove that

$$
\left\|\int_{\mathbb{R}} g_{0, j}\left(\xi, \tau^{\prime}\right) \varphi^{\prime}\left(\tau-\alpha \tau^{\prime}-(1-\alpha) \omega(\xi)\right)\left(\tau^{\prime}+i\right) d \tau^{\prime}\right\|_{Z_{0}} \leq C\left\|\mathcal{F}^{-1}\left(g_{0, j}\right)\right\|_{L_{x}^{1} L_{t}^{2}}
$$

for any $\alpha \in[0,1]$. For $|\xi| \leq 2$ and $\left|\tau^{\prime}\right| \leq C$ we write

$$
\varphi^{\prime}\left(\tau-\alpha \tau^{\prime}-(1-\alpha) \omega(\xi)\right)\left(\tau^{\prime}+i\right)=\varphi^{\prime}\left(\tau-\alpha \tau^{\prime}\right)\left(\tau^{\prime}+i\right)+R\left(\xi, \tau, \tau^{\prime}\right),
$$

where

$$
\left|R\left(\xi, \tau, \tau^{\prime}\right)\right| \leq C \xi^{2}(1+|\tau|)^{-4}
$$

The left-hand side of (5.13) is dominated by

$$
\left\|\int_{\mathbb{R}} g_{0, j}\left(\xi, \tau^{\prime}\right) \varphi^{\prime}\left(\tau-\alpha \tau^{\prime}\right)\left(\tau^{\prime}+i\right) d \tau^{\prime}\right\|_{Y_{0}}+C\left\|\xi^{2}(1+|\tau|)^{-4} \int_{\widetilde{I}_{j}}\left|g_{0, j}\left(\xi, \tau^{\prime}\right)\right| d \tau^{\prime}\right\|_{X_{0}},
$$

which is easily seen to be dominated by $\left\|\mathcal{F}^{-1}\left(g_{0, j}\right)\right\|_{L_{x}^{1} L_{t}^{2}}$ (using the representation (5.12)). This completes the proof of (5.7) in the case $j \leq 5$.

Assume now that $j \geq 6$. Since $\left|\tau^{\prime}\right| \geq C$ and $|\xi| \leq 2$, we can write

$$
\frac{\varphi\left(\tau-\tau^{\prime}\right)-\varphi(\tau-\omega(\xi))}{\tau^{\prime}-\omega(\xi)}\left(\tau^{\prime}+i\right)=\frac{\varphi\left(\tau-\tau^{\prime}\right)-\varphi(\tau)}{\tau^{\prime}}\left(\tau^{\prime}+i\right)+R^{\prime}\left(\xi, \tau, \tau^{\prime}\right),
$$

where

$$
\left|R^{\prime}\left(\xi, \tau, \tau^{\prime}\right)\right| \leq C \xi^{2}\left[(1+|\tau|)^{-4}+\left(1+\left|\tau-\tau^{\prime}\right|\right)^{-4}\right]
$$


Using the representation (5.12) and the definitions, it follows as before that $\left\|\int_{\mathbb{R}} g_{0, j}\left(\xi, \tau^{\prime}\right) \frac{\varphi\left(\tau-\tau^{\prime}\right)-\varphi(\tau)}{\tau^{\prime}}\left(\tau^{\prime}+i\right) d \tau^{\prime}\right\|_{Y_{0}}+\left\|\int_{\mathbb{R}}\left|g_{0, j}\left(\xi, \tau^{\prime}\right)\right| \cdot\left|R^{\prime}\left(\xi, \tau, \tau^{\prime}\right)\right| d \tau^{\prime}\right\|_{X_{0}}$ is dominated by $C 2^{j}\left\|\mathcal{F}^{-1}\left(g_{0, j}\right)\right\|_{L_{x}^{1} L_{t}^{2}}$, which completes the proof of (5.7).

\section{Localized $L^{2}$ estimates}

In this section we prove several localized $L^{2}$ estimates for nonnegative functions. Such $L^{2}$ estimates are closely connected to bilinear estimates in the spaces $X^{\sigma, b}$ (see [21] for a more general discussion). For $\xi_{1}, \xi_{2} \in \mathbb{R}$ and $\omega: \mathbb{R} \rightarrow \mathbb{R}$ as in (3.1) let

$$
\Omega\left(\xi_{1}, \xi_{2}\right)=-\omega\left(\xi_{1}+\xi_{2}\right)+\omega\left(\xi_{1}\right)+\omega\left(\xi_{2}\right) .
$$

For compactly supported functions $f, g, h \in L^{2}(\mathbb{R} \times \mathbb{R})$ let

$$
J(f, g, h)=\int_{\mathbb{R}^{4}} f\left(\xi_{1}, \mu_{1}\right) g\left(\xi_{2}, \mu_{2}\right) h\left(\xi_{1}+\xi_{2}, \mu_{1}+\mu_{2}+\Omega\left(\xi_{1}, \xi_{2}\right)\right) d \xi_{1} d \xi_{2} d \mu_{1} d \mu_{2} .
$$

Given a triplet of real numbers $\left(\alpha_{1}, \alpha_{2}, \alpha_{3}\right)$, let $\min \left(\alpha_{1}, \alpha_{2}, \alpha_{3}\right)$, $\max \left(\alpha_{1}, \alpha_{2}, \alpha_{3}\right)$, and med $\left(\alpha_{1}, \alpha_{2}, \alpha_{3}\right)$ denote the minimum, the maximum, and the median (i.e., $\left.\operatorname{med}\left(\alpha_{1}, \alpha_{2}, \alpha_{3}\right)=\alpha_{1}+\alpha_{2}+\alpha_{3}-\max \left(\alpha_{1}, \alpha_{2}, \alpha_{3}\right)-\min \left(\alpha_{1}, \alpha_{2}, \alpha_{3}\right)\right)$ of the numbers $\alpha_{1}, \alpha_{2}$, and $\alpha_{3}$.

Lemma 6.1. Assume $k_{1}, k_{2}, k_{3} \in \mathbb{Z}, j_{1}, j_{2}, j_{3} \in \mathbb{Z}_{+}$, and $f_{k_{i}, j_{i}} \in L^{2}(\mathbb{R} \times \mathbb{R})$ are functions supported in $I_{k_{i}} \times \widetilde{I}_{j_{i}}, i=1,2,3$.

(a) For any $k_{1}, k_{2}, k_{3} \in \mathbb{Z}$ and $j_{1}, j_{2}, j_{3} \in \mathbb{Z}_{+}$,

$$
\left|J\left(f_{k_{1}, j_{1}}, f_{k_{2}, j_{2}}, f_{k_{3}, j_{3}}\right)\right| \leq C 2^{\min \left(k_{1}, k_{2}, k_{3}\right) / 2} 2^{\min \left(j_{1}, j_{2}, j_{3}\right) / 2} \prod_{i=1}^{3}\left\|f_{k_{i}, j_{i}}\right\|_{L^{2}} .
$$

(b) If $\max \left(k_{1}, k_{2}, k_{3}\right) \geq \min \left(k_{1}, k_{2}, k_{3}\right)+5$ and $i \in\{1,2,3\}$, then

$$
\left|J\left(f_{k_{1}, j_{1}}, f_{k_{2}, j_{2}}, f_{k_{3}, j_{3}}\right)\right| \leq C 2^{\left(j_{1}+j_{2}+j_{3}\right) / 2} 2^{-\left(j_{i}+k_{i}\right) / 2} \prod_{i=1}^{3}\left\|f_{k_{i}, j_{i}}\right\|_{L^{2}} .
$$

(c) For any $k_{1}, k_{2}, k_{3} \in \mathbb{Z}$ and $j_{1}, j_{2}, j_{3} \in \mathbb{Z}_{+}$,

$$
\left|J\left(f_{k_{1}, j_{1}}, f_{k_{2}, j_{2}}, f_{k_{3}, j_{3}}\right)\right| \leq C 2^{\min \left(j_{1}, j_{2}, j_{3}\right) / 2+\operatorname{med}\left(j_{1}, j_{2}, j_{3}\right) / 4} \prod_{i=1}^{3}\left\|f_{k_{i}, j_{i}}\right\|_{L^{2}} .
$$

Proof of Lemma 6.1, Let $A_{k_{i}}(\xi)=\left[\int_{\mathbb{R}}\left|f_{k_{i}, j_{i}}(\xi, \mu)\right|^{2} d \mu\right]^{1 / 2}, i=1,2,3$. Using the Hölder inequality and the support properties of the functions $f_{k_{i}, j_{i}}$,

$$
\begin{aligned}
\left|J\left(f_{k_{1}, j_{1}}, f_{k_{2}, j_{2}}, f_{k_{3}, j_{3}}\right)\right| & \leq C 2^{\min \left(j_{1}, j_{2}, j_{3}\right) / 2} \int_{\mathbb{R}^{2}} A_{k_{1}}\left(\xi_{1}\right) A_{k_{2}}\left(\xi_{2}\right) A_{k_{3}}\left(\xi_{1}+\xi_{2}\right) d \xi_{1} d \xi_{2} \\
& \leq C 2^{\min \left(k_{1}, k_{2}, k_{3}\right) / 2} 2^{\min \left(j_{1}, j_{2}, j_{3}\right) / 2} \prod_{i=1}^{3}\left\|f_{k_{i}, j_{i}}\right\|_{L^{2}}
\end{aligned}
$$

which is part (a).

For part (b) we observe that

$$
\left|\Omega\left(\xi_{1}, \xi_{2}\right)\right|=2 \min \left(\left|\xi_{1}\right|,\left|\xi_{2}\right|,\left|\xi_{1}+\xi_{2}\right|\right) \cdot \operatorname{med}\left(\left|\xi_{1}\right|,\left|\xi_{2}\right|,\left|\xi_{1}+\xi_{2}\right|\right) .
$$


Also, by examining the supports of the functions, $J\left(f_{k_{1}, j_{1}}, f_{k_{2}, j_{2}}, f_{k_{3}, j_{3}}\right) \equiv 0$ unless

$$
\max \left(k_{1}, k_{2}, k_{3}\right) \leq \operatorname{med}\left(k_{1}, k_{2}, k_{3}\right)+2,
$$

and

$$
\left\{\begin{array}{l}
\max \left(j_{1}, j_{2}, j_{3}\right) \in[\widetilde{k}-5, \widetilde{k}+5] \text { or } \\
\max \left(j_{1}, j_{2}, j_{3}\right) \geq \widetilde{k}+5 \text { and } \max \left(j_{1}, j_{2}, j_{3}\right)-\operatorname{med}\left(j_{1}, j_{2}, j_{3}\right) \leq 5,
\end{array}\right.
$$

where $\widetilde{k}=\min \left(k_{1}, k_{2}, k_{3}\right)+\operatorname{med}\left(k_{1}, k_{2}, k_{3}\right)$.

Simple changes of variables and the observation that the function $\omega$ is odd show that

$$
|J(f, g, h)|=|J(g, f, h)| \text { and }|J(f, g, h)|=|J(\tilde{f}, h, g)|,
$$

where $\tilde{f}(\xi, \mu)=f(-\xi,-\mu)$. Thus, by symmetry, in proving (6.4), we may assume $i=3$. Let

$B_{k_{3}}(\xi, \mu)=\left[\frac{1}{2^{j_{1}} 2^{j_{2}}} \int_{\mathbb{R}^{2}}\left|f_{k_{3}, j_{3}}(\xi, \mu+\alpha+\beta)\right|^{2}\left(1+\alpha / 2^{j_{1}}\right)^{-2}\left(1+\beta / 2^{j_{2}}\right)^{-2} d \alpha d \beta\right]^{1 / 2}$.

Clearly,

$$
\left\|B_{k_{3}}\right\|_{L^{2}}=C\left\|f_{k_{3}, j_{3}}\right\|_{L^{2}} \text { and } B_{k_{3}} \text { is supported in } I_{k_{3}} \times \mathbb{R} \text {. }
$$

Also, by the Hölder inequality,

$$
\begin{aligned}
& \left|J\left(f_{k_{1}, j_{1}}, f_{k_{2}, j_{2}}, f_{k_{3}, j_{3}}\right)\right| \\
& \quad \leq C 2^{\left(j_{1}+j_{2}\right) / 2} \int_{\mathbb{R}^{2}} A_{k_{1}}\left(\xi_{1}\right) A_{k_{2}}\left(\xi_{2}\right) B_{k_{3}}\left(\xi_{1}+\xi_{2}, \Omega\left(\xi_{1}, \xi_{2}\right)\right) d \xi_{1} d \xi_{2} .
\end{aligned}
$$

We have three cases depending on the relative sizes of $\left|\xi_{1}\right|,\left|\xi_{2}\right|$ and $\left|\xi_{1}+\xi_{2}\right|$. Let

$$
\left\{\begin{array}{l}
R_{1}=\left\{\left(\xi_{1}, \xi_{2}\right):\left|\xi_{1}+\xi_{2}\right| \leq\left|\xi_{1}\right| \text { and }\left|\xi_{2}\right| \leq\left|\xi_{1}\right|\right\}, \\
R_{2}=\left\{\left(\xi_{1}, \xi_{2}\right):\left|\xi_{1}+\xi_{2}\right| \leq\left|\xi_{2}\right| \text { and }\left|\xi_{1}\right| \leq\left|\xi_{2}\right|\right\}, \\
R_{3}=\left\{\left(\xi_{1}, \xi_{2}\right):\left|\xi_{1}\right| \leq\left|\xi_{1}+\xi_{2}\right| \text { and }\left|\xi_{2}\right| \leq\left|\xi_{1}+\xi_{2}\right|\right\} .
\end{array}\right.
$$

For $\left(\xi_{1}, \xi_{2}\right) \in R_{1}$, using (6.7), $\Omega\left(\xi_{1}, \xi_{2}\right)= \pm 2 \xi_{2}\left(\xi_{1}+\xi_{2}\right)$. We define $B_{k_{3}}^{\prime}(\xi, \mu)=$ $B_{k_{3}}(\xi, 2 \xi \mu),\left\|B_{k_{3}}^{\prime}\right\|_{L^{2}} \approx 2^{-k_{3} / 2}\left\|B_{k_{3}}\right\|_{L^{2}}$. The integral over $R_{1}$ in the right-hand side of (6.12) is dominated by

$$
\begin{gathered}
C \int_{\mathbb{R}^{2}} A_{k_{1}}\left(\xi_{1}\right) A_{k_{2}}\left(\xi_{2}\right)\left[B_{k_{3}}^{\prime}\left(\xi_{1}+\xi_{2}, \xi_{2}\right)+B_{k_{3}}^{\prime}\left(\xi_{1}+\xi_{2},-\xi_{2}\right)\right] d \xi_{1} d \xi_{2} \\
\leq C 2^{-k_{3} / 2}\left\|A_{k_{1}}\right\|_{L^{2}}\left\|A_{k_{2}}\right\|_{L^{2}}\left\|B_{k_{3}}\right\|_{L^{2}}
\end{gathered}
$$

which gives (6.4) in this case (see (6.11)).

The bound for the integral over $\left(\xi_{1}, \xi_{2}\right) \in R_{2}$ is identical. We consider now the integral over $\left(\xi_{1}, \xi_{2}\right) \in R_{3}$, in which case $\Omega\left(\xi_{1}, \xi_{2}\right)= \pm 2 \xi_{1} \xi_{2}$. By symmetry, to bound the right-hand side of (6.12), it suffices to bound

$$
\int_{R_{3}} A_{k_{1}}\left(\xi_{1}\right) A_{k_{2}}\left(\xi_{2}\right) B_{k_{3}}\left(\xi_{1}+\xi_{2}, 2 \xi_{1} \xi_{2}\right) d \xi_{1} d \xi_{2} .
$$

We define $B_{k_{3}}^{\prime \prime}(\xi, \mu)=B_{k_{3}}\left(\xi, \mu+\xi^{2} / 2\right)$, so $\left\|B_{k_{3}}^{\prime \prime}\right\|_{L^{2}}=\left\|B_{k_{3}}\right\|_{L^{2}}$. Using (6.8) and the assumption $\max \left(k_{1}, k_{2}, k_{3}\right) \geq \min \left(k_{1}, k_{2}, k_{3}\right)+5$, if $\xi_{1} \in I_{k_{1}}, \xi_{2} \in I_{k_{2}},\left(\xi_{1}, \xi_{2}\right) \in R_{3}$, and $\xi_{1}+\xi_{2} \in I_{k_{3}}$, then $\left|\xi_{1}-\xi_{2}\right| \geq 2^{k_{3}-100}$. The integral in (6.14) is dominated by

$$
\int_{\left\{\left|\xi_{1}-\xi_{2}\right| \geq 2^{k_{3}-100}\right\}} A_{k_{1}}\left(\xi_{1}\right) A_{k_{2}}\left(\xi_{2}\right) B_{k_{3}}^{\prime \prime}\left(\xi_{1}+\xi_{2},-\left(\xi_{1}-\xi_{2}\right)^{2} / 2\right) d \xi_{1} d \xi_{2}
$$


Using the Hölder inequality and a simple change of variables, the integral in (6.15) is dominated by $C 2^{-k_{3} / 2}\left\|A_{k_{1}}\right\|_{L^{2}}\left\|A_{k_{2}}\right\|_{L^{2}}\left\|B_{k_{3}}^{\prime \prime}\right\|_{L^{2}}$, which completes the proof of (6.4).

For part (c), using part (a), we may assume

$$
\operatorname{med}\left(j_{1}, j_{2}, j_{3}\right) \leq 2 \min \left(k_{1}, k_{2}, k_{3}\right) .
$$

Using (6.10), we may also assume $j_{1}=\min \left(j_{1}, j_{2}, j_{3}\right)$ and $j_{2}=\operatorname{med}\left(j_{1}, j_{2}, j_{3}\right)$. Let

$$
\widetilde{R}_{j_{2}}=\left\{\left(\xi_{1}, \xi_{2}\right):\left|\xi_{1}-\xi_{2}\right| \geq 2^{j_{2} / 2}\right\} .
$$

For the integral over $\left(\xi_{1}, \xi_{2}\right) \in{ }^{c} \widetilde{R}_{j_{2}}=\mathbb{R}^{2} \backslash \widetilde{R}_{j_{2}}$ we use a bound similar to (6.6):

$$
\begin{aligned}
\mid \int_{\widetilde{R}_{j_{2}} \times \mathbb{R}^{2}} & f_{k_{1}, j_{1}}\left(\xi_{1}, \mu_{1}\right) f_{k_{2}, j_{2}}\left(\xi_{2}, \mu_{2}\right) f_{k_{3}, j_{3}}\left(\xi_{1}+\xi_{2}, \mu_{1}+\mu_{2}+\Omega\left(\xi_{1}, \xi_{2}\right)\right) d \xi_{1} d \xi_{2} d \mu_{1} d \mu_{2} \mid \\
& \leq C 2^{j_{1} / 2} \int_{\widetilde{R}_{j_{2}}} A_{k_{1}}\left(\xi_{1}\right) A_{k_{2}}\left(\xi_{2}\right) A_{k_{3}}\left(\xi_{1}+\xi_{2}\right) d \xi_{1} d \xi_{2} \\
& \leq C 2^{j_{1} / 2} \iint_{|\mu| \leq 2^{j_{2} / 2}} A_{k_{1}}\left(\xi_{2}+\mu\right) A_{k_{2}}\left(\xi_{2}\right) A_{k_{3}}\left(2 \xi_{2}+\mu\right) d \xi_{2} d \mu \\
& \leq C 2^{j_{1} / 2} \int_{|\mu| \leq 2^{j_{2} / 2}}\left(\int_{\mathbb{R}}\left|A_{k_{1}}\left(\xi_{2}+\mu\right)\right|^{2}\left|A_{k_{2}}\left(\xi_{2}\right)\right|^{2} d \xi_{2}\right)^{1 / 2} \|\left. A_{k_{3}}\right|_{L^{2}} d \mu \\
& \leq C 2^{j_{1} / 2} 2^{j_{2} / 4}|| A_{k_{1}}\left\|_{L^{2}}|| A_{k_{2}}\right\|_{L^{2}} \|\left. A_{k_{3}}\right|_{L^{2}},
\end{aligned}
$$

which suffices for (6.5). For the integral over $\left(\xi_{1}, \xi_{2}\right) \in \widetilde{R}_{j_{2}}$ we use a bound similar to (6.12):

$$
\begin{aligned}
& \left|\int_{\widetilde{R}_{j_{2}} \times \mathbb{R}^{2}} f_{k_{1}, j_{1}}\left(\xi_{1}, \mu_{1}\right) f_{k_{2}, j_{2}}\left(\xi_{2}, \mu_{2}\right) f_{k_{3}, j_{3}}\left(\xi_{1}+\xi_{2}, \mu_{1}+\mu_{2}+\Omega\left(\xi_{1}, \xi_{2}\right)\right) d \xi_{1} d \xi_{2} d \mu_{1} d \mu_{2}\right| \\
& \quad \leq C 2^{\left(j_{1}+j_{2}\right) / 2} \int_{\widetilde{R}_{j_{2}}} A_{k_{1}}\left(\xi_{1}\right) A_{k_{2}}\left(\xi_{2}\right) B_{k_{3}}\left(\xi_{1}+\xi_{2}, \Omega\left(\xi_{1}, \xi_{2}\right)\right) d \xi_{1} d \xi_{2} .
\end{aligned}
$$

We further decompose the integral in the right-hand side of (6.17) into three parts, corresponding to the regions $R_{1}, R_{2}$, and $R_{3}$. Using (6.13), the integrals over the regions $\widetilde{R}_{j_{2}} \cap R_{1}$ and $\widetilde{R}_{j_{2}} \cap R_{2}$ are dominated by $C 2^{-k_{3} / 2}\left\|A_{k_{1}}\right\|_{L^{2}}\left\|A_{k_{2}}\right\|_{L^{2}}\left\|B_{k_{3}}\right\|_{L^{2}}$, which suffices in view of the assumption (6.16). For the integral over the region $\widetilde{R}_{j_{2}} \cap R_{3}$, by symmetry it suffices to control

$$
\int_{\widetilde{R}_{j_{2}} \cap R_{3}} A_{k_{1}}\left(\xi_{1}\right) A_{k_{2}}\left(\xi_{2}\right) B_{k_{3}}\left(\xi_{1}+\xi_{2}, 2 \xi_{1} \xi_{2}\right) d \xi_{1} d \xi_{2} .
$$

As in the estimate of the integral in (6.14), the integral in (6.18) is dominated by

$$
\int_{\left\{\left|\xi_{1}-\xi_{2}\right| \geq 2^{j_{2} / 2}\right\}} A_{k_{1}}\left(\xi_{1}\right) A_{k_{2}}\left(\xi_{2}\right) B_{k_{3}}^{\prime \prime}\left(\xi_{1}+\xi_{2},-\left(\xi_{1}-\xi_{2}\right)^{2} / 2\right) d \xi_{1} d \xi_{2} .
$$

The bound (6.5) follows using the Hölder inequality and a simple change of variables.

We restate now Lemma 6.1 in a form that is suitable for the bilinear estimates in the next sections. 
Corollary 6.2. Assume $k_{1}, k_{2}, k_{3} \in \mathbb{Z}, j_{1}, j_{2}, j_{3} \in \mathbb{Z}_{+}$, and $f_{k_{i}, j_{i}} \in L^{2}(\mathbb{R} \times \mathbb{R})$ are functions supported in $D_{k_{i}, j_{i}}, i=1,2$.

(a) For any $k_{1}, k_{2}, k_{3} \in \mathbb{Z}$ and $j_{1}, j_{2}, j_{3} \in \mathbb{Z}_{+}$,

$$
\left\|\mathbf{1}_{D_{k_{3}, j_{3}}}(\xi, \tau)\left(f_{k_{1}, j_{1}} * f_{k_{2}, j_{2}}\right)(\xi, \tau)\right\|_{L^{2}} \leq C 2^{\min \left(k_{1}, k_{2}, k_{3}\right) / 2} 2^{\min \left(j_{1}, j_{2}, j_{3}\right) / 2} \prod_{i=1}^{2}\left\|f_{k_{i}, j_{i}}\right\|_{L^{2}} .
$$

(b) If $\max \left(k_{1}, k_{2}, k_{3}\right) \geq \min \left(k_{1}, k_{2}, k_{3}\right)+5$ and $i \in\{1,2,3\}$, then

$$
\left\|\mathbf{1}_{D_{k_{3}, j_{3}}}(\xi, \tau)\left(f_{k_{1}, j_{1}} * f_{k_{2}, j_{2}}\right)(\xi, \tau)\right\|_{L^{2}} \leq C 2^{\left(j_{1}+j_{2}+j_{3}\right) / 2} 2^{-\left(j_{i}+k_{i}\right) / 2} \prod_{i=1}^{2}\left\|f_{k_{i}, j_{i}}\right\|_{L^{2}} .
$$

(c) For any $k_{1}, k_{2}, k_{3} \in \mathbb{Z}$ and $j_{1}, j_{2}, j_{3} \in \mathbb{Z}_{+}$,

$$
\left\|\mathbf{1}_{D_{k_{3}, j_{3}}}(\xi, \tau)\left(f_{k_{1}, j_{1}} * f_{k_{2}, j_{2}}\right)(\xi, \tau)\right\|_{L^{2}} \leq C 2^{\min \left(j_{1}, j_{2}, j_{3}\right) / 2+\operatorname{med}\left(j_{1}, j_{2}, j_{3}\right) / 4} \prod_{i=1}^{2}\left\|f_{k_{i}, j_{i}}\right\|_{L^{2}} .
$$

(d) In addition, $\mathbf{1}_{D_{k_{3}, j_{3}}}(\xi, \tau)\left(f_{k_{1}, j_{1}} * f_{k_{2}, j_{2}}\right)(\xi, \tau) \equiv 0$ unless

$$
\max \left(k_{1}, k_{2}, k_{3}\right) \leq \operatorname{med}\left(k_{1}, k_{2}, k_{3}\right)+2 \text {, }
$$

and

$$
\left\{\begin{array}{l}
\max \left(j_{1}, j_{2}, j_{3}\right) \in[\widetilde{k}-8, \widetilde{k}+8] \text { or } \\
\max \left(j_{1}, j_{2}, j_{3}\right) \geq \widetilde{k}+8 \text { and } \max \left(j_{1}, j_{2}, j_{3}\right)-\operatorname{med}\left(j_{1}, j_{2}, j_{3}\right) \leq 10
\end{array}\right.
$$

where $\widetilde{k}=\min \left(k_{1}, k_{2}, k_{3}\right)+\operatorname{med}\left(k_{1}, k_{2}, k_{3}\right)$.

Proof of Corollary 6.2. Clearly,

$$
\left\|\mathbf{1}_{D_{k_{3}, j_{3}}}(\xi, \tau)\left(f_{k_{1}, j_{1}} * f_{k_{2}, j_{2}}\right)(\xi, \tau)\right\|_{L^{2}}=\sup _{\|f\|_{L^{2}}=1}\left|\int_{D_{k_{3}, j_{3}}} f \cdot\left(f_{k_{1}, j_{1}} * f_{k_{2}, j_{2}}\right) d \xi d \tau\right| .
$$

Let $f_{k_{3}, j_{3}}=\mathbf{1}_{D_{k_{3}, j_{3}}} \cdot f$, and then $f_{k_{i}, j_{i}}^{\#}(\xi, \mu)=f_{k_{i}, j_{i}}(\xi, \mu+\omega(\xi)), i=1,2,3$. The functions $f_{k_{i}, j_{i}}^{\#}$ are supported in $I_{k_{i}} \times \bigcup_{|m| \leq 3} \widetilde{I}_{j_{i}+m},\left\|f_{k_{i}, j_{i}}^{\#}\right\|_{L^{2}}=\left\|f_{k_{i}, j_{i}}\right\|_{L^{2}}$, and, using simple changes of variables,

$$
\int_{D_{k_{3}, j_{3}}} f \cdot\left(f_{k_{1}, j_{1}} * f_{k_{2}, j_{2}}\right) d \xi d \tau=J\left(f_{k_{1}, j_{1}}^{\#}, f_{k_{2}, j_{2}}^{\#}, f_{k_{3}, j_{3}}^{\#}\right) .
$$

Corollary 6.2 follows from Lemma 6.1, (6.8), and (6.9).

\section{Bilinear estimates I}

In this section we prove two bilinear estimates, which correspond to Low $\times$ High $\rightarrow$ High interactions:

Proposition 7.1. Assume $k \geq 20, k_{2} \in[k-2, k+2], f_{k_{2}} \in Z_{k_{2}}$, and $f_{0} \in Z_{0}$. Then

$$
2^{k}\left\|\eta_{k}(\xi) \cdot(\tau-\omega(\xi)+i)^{-1} f_{k_{2}} * f_{0}\right\|_{Z_{k}} \leq C\left\|f_{k_{2}}\right\|\left\|_{Z_{k_{2}}}\right\| f_{0} \|_{Z_{0}}
$$


Proposition 7.2. Assume $k \geq 20, k_{2} \in[k-2, k+2], f_{k_{2}} \in Z_{k_{2}}$, and $f_{k_{1}} \in Z_{k_{1}}$ for any $k_{1} \in[1, k-10] \cap \mathbb{Z}$. Then

$$
2^{k}\left\|\eta_{k}(\xi)(\tau-\omega(\xi)+i)^{-1} f_{k_{2}} * \sum_{k_{1}=1}^{k-10} f_{k_{1}}\right\|_{Z_{k}} \leq C\left\|f_{k_{2}}\right\|_{Z_{k_{2}}} \sup _{k_{1} \in[1, k-10]}\left\|\left(I-\partial_{\tau}^{2}\right) f_{k_{1}}\right\|_{Z_{k_{1}}} .
$$

The main ingredients in the proofs of Propositions 7.1 and 7.2 are the definitions, the representations (4.1), (4.2), and (4.8), Lemma 4.1, Lemma 4.2(b), (c), Corollary 6.2, and the $L^{2}$ estimates in Lemma 7.3 below.

Lemma 7.3. Assume that $k \geq 20, k_{1} \in(-\infty, k-10] \cap \mathbb{Z}, k_{2} \in[k-2, k+2]$, $j, j_{1}, j_{2} \in \mathbb{Z}_{+}, f_{k_{1}, j_{1}}$ is an $L^{2}$ function supported in $D_{k_{1}, j_{1}}$, and $f_{k_{2}, j_{2}}$ is an $L^{2}$ function supported in $D_{k_{2}, j_{2}}$. Then, with $\gamma_{k, k_{1}}=\left(2^{k_{1} / 2}+2^{-k / 2}\right)^{-1}$,

$$
\begin{aligned}
2^{k} 2^{j / 2} \beta_{k, j} \| \eta_{k}(\xi) & \eta_{j}(\tau-\omega(\xi))(\tau-\omega(\xi)+i)^{-1}\left(f_{k_{1}, j_{1}} * f_{k_{2}, j_{2}}\right) \|_{L^{2}} \\
& \leq C \gamma_{k, k_{1}} \cdot 2^{j_{1} / 2} \beta_{k_{1}, j_{1}}\left\|f_{k_{1}, j_{1}}\right\|_{L^{2}} \cdot 2^{j_{2} / 2} \beta_{k_{2}, j_{2}}|| f_{k_{2}, j_{2}} \|_{L^{2}},
\end{aligned}
$$

where, by definition, $\beta_{k_{1}, j_{1}}=2^{j_{1} / 2}$ if $k_{1} \leq 0$. In addition,

$$
\mathbf{1}_{D_{k, j}}(\xi, \tau)\left(f_{k_{1}, j_{1}} * f_{k_{2}, j_{2}}\right) \equiv 0
$$

unless

$$
\left\{\begin{array}{l}
\max \left(j, j_{1}, j_{2}\right) \in\left[k+k_{1}-10, k+k_{1}+10\right] \text { or } \\
\max \left(j, j_{1}, j_{2}\right) \geq k+k_{1}+10 \text { and } \max \left(j, j_{1}, j_{2}\right)-\operatorname{med}\left(j, j_{1}, j_{2}\right) \leq 10 .
\end{array}\right.
$$

Remark. The bound (17.3) holds for $k_{1}$ both positive and negative. However, when $k_{1} \leq 0$, the right-hand side contains the large factor $\gamma_{k, k_{1}}$. This factor is the main reason why interactions between "general" $L^{2}$ functions of very low frequency and derivatives of $L^{2}$ functions of high frequency cannot be estimated using our bilinear estimates.

Proof of Lemma 7.3. The restriction (7.4) follows directly from (6.23). For (7.3) we use the bounds (6.19), (6.20), and (6.21) in Corollary 6.2, The left-hand side of (7.3) is dominated by

$$
2^{k} 2^{-j / 2} \beta_{k, j}\left\|\mathbf{1}_{D_{k, j}}(\xi, \tau)\left(f_{k_{1}, j_{1}} * f_{k_{2}, j_{2}}\right)\right\|_{L^{2}} .
$$

For (7.3) it suffices to prove that

$$
\begin{aligned}
& \left\|\mathbf{1}_{D_{k, j}}(\xi, \tau)\left(f_{k_{1}, j_{1}} * f_{k_{2}, j_{2}}\right)\right\|_{L^{2}} \\
& \quad \leq C 2^{-k} \gamma_{k, k_{1}} 2^{\left(j+j_{1}+j_{2}\right) / 2} \beta_{k_{1}, j_{1}} \beta_{k_{2}, j_{2}} \beta_{k, j}^{-1}|| f_{k_{1}, j_{1}}\left\|_{L^{2}}\right\| f_{k_{2}, j_{2}} \|_{L^{2}} .
\end{aligned}
$$

Let $\Pi=\left\|f_{k_{1}, j_{1}}\right\|_{L^{2}}\left\|f_{k_{2}, j_{2}}\right\|_{L^{2}}$. We have several cases: if $j=\max \left(j, j_{1}, j_{2}\right)$, then, using (6.20), the left-hand side of (7.5) is dominated by $C 2^{-k / 2} 2^{\left(j_{1}+j_{2}\right) / 2} \Pi$; in addition $\beta_{k_{1}, j_{1}} \beta_{k_{2}, j_{2}} \beta_{k, j}^{-1} \geq C^{-1}$ and $2^{j / 2} \geq C^{-1}\left(2^{\left(k+k_{1}\right) / 2}+1\right)$, using (7.4), so the bound (7.5) follows in this case.

If $j_{2}=\max \left(j, j_{1}, j_{2}\right)$, then, using (6.20), the left-hand side of (17.5) is dominated by $C 2^{-k / 2} 2^{\left(j+j_{1}\right) / 2} \Pi$; in addition

$$
\beta_{k_{1}, j_{1}} \beta_{k_{2}, j_{2}} \beta_{k, j}^{-1} \geq C^{-1} \text { and } 2^{j_{2} / 2} \geq C^{-1}\left(2^{\left(k+k_{1}\right) / 2}+1\right),
$$

using (7.4), so the bound (7.5) follows in this case. 
If $j_{1}=\max \left(j, j_{1}, j_{2}\right) \geq k+k_{1}-20$ and $k_{1} \geq 0$, then, using (6.20) and (6.21), the left-hand side of (7.5) is dominated by

$$
C 2^{-j_{1} / 2}\left(2^{k_{1} / 2}+2^{\max \left(j, j_{2}\right) / 4}\right)^{-1} 2^{\left(j+j_{1}+j_{2}\right) / 2} \Pi
$$

in addition $2^{j_{1} / 2} \beta_{k_{1}, j_{1}} \geq C^{-1} 2^{j_{1}-k_{1}}, \beta_{k_{2}, j_{2}} \geq 1$, and $\beta_{k, j} \leq C \beta_{k, j_{1}}$. Using (7.4), $2^{j_{1}} \beta_{k, j_{1}}^{-1} \geq C^{-1} 2^{k+k_{1}}$, and the bound (7.5) follows. We notice also that the restriction $j_{1}=\max \left(j, j_{1}, j_{2}\right)$ was not important. For later use, we restate the stronger estimate that we obtain in this case: if $k_{1} \geq 0$ and $j_{1} \geq k+k_{1}-20$, then

$$
\begin{aligned}
& 2^{k} 2^{j / 2} \beta_{k, j}\left\|\eta_{k}(\xi) \eta_{j}(\tau-\omega(\xi))(\tau-\omega(\xi)+i)^{-1}\left(f_{k_{1}, j_{1}} * f_{k_{2}, j_{2}}\right)\right\|_{L^{2}} \\
& \quad \leq C\left(2^{k_{1} / 2}+2^{\max \left(j, j_{2}\right) / 4}\right)^{-1} \cdot 2^{j_{1} / 2} \beta_{k_{1}, j_{1}}\left\|f_{k_{1}, j_{1}}\right\|_{L^{2}} \cdot 2^{j_{2} / 2} \beta_{k_{2}, j_{2}}\left\|f_{k_{2}, j_{2}}\right\|_{L^{2}} .
\end{aligned}
$$

If $j_{1}=\max \left(j, j_{1}, j_{2}\right) \geq k+k_{1}-20$ and $k_{1} \leq 1$, then, using (6.19), the left-hand side of (7.5) is dominated by $C 2^{k_{1} / 2} 2^{\left(j+j_{2}\right) / 2} 2^{-\max \left(j, j_{2}\right) / 2} \Pi$; in addition $2^{j_{1} / 2} \beta_{k_{1}, j_{1}}$ $=2^{j_{1}}, \beta_{k_{2}, j_{2}} \geq 1$, and $\beta_{k, j} \leq C \beta_{k, j_{1}}$. Using (17.4), $2^{j_{1}} \beta_{k, j_{1}}^{-1} \geq C^{-1}\left(2^{k+k_{1}}+1\right)$, and the bound (7.5) follows since $2^{k_{1}}+2^{-k} \geq C^{-1} \gamma_{k, k_{1}}^{-2}$. For later use, we restate the stronger estimate that we obtain in this last case: if $k_{1} \leq 1$ and $j_{1} \geq k+k_{1}-20$, then

$$
\begin{aligned}
& 2^{k} 2^{j / 2} \beta_{k, j}\left\|\eta_{k}(\xi) \eta_{j}(\tau-\omega(\xi))(\tau-\omega(\xi)+i)^{-1}\left(f_{k_{1}, j_{1}} * f_{k_{2}, j_{2}}\right)\right\|_{L^{2}} \\
& \quad \leq C 2^{-\max \left(j, j_{2}\right) / 2} \gamma_{k, k_{1}} \cdot 2^{j_{1}}\left\|f_{k_{1}, j_{1}}\right\|_{L^{2}} \cdot 2^{j_{2} / 2} \beta_{k_{2}, j_{2}}\left\|f_{k_{2}, j_{2}}\right\|_{L^{2}}
\end{aligned}
$$

We prove now Propositions 7.1 and 7.2

Proof of Proposition 7.1. We use the representations (4.1) and (4.2) and analyze three cases.

Case 1: $f_{0}=f_{0, j_{1}}^{k_{1}}$ is supported in $D_{k_{1}, j_{1}}, f_{k_{2}}=f_{k_{2}, j_{2}}$ is supported in $D_{k_{2}, j_{2}}$, $j_{1}, j_{2} \geq 0, k_{1} \leq 1,\left\|f_{0}\right\|_{Z_{0}} \approx 2^{j_{1}-k_{1}}\left\|f_{0, j_{1}}^{k_{1}}\right\|_{L^{2}}$, and $\left\|f_{k_{2}}\right\|_{Z_{k_{2}}} \approx 2^{j_{2} / 2} \beta_{k_{2}, j_{2}}\left\|f_{k_{2}, j_{2}}\right\|_{L^{2}}$. The bound (7.1) which we have to prove becomes

$2^{k}\left\|\eta_{k}(\xi) \cdot(\tau-\omega(\xi)+i)^{-1} f_{k_{2}, j_{2}} * f_{0, j_{1}}^{k_{1}}\right\|_{Z_{k}} \leq C 2^{j_{1}-k_{1}}\left\|f_{0, j_{1}}^{k_{1}}\right\|_{L^{2}} \cdot 2^{j_{2} / 2} \beta_{k_{2}, j_{2}}\left\|f_{k_{2}, j_{2}}\right\|_{L^{2}}$.

Let $h_{k}(\xi, \tau)=\eta_{k}(\xi)(\tau-\omega(\xi)+i)^{-1}\left(f_{k_{2}, j_{2}} * f_{0, j_{1}}^{k_{1}}\right)(\xi, \tau)$. The first observation is that for most choices of $j_{1}$ and $j_{2}$, depending on $k$ and $k_{1}$, the function $h_{k}$ is supported in a bounded number of regions $D_{k, j}$, so (7.3) suffices to control $2^{k}\left\|h_{k}\right\|_{X_{k}}$. In view of (7.4), the function $h_{k}$ is supported in a bounded number of regions $D_{k, j}$, and (7.8) follows from (7.3), unless

$$
\left\{\begin{array}{l}
\left|j_{1}-\left(k+k_{1}\right)\right| \leq 10 \text { and } j_{2} \leq k+k_{1}+10 \text { or } \\
\left|j_{2}-\left(k+k_{1}\right)\right| \leq 10 \text { and } j_{1} \leq k+k_{1}+10 \text { or } \\
j_{1}, j_{2} \geq k+k_{1}-10 \text { and }\left|j_{1}-j_{2}\right| \leq 10 .
\end{array}\right.
$$

Assume (7.9) holds. Using (7.4), $\mathbf{1}_{D_{k, j}}(\xi, \tau) \cdot h_{k} \equiv 0$ unless $j \leq \max \left(j_{1}, j_{2}\right)+C$. We have two cases: if $j_{1} \geq k+k_{1}-20$, then, in view of (7.9), $j_{2} \leq j_{1}+C$ and the function $h_{k}$ is supported in $\bigcup_{j \leq j_{1}+C} D_{k, j}$. By (7.7),

$$
\begin{aligned}
& 2^{k}\left\|h_{k}\right\|_{X_{k}} \leq C 2^{k} \sum_{j \leq j_{1}+C} 2^{j / 2} \beta_{k, j}\left\|\eta_{j}(\tau-\omega(\xi)) h_{k}(\xi, \tau)\right\|_{L^{2}} \\
& \quad \leq C\left[\sum_{j \leq j_{1}+C} 2^{-\max \left(j, j_{2}\right) / 2}\right] 2^{-k_{1} / 2} \cdot 2^{j_{1}}\left\|f_{0, j_{1}}^{k_{1}}\right\|_{L^{2}} \cdot 2^{j_{2} / 2} \beta_{k_{2}, j_{2}}\left\|f_{k_{2}, j_{2}}\right\|_{L^{2}},
\end{aligned}
$$


which suffices for (7.8). Assume now that $j_{1} \leq k+k_{1}-20$, so, in view of (7.9), $\left|j_{2}-\left(k+k_{1}\right)\right| \leq 10$ and the function $h_{k}$ is supported in $\bigcup_{j \leq k+k_{1}+C} D_{k, j}$. Then, using Lemma 4.1(b) and (c) (in fact the proof of part (b)),

$$
\begin{aligned}
2^{k}\left\|h_{k}\right\|_{Z_{k}} & \leq C 2^{k / 2}\left\|\mathcal{F}^{-1}\left[(\tau-\omega(\xi)+i) h_{k}(\xi, \tau)\right]\right\|_{L_{x}^{1} L_{t}^{2}} \\
& \leq C 2^{k / 2}\left\|\mathcal{F}^{-1}\left(f_{0, j_{1}}^{k_{1}}\right)\right\|_{L_{x}^{2} L_{t}^{\infty}}\left\|\mathcal{F}^{-1}\left(f_{k_{2}, j_{2}}\right)\right\|_{L_{x}^{2} L_{t}^{2}} \\
& \leq C 2^{\left(j_{1}-k_{1}\right) / 2}\left\|f_{0, j_{1}}^{k_{1}}\right\|_{L^{2}} \cdot 2^{\left(k+k_{1}\right) / 2}\left\|f_{k_{2}, j_{2}}\right\|_{L^{2}},
\end{aligned}
$$

which suffices for (7.8) since $\left|j_{2}-\left(k+k_{1}\right)\right| \leq 10$. For later use we notice that we proved the slightly stronger estimate, with the factor $2^{-k_{1}}$ in the right-hand side of (7.8) replaced by $2^{-k_{1} / 2}$,

$2^{k}\left\|\eta_{k}(\xi)(\tau-\omega(\xi)+i)^{-1} f_{k_{2}, j_{2}} * f_{0, j_{1}}^{k_{1}}\right\|_{Z_{k}} \leq C 2^{j_{1}-k_{1} / 2}\left\|f_{0, j_{1}}^{k_{1}}\right\|_{L^{2}} \cdot 2^{j_{2} / 2} \beta_{k_{2}, j_{2}}|| f_{k_{2}, j_{2}} \|_{L^{2}}$.

Case 2: $f_{0}=f_{0, j_{1}}^{k_{1}}$ is supported in $D_{k_{1}, j_{1}}, j_{1} \geq 0, k_{1} \leq 1, f_{k_{2}}=g_{k_{2}}$ is supported in $\bigcup_{j_{2} \leq k_{2}-1} D_{k_{2}, j_{2}},\left\|f_{0}\right\|_{Z_{0}} \approx 2^{j_{1}-k_{1}}\left\|f_{0, j_{1}}^{k_{1}}\right\|_{L^{2}}$, and $\left\|f_{k_{2}}\right\|_{Z_{k_{2}}} \approx\left\|g_{k_{2}}\right\|_{Y_{k_{2}}}$. The bound (7.1) which we have to prove becomes

$$
2^{k}|| \eta_{k}(\xi) \cdot(\tau-\omega(\xi)+i)^{-1} g_{k_{2}} * f_{0, j_{1}}^{k_{1}}\left\|_{Z_{k}} \leq C 2^{j_{1}-k_{1}}\right\| f_{0, j_{1}}^{k_{1}}\left\|_{L^{2}} \cdot\right\| g_{k_{2}} \|_{Y_{k_{2}}} \cdot
$$

As before, let $h_{k}(\xi, \tau)=\eta_{k}(\xi)(\tau-\omega(\xi)+i)^{-1}\left(g_{k_{2}} * f_{0, j_{1}}^{k_{1}}\right)(\xi, \tau)$. In view of Lemma 4.1(b), (c), and the bound (7.10), we may assume that $g_{k_{2}}$ is supported in the set $\left\{\left(\xi_{2}, \tau_{2}\right): \xi_{2} \in I_{k_{2}},\left|\tau_{2}-\omega\left(\xi_{2}\right)\right| \leq 2^{k+k_{1}-20}\right\}$. We have two cases: if $j_{1} \geq k+k_{1}-20$, then let $g_{k_{2}, j_{2}}\left(\xi_{2}, \tau_{2}\right)=g_{k_{2}}\left(\xi_{2}, \tau_{2}\right) \eta_{j_{2}}\left(\tau_{2}-\omega\left(\xi_{2}\right)\right)$. Using $X_{k}$ norms, Lemma 4.1(b), and (7.7), the left-hand side of (7.11) is dominated by

$$
\begin{aligned}
& C \sum_{j, j_{2} \leq j_{1}+C} 2^{k} 2^{j / 2} \beta_{k, j}\left\|\eta_{k}(\xi) \eta_{j}(\tau-\omega(\xi))(\tau-\omega(\xi)+i)^{-1}\left(f_{0, j_{1}}^{k_{1}} * g_{k_{2}, j_{2}}\right)\right\|_{L^{2}} \\
& \leq C \gamma_{k, k_{1}} \cdot 2^{j_{1}}\left\|f_{0, j_{1}}^{k_{1}}\right\|_{L^{2}} \sum_{j, j_{2} \leq j_{1}+C} 2^{-\max \left(j, j_{2}\right) / 2} \cdot 2^{j_{2} / 2} \beta_{k_{2}, j_{2}}\left\|g_{k_{2}, j_{2}}\right\|_{L^{2}} \\
& \leq C \gamma_{k, k_{1}} \cdot 2^{j_{1}}\left\|f_{0, j_{1}}^{k_{1}}\right\|_{L^{2}} \cdot\left\|g_{k_{2}}\right\|_{Y_{k_{2}}},
\end{aligned}
$$

which suffices to prove (7.11) in this case. Assume now that $j_{1} \leq k+k_{1}-20$. In view of (17.4), the function in the left-hand side of (7.11) is supported in the union of a bounded number of dyadic regions $D_{k, j},\left|j-\left(k+k_{1}\right)\right| \leq C$. Then, using $X_{k}$ norms in the left-hand side of (77.11) and Lemma4.2(c), the left-hand side of (7.11) is dominated by

$$
\begin{aligned}
C 2^{k} 2^{-\left(k+k_{1}\right) / 2}\left\|f_{0, j_{1}}^{k_{1}} * g_{k_{2}}\right\|_{L^{2}} & \leq C 2^{\left(k-k_{1}\right) / 2}\left\|\mathcal{F}^{-1}\left(f_{0, j_{1}}^{k_{1}}\right)\right\|_{L_{x}^{2} L_{t}^{\infty}}\left\|\mathcal{F}^{-1}\left(g_{k_{2}}\right)\right\|_{L_{x}^{\infty} L_{t}^{2}} \\
& \leq C 2^{\left(k-k_{1}\right) / 2} \cdot 2^{j_{1} / 2}\left\|f_{0, j_{1}}^{k_{1}}\right\|_{L^{2}} \cdot 2^{-k / 2}\left\|g_{k_{2}}\right\|_{Y_{k_{2}}} \\
& \leq C 2^{\left(j_{1}-k_{1}\right) / 2}\left\|f_{0, j_{1}}^{k_{1}}\right\|_{L^{2}} \cdot\left\|g_{k_{2}}\right\|_{Y_{k_{2}}},
\end{aligned}
$$

which completes the proof of (7.11).

Case 3: $f_{0}=g_{0, j}$ is supported in $\widetilde{I}_{0} \times \widetilde{I}_{j_{1}}, j_{1} \geq 0,\left\|f_{0}\right\|_{Z_{0}} \approx 2^{j_{1}}\left\|\mathcal{F}^{-1}\left(g_{0, j_{1}}\right)\right\|_{L_{x}^{1} L_{t}^{2}}$. The bound (7.1) which we have to prove becomes

$$
2^{k}\left\|\eta_{k}(\xi) \cdot(\tau-\omega(\xi)+i)^{-1} f_{k_{2}} * g_{0, j_{1}}\right\|_{Z_{k}} \leq C 2^{j_{1}}\left\|\mathcal{F}^{-1}\left(g_{0, j_{1}}\right)\right\|_{L_{x}^{1} L_{t}^{2}} \cdot\left\|f_{k_{2}}\right\|_{Z_{k_{2}}} .
$$

Using the representation (5.12), we see easily that

$$
\left\|\mathcal{F}^{-1}\left(g_{0, j_{1}}\right)\right\|_{L_{x}^{1} L_{t}^{\infty}}+\left\|\mathcal{F}^{-1}\left(g_{0, j_{1}}\right)\right\|_{L_{x}^{2} L_{t}^{\infty}} \leq C 2^{j_{1} / 2}\left\|\mathcal{F}^{-1}\left(g_{0, j_{1}}\right)\right\|_{L_{x}^{1} L_{t}^{2}} .
$$


Thus, using the definitions, Lemma 4.1(b), (c), and Lemma 4.2(c),

$$
\begin{aligned}
2^{k} \| \eta_{k}(\xi) & \eta_{\leq k+C}(\tau-\omega(\xi))(\tau-\omega(\xi)+i)^{-1} f_{k_{2}} * g_{0, j_{1}} \|_{Z_{k}} \\
& \leq C 2^{k / 2}\left\|\mathcal{F}^{-1}\left(f_{k_{2}} * g_{0, j_{1}}\right)\right\|_{L_{x}^{1} L_{t}^{2}} \\
& \leq C 2^{k / 2}\left\|\mathcal{F}^{-1}\left(f_{k_{2}}\right)\right\|_{L_{x}^{\infty} L_{t}^{2}}\left\|\mathcal{F}^{-1}\left(g_{0, j_{1}}\right)\right\|_{L_{x}^{1} L_{t}^{\infty}} \\
& \leq C 2^{j_{1} / 2}\left\|\mathcal{F}^{-1}\left(g_{0, j_{1}}\right)\right\|_{L_{x}^{1} L_{t}^{2}} \cdot\left\|f_{k_{2}}\right\|_{Z_{k_{2}}} .
\end{aligned}
$$

Thus, for (7.12), it suffices to prove that

$$
\begin{aligned}
& 2^{k} \sum_{j \geq k+C} 2^{-j / 2} \beta_{k, j}\left\|\eta_{k}(\xi) \eta_{j}(\tau-\omega(\xi)) f_{k_{2}} * g_{0, j_{1}}\right\|_{L^{2}} \\
& \quad \leq C 2^{j_{1}}\left\|\mathcal{F}^{-1}\left(g_{0, j_{1}}\right)\right\|_{L_{x}^{1} L_{t}^{2}} \cdot\left\|f_{k_{2}}\right\|_{Z_{k_{2}}} .
\end{aligned}
$$

Using Lemma 4.2(c) and (7.13) again,

$$
\begin{aligned}
\left\|\eta_{k}(\xi) \eta_{j}(\tau-\omega(\xi)) f_{k_{2}} * g_{0, j_{1}}\right\|_{L^{2}} & \leq C\left\|\mathcal{F}^{-1}\left(f_{k_{2}}\right)\right\|_{L_{x}^{\infty} L_{t}^{2}}\left\|\mathcal{F}^{-1}\left(g_{0, j_{1}}\right)\right\|_{L_{x}^{2} L_{t}^{\infty}} \\
& \leq C 2^{j_{1} / 2}\left\|\mathcal{F}^{-1}\left(g_{0, j_{1}}\right)\right\|_{L_{x}^{1} L_{t}^{2}} \cdot 2^{-k / 2}\left\|f_{k_{2}}\right\|_{Z_{k_{2}}} .
\end{aligned}
$$

We use this bound to control the sum over $j \leq 2 k+j_{1}+C$ in (7.14). For $j \geq$ $2 k+j_{1}+C, 2^{-j / 2} \beta_{k, j} \approx 2^{-k}$, and for (7.12), it suffices to prove that

$$
\sum_{j \geq 2 k+j_{1}+C}\left\|\eta_{k}(\xi) \eta_{j}(\tau-\omega(\xi)) f_{k_{2}} * g_{0, j_{1}}\right\|_{L^{2}} \leq C 2^{j_{1}}\left\|\mathcal{F}^{-1}\left(g_{0, j_{1}}\right)\right\|_{L_{x}^{1} L_{t}^{2}} \cdot\left\|f_{k_{2}}\right\|_{Z_{k_{2}}} .
$$

By examining the supports of the functions, $\eta_{k}(\xi) \eta_{j}(\tau-\omega(\xi)) f_{k_{2}} * g_{0, j_{1}} \equiv 0$ if $f_{k_{2}} \in Y_{k_{2}}$ and $j \geq 2 k+j_{1}+C$. So, in (7.15), we may assume $f_{k_{2}}=f_{k_{2}, j_{2}}$ is supported in $D_{k_{2}, j_{2}}, j_{2} \geq 2 k+j_{1}+C$. The sum in $j$ in (7.15) is taken over $\left|j-j_{2}\right| \leq C$. Using Lemma $4.2(\mathrm{c})$ and (7.13), the left-hand side of (7.15) is dominated by

$$
C\left\|\mathcal{F}^{-1}\left(f_{k_{2}, j_{2}}\right)\right\|_{L_{x}^{\infty} L_{t}^{2}}\left\|\mathcal{F}^{-1}\left(g_{0, j_{1}}\right)\right\|_{L_{x}^{2} L_{t}^{\infty}} \leq C 2^{j_{1} / 2}\left\|\mathcal{F}^{-1}\left(g_{0, j_{1}}\right)\right\|_{L_{x}^{1} L_{t}^{2}} \cdot\left\|f_{k_{2}, j_{2}}\right\|_{Z_{k_{2}}} .
$$

This completes the proof of (7.15) and (7.12).

For later use, we notice that a simplified version of our argument can be used to prove the following: if $k \geq 20, k_{2} \in[k-2, k+2], f_{k_{2}} \in Z_{k_{2}}$, and $f_{0} \in \bar{Z}_{0}$, then

$$
\left\|\eta_{k}(\xi) \cdot(\tau-\omega(\xi)+i)^{-1} f_{k_{2}} * f_{0}\right\|_{Z_{k}} \leq C\left\|f_{k_{2}}\right\|_{Z_{k_{2}}}\left\|f_{0}\right\|_{\bar{Z}_{0}} \text {. }
$$

To prove (7.16), we use Lemma 4.1(b) to bound $\left\|f_{k_{2}}\left|Z_{z_{2}} \geq C^{-1} k^{-1}\right| \mid f_{k_{2}}\right\|_{X_{k_{2}}}$. Then, we write $f_{0}=\sum_{j_{1} \geq 0} \sum_{k_{1} \leq 1} f_{k_{1}, j_{1}}, f_{k_{1}, j_{1}}$ supported in $D_{k_{1}, j_{1}}$ and $\left\|f_{0}\right\|_{\bar{Z}_{0}} \geq$ $\sum_{j_{1} \geq 0} \sum_{k_{1} \leq 1} 2^{j_{1}} 2^{k_{1} / 4}\left\|f_{k_{1}, j_{1}}\right\|_{L^{2}}$. In view of the definitions, for (7.16) it suffices to prove that if $f_{k_{2}, j_{2}}$ is supported in $D_{k_{2}, j_{2}}$, then

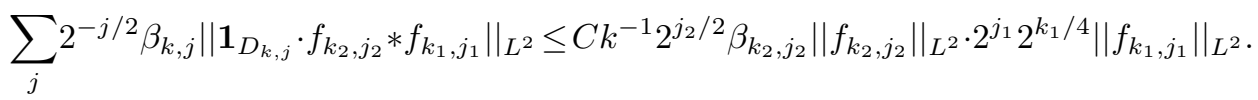

Using (6.19), we bound $\left\|\mathbf{1}_{D_{k, j}} \cdot f_{k_{2}, j_{2}} * f_{k_{1}, j_{1}}\right\|_{L^{2}} \leq C 2^{k_{1} / 2} 2^{j_{1} / 2}\left\|f_{k_{2}, j_{2}}\right\|_{L^{2}} \cdot \mid f_{k_{1}, j_{1}} \|_{L^{2}}$. So, it suffices to prove that

$$
2^{k_{1} / 4} k \sum_{j} 2^{-j / 2} \beta_{k, j} \leq C 2^{\left(j_{1}+j_{2}\right) / 2},
$$

where the sum is taken over $j$ satisfying (7.4). This follows easily by examining the cases $\max \left(j_{1}, j_{2}\right) \leq k+k_{1}-20$ and $\max \left(j_{1}, j_{2}\right) \geq k+k_{1}-20$ (in the second case we estimate $\left.2^{-j / 2} \beta_{k, j} \leq C\right)$. 
Proof of Proposition 7.2. The proof is similar to the proof of Proposition 7.1, with an additional technical difficulty related to the sum in $k_{1}$ in the left-hand side of (7.2). Our main tools are the bounds (7.3) (with $\gamma_{k, k_{1}} \approx 2^{-k_{1} / 2}$ if $k_{1} \geq 1$ ) and (7.6). For any $k_{1} \in[1, k-10]$ we decompose

$$
f_{k_{1}}=f_{k_{1}}^{h}+f_{k_{1}}^{l}=f_{k_{1}} \cdot\left[1-\eta_{\leq k+k_{1}-20}(\tau-\omega(\xi))\right]+f_{k_{1}} \cdot \eta_{\leq k+k_{1}-20}(\tau-\omega(\xi)) .
$$

We show first that

$$
2^{k}\left\|\eta_{k}(\xi)(\tau-\omega(\xi)+i)^{-1} f_{k_{2}} * f_{k_{1}}^{h}\right\|_{X_{k}} \leq C 2^{-k_{1} / 4}\left\|f_{k_{2}}\right\|_{Z_{k_{2}}}\left\|f_{k_{1}}^{h}\right\|_{Z_{k_{1}}} .
$$

Assuming (7.18), we can use the factor $2^{-k_{1} / 4}$ to sum in $k_{1}$ and obtain

$$
2^{k}\left\|\eta_{k}(\xi)(\tau-\omega(\xi)+i)^{-1} f_{k_{2}} * \sum_{k_{1}=1}^{k-10} f_{k_{1}}^{h}\right\|_{X_{k}} \leq C\left\|f_{k_{2}}\right\|_{Z_{k_{2}}} \sup _{k_{1} \in[1, k-10]}\left\|f_{k_{1}}\right\|_{Z_{k_{1}}} .
$$

To prove (7.18), we use the representation (4.1) and (7.6). We may assume $f_{k_{1}}^{h}=$ $f_{k_{1}, j_{1}}$ is supported in $D_{k_{1}, j_{1}}, j_{1} \geq k+k_{1}-20,\left\|f_{k_{1}}^{h}\right\|_{Z_{k_{1}}} \approx 2^{j_{1} / 2} \beta_{k_{1}, j_{1}}|| f_{k_{1}, j_{1}} \|_{L^{2}}$. We have two cases: if $f_{k_{2}}=f_{k_{2}, j_{2}}$ is supported in $D_{k_{2}, j_{2}}, j_{2} \geq 0,\left\|f_{k_{2}}\right\|_{Z_{k_{2}}} \approx$ $2^{j_{2} / 2} \beta_{k_{2}, j_{2}}\left\|f_{k_{2}, j_{2}}\right\|_{L^{2}}$, then, using (7.6) and the definitions, the left-hand side of (17.18) is dominated by

$$
\begin{aligned}
C\left[\sum_{j}\left(2^{k_{1} / 2}+2^{j / 4}\right)^{-1}\right] & \cdot 2^{j_{1} / 2} \beta_{k_{1}, j_{1}}\left\|f_{k_{1}, j_{1}}\right\|_{L^{2}} \cdot 2^{j_{2} / 2} \beta_{k_{2}, j_{2}}|| f_{k_{2}, j_{2}} \|_{L^{2}} \\
& \leq C 2^{-k_{1} / 4} \cdot 2^{j_{1} / 2} \beta_{k_{1}, j_{1}}\left\|f_{k_{1}, j_{1}}\right\|_{L^{2}} \cdot 2^{j_{2} / 2} \beta_{k_{2}, j_{2}}|| f_{k_{2}, j_{2}} \|_{L^{2}},
\end{aligned}
$$

which gives (7.18) in this case. If $f_{k_{2}}=g_{k_{2}}$ is supported in $\bigcup_{j_{2} \leq k_{2}-1} D_{k_{2}, j_{2}}$, $\left\|f_{k_{2}}\right\|_{Z_{k_{2}}} \approx\left\|g_{k_{2}}\right\|_{Y_{k_{2}}}$, then let $g_{k_{2}, j_{2}}\left(\xi_{2}, \tau_{2}\right)=g_{k_{2}}\left(\xi_{2}, \tau_{2}\right) \eta_{j_{2}}\left(\tau_{2}-\omega\left(\xi_{2}\right)\right)$. In view of Lemma 4.1(b), (7.4), and (7.6), the left-hand side of (7.18) is dominated by

$$
\begin{aligned}
& C \sum_{j, j_{2} \leq j_{1}+C} 2^{k} 2^{j / 2} \beta_{k, j}\left\|\eta_{k}(\xi) \eta_{j}(\tau-\omega(\xi))(\tau-\omega(\xi)+i)^{-1}\left(g_{k_{2}, j_{2}} * f_{k_{1}, j_{1}}\right)\right\|_{L^{2}} \\
& \leq C 2^{j_{1} / 2} \beta_{k_{1}, j_{1}}\left\|f_{k_{1}, j_{1}}\right\|_{L^{2}} \sum_{j, j_{2} \leq j_{1}+C}\left(2^{k_{1} / 2}+2^{\max \left(j, j_{2}\right) / 4}\right)^{-1} 2^{j_{2} / 2}\left\|g_{k_{2}, j_{2}}\right\|_{L^{2}} \\
& \leq C 2^{-k_{1} / 4} \cdot 2^{j_{1} / 2} \beta_{k_{1}, j_{1}}\left\|f_{k_{1}, j_{1}}\right\|_{L^{2}} \cdot\left\|g_{k_{2}}\right\|_{Y_{k_{2}}},
\end{aligned}
$$

which completes the proof of (7.18).

In view of (7.19), for (7.2) it suffices to prove that

$$
2^{k}\left\|\eta_{k}(\xi)(\tau-\omega(\xi)+i)^{-1} f_{k_{2}} * \sum_{k_{1}=1}^{k-10} f_{k_{1}}^{l}\right\|_{Z_{k}} \leq C\left\|f_{k_{2}}\right\|_{Z_{k_{2}}} \sup _{k_{1} \in[1, k-10]}\left\|\left(I-\partial_{\tau}^{2}\right) f_{k_{1}}^{l}\right\|_{Z_{k_{1}}}
$$

for any functions $f_{k_{1}}^{l}$ supported in $\bigcup_{j_{1} \leq k+k_{1}-19} D_{k_{1}, j_{1}}$. Using the representation (4.1), we analyze two cases. 
Case 1: $f_{k_{2}}=f_{k_{2}, j_{2}}$ is supported in $D_{k_{2}, j_{2}},\left\|f_{k_{2}}\right\|_{Z_{k_{2}}} \approx 2^{j_{2} / 2} \beta_{k_{2}, j_{2}}|| f_{k_{2}, j_{2}} \|_{L^{2}}$, $j_{2} \geq 0$. The bound (7.20) which we have to prove becomes

$$
\begin{aligned}
& 2^{k}\left\|\eta_{k}(\xi)(\tau-\omega(\xi)+i)^{-1} f_{k_{2}, j_{2}} * \sum_{k_{1}=1}^{k-10} f_{k_{1}}^{l}\right\|_{Z_{k}} \\
& \quad \leq C 2^{j_{2} / 2} \beta_{k_{2}, j_{2}}\left\|f_{k_{2}, j_{2}}\right\|_{L^{2}} \sup _{k_{1} \in[1, k-10]}\left\|\left(I-\partial_{\tau}^{2}\right) f_{k_{1}}^{l}\right\|_{Z_{k_{1}}},
\end{aligned}
$$

for any functions $f_{k_{1}}^{l}$ supported in $\bigcup_{j_{1} \leq k+k_{1}-19} D_{k_{1}, j_{1}}$. Notice that $j_{2}$ is fixed in (7.21). We divide the set of indices $k_{1}$ into two sets:

$$
\left\{\begin{array}{l}
A_{k, j_{2}}=\left\{k_{1} \in[1, k-10]:\left|k+k_{1}-j_{2}\right| \leq 15\right\} ; \\
B_{k, j_{2}}=\left\{k_{1} \in[1, k-10]:\left|k+k_{1}-j_{2}\right| \geq 16\right\} .
\end{array}\right.
$$

The set $A_{k, j_{2}}$ has at most 31 elements, so, for (7.21) it suffices to prove that

$2^{k}\left\|\eta_{k}(\xi)(\tau-\omega(\xi)+i)^{-1} f_{k_{2}, j_{2}} * f_{k_{1}}^{l}\right\|_{Z_{k}} \leq C 2^{j_{2} / 2} \beta_{k_{2}, j_{2}}\left\|f_{k_{2}, j_{2}}\right\|\left\|_{L^{2}}\right\|\left(I-\partial_{\tau}^{2}\right) f_{k_{1}}^{l} \|_{Z_{k_{1}}}$ for $k_{1} \in A_{k, j_{2}}$ and

$2^{k}\left\|\eta_{k}(\xi)(\tau-\omega(\xi)+i)^{-1} f_{k_{2}, j_{2}} * f_{k_{1}}^{l}\right\|_{X_{k}} \leq C 2^{-k_{1} / 4} \cdot 2^{j_{2} / 2} \beta_{k_{2}, j_{2}}\left\|f_{k_{2}, j_{2}}\right\|\left\|_{L^{2}}\right\| f_{k_{1}}^{l} \|_{Z_{k_{1}}}$ for $k_{1} \in B_{k, j_{2}}$.

We prove first (7.22). In view of the restriction on the support of $f_{k_{1}}^{l}$, the condition $k_{1} \in A_{k, j_{2}}$, and (7.4), the function $\eta_{k}(\xi)(\tau-\omega(\xi)+i)^{-1} f_{k_{2}, j_{2}} * f_{k_{1}}^{l}$ is supported in $\bigcup_{j \leq k+k_{1}+C} D_{k, j}$. In view of the definition of the space $Z_{k}$, for (7.22) it suffices to prove that

$$
\begin{aligned}
& 2^{k}\left\|\eta_{\leq k-1}(\tau-\omega(\xi)) \eta_{k}(\xi)(\tau-\omega(\xi)+i)^{-1} f_{k_{2}, j_{2}} * f_{k_{1}}^{l}\right\|_{Y_{k}} \\
& \quad \leq C 2^{j_{2} / 2} \beta_{k_{2}, j_{2}}|| f_{k_{2}, j_{2}}\left\|_{L^{2}}\right\|\left(I-\partial_{\tau}^{2}\right) f_{k_{1}}^{l} \|_{Z_{k_{1}}}
\end{aligned}
$$

and

$$
\begin{gathered}
2^{k} \sum_{j=k}^{k+k_{1}+C} 2^{j / 2} \beta_{k, j}\left\|\eta_{j}(\tau-\omega(\xi)) \eta_{k}(\xi)(\tau-\omega(\xi)+i)^{-1} f_{k_{2}, j_{2}} * f_{k_{1}}^{l}\right\|_{L^{2}} \\
\leq C 2^{j_{2} / 2} \beta_{k_{2}, j_{2}}\left\|f_{k_{2}, j_{2}}\right\|_{L^{2}}\left\|f_{k_{1}}^{l}\right\|_{Z_{k_{1}}} .
\end{gathered}
$$

For (7.24) we use Lemma 4.1(a), (c), and Lemma 4.2(b). Since $\left|k+k_{1}-j_{2}\right| \leq 10$, the left-hand side of (7.24) is dominated by

$$
\begin{aligned}
C 2^{k / 2}\left\|\mathcal{F}^{-1}\left(f_{k_{2}, j_{2}} * f_{k_{1}}^{l}\right)\right\|_{L_{x}^{1} L_{t}^{2}} & \leq C 2^{k / 2}\left\|\mathcal{F}^{-1}\left(f_{k_{2}, j_{2}}\right)\right\|_{L^{2}}\left\|\mathcal{F}^{-1}\left(f_{k_{1}}^{l}\right)\right\|_{L_{x}^{2} L_{t}^{\infty}} \\
& \leq C 2^{k / 2}\left\|f_{k_{2}, j_{2}}\right\|_{L^{2}} \cdot 2^{k_{1} / 2}\left\|\left(I-\partial_{\tau}^{2}\right) f_{k_{1}}^{l}\right\|_{Z_{k_{1}}},
\end{aligned}
$$

which completes the proof of (7.24). For (7.25), we notice that the sum in the left-hand side contains at most $k_{1}+C$ terms. In addition, using Lemma 4.1(b), $\left\|f_{k_{1}}^{l}\right\|_{Z_{k_{1}}} \geq C k_{1}^{-1}\left\|f_{k_{1}}^{l}\right\|_{X_{k_{1}}}$, and, using (7.3), for any $j \in\left[k, k+k_{1}+C\right]$

$$
\begin{gathered}
2^{k} 2^{j / 2} \beta_{k, j}\left\|\eta_{j}(\tau-\omega(\xi)) \eta_{k}(\xi)(\tau-\omega(\xi)+i)^{-1} f_{k_{2}, j_{2}} * f_{k_{1}}^{l}\right\|_{L^{2}} \\
\leq C 2^{-k_{1} / 2} 2^{j_{2} / 2} \beta_{k_{2}, j_{2}}\left\|f_{k_{2}, j_{2}}\right\|_{L^{2}} \cdot\left\|f_{k_{1}}^{l}\right\|_{X_{k_{1}}} .
\end{gathered}
$$

This completes the proof of (7.25) and (7.22). 
We prove now the bound (7.23). The main observation is that the function $\eta_{k}(\xi)(\tau-\omega(\xi)+i)^{-1} f_{k_{2}, j_{2}} * f_{k_{1}}^{l}$ is supported in a bounded number of regions $D_{k, j}$ (assuming $j_{2}$ and $k_{1}$ fixed). This is due to the support property of the function $f_{k_{1}}^{l}$, the assumption $\left|k+k_{1}-j_{2}\right| \geq 16$, and (7.4). Thus, using (7.3), the left-hand side of (7.23) is dominated by

$$
\begin{gathered}
C \sup _{j} 2^{k} 2^{j / 2} \beta_{k, j}\left\|\eta_{j}(\tau-\omega(\xi)) \eta_{k}(\xi)(\tau-\omega(\xi)+i)^{-1} f_{k_{2}, j_{2}} * f_{k_{1}}^{l}\right\|_{L^{2}} \\
\leq C 2^{-k_{1} / 2} 2^{j_{2} / 2} \beta_{k_{2}, j_{2}}\left\|f_{k_{2}, j_{2}}\right\|_{L^{2}} \cdot\left\|f_{k_{1}}^{l}\right\|_{X_{k_{1}}},
\end{gathered}
$$

which suffices for (7.23) since $\left\|f_{k_{1}}^{l}\right\|_{Z_{k_{1}}} \geq C k_{1}^{-1}|| f_{k_{1}}^{l} \|_{X_{k_{1}}}$ (see Lemma 4.1(b)).

Case 2: $f_{k_{2}}=g_{k_{2}}$ is supported in $\bigcup_{j_{2} \leq k_{2}-20} D_{k_{2}, j_{2}},\left\|f_{k_{2}}\right\|_{Z_{k_{2}}} \approx\left\|g_{k_{2}}\right\|_{Y_{k_{2}}}$. The bound (7.20) which we have to prove becomes

$$
2^{k}\left\|\eta_{k}(\xi)(\tau-\omega(\xi)+i)^{-1} g_{k_{2}} * \sum_{k_{1}=1}^{k-10} f_{k_{1}}^{l}\right\|_{Z_{k}} \leq C\left\|g_{k_{2}}\right\|_{Y_{k_{2}}} \sup _{k_{1} \in[1, k-10]}\left\|\left(I-\partial_{\tau}^{2}\right) f_{k_{1}}^{l}\right\|_{Z_{k_{1}}}
$$

for any functions $f_{k_{1}}^{l}$ supported in $\bigcup_{j_{1} \leq k+k_{1}-19} D_{k_{1}, j_{1}}$. Using Lemma 4.1(b) again, it suffices to prove that

$$
2^{k}\left\|\eta_{k}(\xi)(\tau-\omega(\xi)+i)^{-1} g_{k_{2}} * f_{k_{1}}^{l}\right\|_{X_{k}} \leq C 2^{-k_{1} / 2}\left\|g_{k_{2}}\right\|_{Y_{k_{2}}}\left\|f_{k_{1}}^{l}\right\|_{X_{k_{1}}} .
$$

Using (7.4) and the support properties of $g_{k_{2}}$ and $f_{k_{1}}^{l}, \eta_{k}(\xi)(\tau-\omega(\xi)+i)^{-1} g_{k_{2}} * f_{k_{1}}^{l}$ is supported in a bounded number of regions $D_{k, j},\left|k+k_{1}-j\right| \leq C$. Thus, for (7.26) it suffices to prove that if $f_{k_{1}, j_{1}}$ is supported in $D_{k_{1}, j_{1}}, j_{1} \leq k+k_{1}-19$ and $\left|j-k-k_{1}\right| \leq C$, then

$$
2^{k / 2}\left\|\left.\mathbf{1}_{D_{k, j}} \cdot\left(g_{k_{2}} * f_{k_{1}, j_{1}}\right)\right|_{L^{2}} \leq C\right\| g_{k_{2}}\left\|_{Y_{k_{2}}} \cdot 2^{j_{1} / 2}\right\| f_{k_{1}, j_{1}} \|_{L^{2}}
$$

To prove (7.27), we may assume $k_{2} \geq 100$. For $j_{2} \leq k_{2}$ let $g_{k_{2}, j_{2}}(\xi, \tau)=$ $\eta_{j_{2}}(\tau-\omega(\xi)) g_{k_{2}}(\xi, \tau)$. Notice that in view of (6.20) and Lemma 4.1(b)

$$
\begin{aligned}
2^{k / 2}\left\|\mathbf{1}_{D_{k, j}} \cdot\left(g_{k_{2}, j_{2}} * f_{k_{1}, j_{1}}\right)\right\|_{L^{2}} & \leq C 2^{j_{2} / 2}\left\|g_{k_{2}, j_{2}}\right\|_{L^{2}} \cdot 2^{j_{1} / 2}\left\|f_{k_{1}, j_{1}}\right\|_{L^{2}} \\
& \leq C\left\|g_{k_{2}}\right\|_{Y_{k_{2}}} \cdot 2^{j_{1} / 2}\left\|f_{k_{1}, j_{1}}\right\|_{L^{2}},
\end{aligned}
$$

for any $j_{2} \leq k_{2}$. To prove (7.27), we have to avoid the logarithmic divergence that appears when summing the bound (7.28) over $j_{2} \leq k_{2}$. In view of (4.6) and the Minkowski inequality (see the explanation preceding (4.17)), we may assume

$$
\left\{\begin{array}{l}
g_{k_{2}}(\xi, \tau)=2^{k_{2} / 2} \chi_{\left[k_{2}-1, k_{2}+1\right]}(\xi)(\tau-\omega(\xi)+i)^{-1} \eta_{\leq k_{2}}(\tau-\omega(\xi)) h(\tau) ; \\
\left\|g_{k_{2}}\right\|_{Y_{k_{2}}}=C\|h\|_{L_{\tau}^{2}} .
\end{array}\right.
$$

We argue as in the proof of Lemma 4.2(b). Let $h_{+}=h \cdot \mathbf{1}_{[0, \infty)}, h_{-}=h \cdot \mathbf{1}_{(-\infty, 0]}$, and define the corresponding functions $g_{k_{2},+}$ and $g_{k_{2},-}$ as in (7.29). By symmetry, it suffices to prove the bound (7.27) for the function $g_{k_{2},+}$, which is supported in the set $\left\{(\xi, \tau): \xi \in\left[-2^{k_{2}+2},-2^{k_{2}-2}\right], \tau \in\left[2^{2 k_{2}-10}, 2^{2 k_{2}+10}\right]\right\}$. In view of (3.1), $\tau-\omega(\xi)=\tau-\xi^{2}$ on the support of $g_{k_{2},+}$, and $g_{k_{2},+}(\xi, \tau)=0$ unless $|\sqrt{\tau}+\xi| \leq C$. Let

$$
\begin{gathered}
g_{k_{2},+}^{\prime}(\xi, \tau)=2^{k_{2} / 2} \chi_{\left[k_{2}-1, k_{2}+1\right]}(-\sqrt{\tau})\left(\tau-\xi^{2}+(\sqrt{\tau}+\xi)^{2}+i \sqrt{\tau} 2^{-k_{2}}\right)^{-1} \\
\times \eta_{0}(\sqrt{\tau}+\xi) \cdot h_{+}(\tau) .
\end{gathered}
$$


Using Lemma 4.1(b), it is easy to see that $\left\|g_{k_{2},+}-g_{k_{2},+}^{\prime}\right\|\left\|_{X_{k_{2}}} \leq C\right\| h_{+} \|_{L^{2}}$. In view of (7.28), for (7.27) it suffices to prove that

$$
2^{k / 2}\left\|g_{k_{2},+}^{\prime} * f_{k_{1}, j_{1}}\right\|_{L^{2}} \leq C|| h_{+}\left\|_{L^{2}} \cdot 2^{j_{1} / 2}|| f_{k_{1}, j_{1}}\right\|_{L^{2}} .
$$

We substitute the formula (7.30) and make the change of variables $\xi_{2}=-\sqrt{\tau_{2}}+\mu_{2}$. The left-hand side of (7.31) is dominated by

$$
\left\|\int_{\mathbb{R}^{2}} f_{k_{1}, j_{1}}\left(\xi+\sqrt{\tau_{2}}-\mu_{2}, \tau-\tau_{2}\right) \cdot \eta_{0}\left(\mu_{2}\right) \frac{1}{\mu_{2}+i / 2^{k_{2}+1}} \cdot h_{+}^{\prime}\left(\tau_{2}\right) d \mu_{2} d \tau_{2}\right\|_{L_{\xi, \tau}^{2}},
$$

where $h_{+}^{\prime}\left(\tau_{2}\right)=h_{+}\left(\tau_{2}\right) \chi_{\left[k_{2}-1, k_{2}+1\right]}\left(-\sqrt{\tau_{2}}\right)\left(2^{k} / \sqrt{\tau_{2}}\right)$ is supported in $\left[2^{2 k_{2}-4}, 2^{2 k_{2}+4}\right]$, $\left\|h_{+}^{\prime}\right\|_{L^{2}} \approx\left\|h_{+}\right\|_{L^{2}}$. By duality, for (7.31) it suffices to prove that for any $m \in L^{2}$

$$
\begin{aligned}
& \mid \int_{\mathbb{R}^{4}} f_{k_{1}, j_{1}}\left(\xi_{1}, \tau_{1}\right) h_{+}^{\prime}\left(\tau_{2}\right) \cdot \eta_{0}\left(\mu_{2}\right) \frac{1}{\mu_{2}+i / 2^{k_{2}+1}} \\
& \quad \times m\left(\xi_{1}-\sqrt{\tau_{2}}+\mu_{2}, \tau_{1}+\tau_{2}\right) d \mu_{2} d \tau_{2} d \xi_{1} d \tau_{1} \mid \leq C\|m\|\left\|_{L^{2}}\right\| h_{+}^{\prime}\left\|_{L^{2}} 2^{j_{1} / 2}\right\| f_{k_{1}, j_{1}} \|_{L^{2}} .
\end{aligned}
$$

Let $\widetilde{m}(\xi, \tau)=\int_{\mathbb{R}} m\left(\xi+\mu_{2}, \tau\right) \eta_{0}\left(\mu_{2}\right)\left(\mu_{2}+i / 2^{k_{2}+1}\right)^{-1} d \mu_{2},\|\widetilde{m}\|_{L^{2}} \leq C\|m\|_{L^{2}}$. In the left-hand side of the expression above we make the change of variable $\tau_{1}=$ $\mu_{1}+\omega\left(\xi_{1}\right), f_{k_{1}, j_{1}}^{\#}\left(\xi_{1}, \mu_{1}\right)=f_{k_{1}, j_{1}}\left(\xi_{1}, \mu_{1}+\omega\left(\xi_{1}\right)\right)$. It suffices to prove that

$$
\begin{gathered}
\left|\int_{\mathbb{R}^{3}} f_{k_{1}, j_{1}}^{\#}\left(\xi_{1}, \mu_{1}\right) h_{+}^{\prime}\left(\tau_{2}\right) \cdot \widetilde{m}\left(\xi_{1}-\sqrt{\tau_{2}}, \mu_{1}+\omega\left(\xi_{1}\right)+\tau_{2}\right) d \tau_{2} d \xi_{1} d \mu_{1}\right| \\
\leq C\|\widetilde{m}\|_{L^{2}}\left\|h_{+}^{\prime}\right\|_{L^{2}} \cdot 2^{j_{1} / 2}\left\|f_{k_{1}, j_{1}}^{\#}\right\|_{L^{2}} .
\end{gathered}
$$

The integral in the left-hand side of (7.32) is over the set

$$
\left(\xi_{1}, \mu_{1}, \tau_{2}\right) \in \widetilde{I}_{k_{1}} \times \widetilde{I}_{j_{1}} \times\left[2^{2 k_{2}-4}, 2^{2 k_{2}+4}\right] .
$$

Using the Hölder inequality, for (7.32) it suffices to prove that

$$
\sup _{\mu_{1} \in \mathbb{R}} \int_{\widetilde{I}_{k_{1}} \times\left[2^{2 k_{2}-4}, 2^{2 k_{2}+4}\right]}\left|\widetilde{m}\left(\xi_{1}-\sqrt{\tau_{2}}, \mu_{1}+\omega\left(\xi_{1}\right)+\tau_{2}\right)\right|^{2} d \tau_{2} d \xi_{1} \leq C|| \widetilde{m} \|_{L^{2}}^{2},
$$

which is easy to see by changing variables and recalling that $k_{1} \leq k_{2}-8$. This completes the proof of (7.27).

\section{Bilinear estimates II}

In this section we prove two bilinear estimates, which correspond to High $\times$ High $\rightarrow$ Low interactions.

Proposition 8.1. Assume $k, k_{1}, k_{2} \in \mathbb{Z}_{+}$have the property that $\max \left(k, k_{1}, k_{2}\right) \leq$ $\min \left(k, k_{1}, k_{2}\right)+30, f_{k_{1}} \in Z_{k_{1}}$, and $f_{k_{2}} \in Z_{k_{2}}$. Then

$$
\left.2^{k}\left\|\eta_{k}(\xi) \cdot A_{k}(\xi, \tau)^{-1} f_{k_{1}} * f_{k_{2}}\right\|\right|_{Z_{k}} \leq C|| f_{k_{1}}\left\|_{Z_{k_{1}}}\right\| f_{k_{2}} \|_{Z_{k_{2}}} .
$$

Moreover, any spaces $Z_{0}$ in the right-hand side of (8.1) can be replaced with $\bar{Z}_{0}$.

Proposition 8.2. Assume $k, k_{1}, k_{2} \in \mathbb{Z}_{+}, k_{1}, k_{2} \geq k+10,\left|k_{1}-k_{2}\right| \leq 2, f_{k_{1}} \in Z_{k_{1}}$, and $f_{k_{2}} \in Z_{k_{2}}$. Then

$$
\left\|\xi \cdot \eta_{k}(\xi) \cdot A_{k}(\xi, \tau)^{-1} f_{k_{1}} * f_{k_{2}}\right\|_{X_{k}} \leq C 2^{-k / 4}|| f_{k_{1}}\left\|_{Z_{k_{1}}}\right\| f_{k_{2}} \|_{Z_{k_{2}}} .
$$

The main ingredients in the proofs of Propositions 8.1 and 8.2 are the definitions, the representations (4.1) and (4.2), Lemma 4.1, and Corollary 6.2. 
Proof of Proposition 8.1. We analyze two cases.

Case 1: $\min \left(k, k_{1}, k_{2}\right) \geq 200$. In this case we prove the (stronger) bound (8.1) with the space $Z_{k}$ replaced by $X_{k}$ in the left-hand side. We show first that if $j_{1}, j_{2} \geq 0, f_{k_{1}, j_{1}}$ is an $L^{2}$ function supported in $D_{k_{1}, j_{1}}$, and $f_{k_{2}, j_{2}}$ is an $L^{2}$ function supported in $D_{k_{2}, j_{2}}$, then

$$
\begin{array}{r}
2^{k} \sum_{j} 2^{j / 2} \beta_{k, j}\left\|\eta_{k}(\xi) \eta_{j}(\tau-\omega(\xi))(\tau-\omega(\xi)+i)^{-1}\left(f_{k_{1}, j_{1}} * f_{k_{2}, j_{2}}\right)\right\|_{L^{2}} \\
\leq C \gamma\left(j_{1}, j_{2}, k\right) 2^{j_{1} / 2} \beta_{k_{1}, j_{1}}\left\|f_{k_{1}, j_{1}}\right\|_{L^{2}} \cdot 2^{j_{2} / 2} \beta_{k_{2}, j_{2}}\left\|f_{k_{2}, j_{2}}\right\|_{L^{2}}
\end{array}
$$

where

$$
\gamma\left(j_{1}, j_{2}, k\right)= \begin{cases}2^{-\max \left(j_{1}, j_{2}\right) / 4} & \text { if } \max \left(j_{1}, j_{2}\right) \leq 2 k-80 \\ 2^{-\min \left(j_{1}, j_{2}\right) / 8} & \text { if } \max \left(j_{1}, j_{2}\right) \geq 2 k-80\end{cases}
$$

To prove (8.3), we notice that, in view of (6.23),

$$
\eta_{k}(\xi) \eta_{j}(\tau-\omega(\xi))(\tau-\omega(\xi)+i)^{-1}\left(f_{k_{1}, j_{1}} * f_{k_{2}, j_{2}}\right) \equiv 0
$$

unless

$$
\left\{\begin{array}{l}
\max \left(j, j_{1}, j_{2}\right) \in[2 k-70,2 k+70] \text { or } \\
\max \left(j, j_{1}, j_{2}\right) \geq 2 k+70 \text { and } \max \left(j, j_{1}, j_{2}\right)-\operatorname{med}\left(j, j_{1}, j_{2}\right) \leq 10
\end{array}\right.
$$

We notice that for $j, j_{1}, j_{2}$ as in (8.5), $\beta_{k, j} \leq C \beta_{k_{1}, j_{1}} \beta_{k_{2}, j_{2}}$. Also, using (6.21),

$$
\begin{aligned}
& \left\|\eta_{k}(\xi) \eta_{j}(\tau-\omega(\xi))(\tau-\omega(\xi)+i)^{-1}\left(f_{k_{1}, j_{1}} * f_{k_{2}, j_{2}}\right)\right\|_{L^{2}} \\
& \quad \leq C 2^{-j} 2^{\left(j+j_{1}+j_{2}\right) / 2} 2^{-\max \left(j, j_{1}, j_{2}\right) / 2} 2^{-\operatorname{med}\left(j, j_{1}, j_{2}\right) / 4}\left\|f_{k_{1}, j_{1}}\right\|\left\|_{L^{2}}\right\| f_{k_{2}, j_{2}} \|_{L^{2}} .
\end{aligned}
$$

Thus, for (8.3), it suffices to prove that

$$
2^{k} \sum_{j} 2^{-\max \left(j, j_{1}, j_{2}\right) / 2} 2^{-\operatorname{med}\left(j, j_{1}, j_{2}\right) / 4} \leq C \gamma\left(j_{1}, j_{2}, k\right),
$$

where the sum in (8.6) is taken over $j$ satisfying (8.5). If $\max \left(j_{1}, j_{2}\right) \leq 2 k-80$, then $j \in[2 k-70,2 k+70]$ and the bound (8.6) follows easily from the definition (8.4). If $j_{1}=\max \left(j_{1}, j_{2}\right) \geq 2 k-80$, then the sum in (8.6) is taken over $j \leq j_{1}+C$ and is dominated by

$$
C 2^{k} \sum_{j \leq j_{1}+C} 2^{-j_{1} / 2} 2^{-\max \left(j, j_{2}\right) / 4} \leq C\left(j_{2}+1\right) 2^{-j_{2} / 4},
$$

which suffices. The case $j_{2}=\max \left(j_{1}, j_{2}\right) \geq 2 k-80$ is identical. This completes the proof of 8.3 .

We turn to the proof of (8.1). We use the representation (4.1). If $f_{k_{1}}=f_{k_{1}, j_{1}} \in$ $X_{k_{1}}$ and $f_{k_{2}}=f_{k_{2}, j_{2}} \in X_{k_{2}}$, then (8.1) follows directly from (8.3) and the definitions. Assume now that $f_{k_{1}}=g_{k_{1}} \in Y_{k_{1}}, f_{k_{2}}=g_{k_{2}} \in Y_{k_{2}},\left\|f_{k_{1}}\right\|_{Z_{k_{1}}} \approx\left\|g_{k_{1}}\right\|_{Y_{k_{1}}}$, and $\left\|f_{k_{2}}\right\|_{Z_{k_{2}}} \approx\left\|g_{k_{2}}\right\|_{Y_{k_{2}}}$. For $j_{1} \in\left[0, k_{1}\right]$ and $j_{2} \in\left[0, k_{2}\right]$ let

$$
g_{k_{1}, j_{1}}(\xi, \tau)=\eta_{j_{1}}(\tau-\omega(\xi)) g_{k_{1}}(\xi, \tau) \quad \text { and } \quad g_{k_{2}, j_{2}}(\xi, \tau)=\eta_{j_{2}}(\tau-\omega(\xi)) g_{k_{2}}(\xi, \tau) .
$$


We use (8.3), Lemma (4.1)(b), and the definition (8.4) in the case $\max \left(j_{1}, j_{2}\right) \leq$ $2 k-80$ to write

$$
\begin{aligned}
& 2^{k}\left\|\eta_{k}(\xi) \cdot(\tau-\omega(\xi)+i)^{-1} g_{k_{1}} * g_{k_{2}}\right\|_{X_{k}} \\
& \quad \leq C \sum_{j_{1}, j_{2} \leq k+30} 2^{k}\left\|\eta_{k}(\xi) \cdot(\tau-\omega(\xi)+i)^{-1} g_{k_{1}, j_{1}} * g_{k_{2}, j_{2}}\right\|_{X_{k}} \\
& \quad \leq C \sum_{j_{1}, j_{2} \leq k+30} \gamma\left(j_{1}, j_{2}, k\right) 2^{j_{1} / 2}\left\|g_{k_{1}, j_{1}}\right\|_{L^{2}} 2^{j_{2} / 2}\left\|g_{k_{2}, j_{2}}\right\|_{L^{2}} \\
& \quad \leq C\left\|g_{k_{1}}\right\|_{Y_{k_{1}}}\left\|g_{k_{2}}\right\|_{Y_{k_{2}}},
\end{aligned}
$$

as desired.

Finally, assume $f_{k_{1}}=f_{k_{1}, j_{1}} \in X_{k_{1}}, f_{k_{2}}=g_{k_{2}} \in Y_{k_{2}},\left\|f_{k_{2}}\right\|_{Z_{k_{2}}} \approx\left\|g_{k_{2}}\right\|_{Y_{k_{2}}}$, and $\left\|f_{k_{1}}\right\|_{Z_{k_{1}}} \approx 2^{j_{1} / 2} \beta_{k_{1}, j_{1}}|| f_{k_{1}, j_{1}} \|_{L^{2}}$, and write $g_{k_{2}}=\sum_{j_{2}=0}^{k_{2}} g_{k_{2}, j_{2}}$ as before. If $j_{1} \leq 2 k-80$, then we can use the same computation as before. If $j_{1} \geq 2 k-80$, then we use (8.3), Lemma (4.1) (b), and the definition (8.4) to write

$$
\begin{aligned}
& 2^{k}\left\|\eta_{k}(\xi) \cdot(\tau-\omega(\xi)+i)^{-1} f_{k_{1}, j_{1}} * g_{k_{2}}\right\|_{X_{k}} \\
& \quad \leq C \sum_{j_{2} \leq k_{2}} 2^{k}\left\|\eta_{k}(\xi) \cdot(\tau-\omega(\xi)+i)^{-1} f_{k_{1}, j_{1}} * g_{k_{2}, j_{2}}\right\|_{X_{k}} \\
& \quad \leq C \sum_{j_{2} \leq k_{2}} 2^{-j_{2} / 8} 2^{j_{1} / 2} \beta_{k_{1}, j_{1}}\left\|f_{k_{1}, j_{1}}\right\|_{L^{2}} 2^{j_{2} / 2}\left\|g_{k_{2}, j_{2}}\right\|_{L^{2}} \\
& \quad \leq C 2^{j_{1} / 2} \beta_{k_{1}, j_{1}}\left\|f_{k_{1}, j_{1}}\right\|_{L^{2}}\left\|g_{k_{2}}\right\|_{Y_{k_{2}}},
\end{aligned}
$$

as desired. This completes the proof of (8.1) in the case $\min \left(k, k_{1}, k_{2}\right) \geq 200$.

Case 2: $\min \left(k, k_{1}, k_{2}\right) \leq 200$. In view of the hypothesis, $\max \left(k, k_{1}, k_{2}\right) \leq$ 230. If $k_{1}=0$ or $k_{2}=0$, we may replace the spaces $Z_{0}$ in the right-hand of (8.1) with the larger spaces $\bar{Z}_{0}$; see the definition (3.8). Clearly, the proofs are identical to the proofs in the corresponding cases $k_{1}=1$ or $k_{2}=1$. Therefore we may assume $k_{1}, k_{2} \geq 1$. In view of Lemma 4.1 (b) and the representation (4.1), we may assume $f_{k_{1}}=f_{k_{1}, j_{1}}$ is supported in $D_{k_{1}, j_{1}}, f_{k_{2}}=f_{k_{2}, j_{2}}$ is supported in $D_{k_{2}, j_{2}},\left\|f_{k_{1}}\right\|_{Z_{k_{1}}} \approx 2^{j_{1} / 2} \beta_{k_{1}, j_{1}}\left\|f_{k_{1}, j_{1}}\right\|_{L^{2}} \approx 2^{j_{1}}|| f_{k_{1}, j_{1}} \|_{L^{2}}$, and $\left\|\left.f_{k_{2}}\right|_{Z_{k_{2}}} \approx 2^{j_{2} / 2} \beta_{k_{2}, j_{2}}|| f_{k_{2}, j_{2}}\right\|_{L^{2}} \approx 2^{j_{2}}\left\|f_{k_{2}, j_{2}}\right\|_{L^{2}}$. Using the definitions and the fact that $k \leq 230$, for (8.1) it suffices to prove that

$$
\begin{aligned}
\sum_{j} 2^{j} \| \mathcal{F}^{-1}\left[\eta_{j}(\tau) \eta_{k}(\xi)(\tau+i)^{-1}\right. & \left.f_{k_{1}, j_{1}} * f_{k_{2}, j_{2}}\right]||_{L_{x}^{1} L_{t}^{2}} \\
& \leq C 2^{j_{1}}|| f_{k_{1}, j_{1}}\left\|_{L^{2}} \cdot 2^{j_{2}}\right\| f_{k_{2}, j_{2}} \|_{L^{2}} .
\end{aligned}
$$

By examining the supports of the functions, we may assume that the sum in (8.7) is taken over

$$
j \leq \max \left(j_{1}, j_{2}\right)+C .
$$

Assume $j_{1}=\max \left(j_{1}, j_{2}\right)$ (the case $j_{2}=\max \left(j_{1}, j_{2}\right)$ is identical). The left-hand side of (8.7) is dominated by

$$
\begin{aligned}
C \sum_{j \leq j_{1}+C}\left\|\mathcal{F}^{-1}\left(f_{k_{1}, j_{1}} * f_{k_{2}, j_{2}}\right)\right\|_{L_{x}^{1} L_{t}^{2}} & \leq\left(j_{1}+C\right)\left\|\mathcal{F}^{-1}\left(f_{k_{1}, j_{1}}\right)\right\|_{L^{2}}\left\|\mathcal{F}^{-1}\left(f_{k_{2}, j_{2}}\right)\right\|_{L_{x}^{2} L_{t}^{\infty}} \\
& \leq C 2^{j_{1}}\left\|f_{k_{1}, j_{1}}\right\|_{L^{2}} \cdot 2^{j_{2} / 2}\left\|f_{k_{2}, j_{2}}\right\|_{L^{2}},
\end{aligned}
$$

which completes the proof of (8.7). 
For later use, we rewrite the stronger bound that we proved in this last case: if $k, k_{1}, k_{2} \in \mathbb{Z}_{+}$have the property that $\max \left(k, k_{1}, k_{2}\right) \leq \min \left(k, k_{1}, k_{2}\right)+30 \leq 230$, $f_{k_{1}} \in \bar{Z}_{k_{1}}$, and $f_{k_{2}} \in \bar{Z}_{k_{2}}$, then

$$
2^{k}\left\|\eta_{k}(\xi) \cdot A_{k}(\xi, \tau)^{-1} f_{k_{1}} * f_{k_{2}}\right\|_{Z_{k}} \leq C\left\|f_{k_{1}}\right\|_{\bar{Z}_{k_{1}}}\left\|f_{k_{2}}\right\|_{\bar{Z}_{k_{2}}}
$$

where $\bar{Z}_{k}=Z_{k}$ if $k \geq 1$ and $\bar{Z}_{k}=\bar{Z}_{0}$ if $k=0$.

Proof of Proposition 8.2. We analyze two cases.

Case 1: $k \geq 1$. We show first that if $j_{1}, j_{2} \geq 0, f_{k_{1}, j_{1}}$ is an $L^{2}$ function supported in $D_{k_{1}, j_{1}}$, and $f_{k_{2}, j_{2}}$ is an $L^{2}$ function supported in $D_{k_{2}, j_{2}}$, then

$$
\begin{array}{r}
2^{k} \sum_{j} 2^{j / 2} \beta_{k, j}\left\|\eta_{k}(\xi) \eta_{j}(\tau-\omega(\xi))(\tau-\omega(\xi)+i)^{-1}\left(f_{k_{1}, j_{1}} * f_{k_{2}, j_{2}}\right)\right\|_{L^{2}} \\
\leq C \gamma^{\prime}\left(j_{1}, j_{2}, k\right) 2^{j_{1} / 2} \beta_{k_{1}, j_{1}}\left\|f_{k_{1}, j_{1}}\right\|_{L^{2}} \cdot 2^{j_{2} / 2} \beta_{k_{2}, j_{2}}\left\|f_{k_{2}, j_{2}}\right\|_{L^{2}},
\end{array}
$$

where

$$
\gamma^{\prime}\left(j_{1}, j_{2}, k\right)=\left(2^{k / 2}+2^{\max \left(j_{1}, j_{2}\right) / 4}\right)^{-2 / 3} .
$$

To prove (8.10), we notice that, in view of (6.23),

$$
\eta_{k}(\xi) \eta_{j}(\tau-\omega(\xi))(\tau-\omega(\xi)+i)^{-1}\left(f_{k_{1}, j_{1}} * f_{k_{2}, j_{2}}\right) \equiv 0
$$

unless

$$
\left\{\begin{array}{l}
\max \left(j, j_{1}, j_{2}\right) \in\left[k+k_{1}-10, k+k_{1}+10\right] \text { or } \\
\max \left(j, j_{1}, j_{2}\right) \geq k+k_{1}+10 \text { and } \max \left(j, j_{1}, j_{2}\right)-\operatorname{med}\left(j, j_{1}, j_{2}\right) \leq 10
\end{array}\right.
$$

Also, combining (6.20) and (6.21),

$$
\begin{aligned}
& \left\|\eta_{k}(\xi) \eta_{j}(\tau-\omega(\xi))(\tau-\omega(\xi)+i)^{-1}\left(f_{k_{1}, j_{1}} * f_{k_{2}, j_{2}}\right)\right\|_{L^{2}} \leq C 2^{-j} 2^{\left(j+j_{1}+j_{2}\right) / 2} \\
& \quad \times\left[2^{(j+k) / 2}+2^{\left(\max \left(j_{1}, j_{2}\right)+k_{1}\right) / 2}+2^{\max \left(j, j_{1}, j_{2}\right) / 2} 2^{\operatorname{med}\left(j, j_{1}, j_{2}\right) / 4}\right]^{-1}\left\|f_{k_{1}, j_{1}}\right\|_{L^{2}}\left\|f_{k_{2}, j_{2}}\right\|_{L^{2}} .
\end{aligned}
$$

Thus, for (8.10), it suffices to prove that

$$
\begin{aligned}
2^{k} \sum_{j} \beta_{k, j}\left[2^{(j+k) / 2}+2^{\left(\max \left(j_{1}, j_{2}\right)+k_{1}\right) / 2}+2^{\max \left(j, j_{1}, j_{2}\right) / 2} 2^{\operatorname{med}\left(j, j_{1}, j_{2}\right) / 4}\right]^{-1} \\
\leq C \gamma^{\prime}\left(j_{1}, j_{2}, k\right) \beta_{k_{1}, j_{1}} \beta_{k_{2}, j_{2}},
\end{aligned}
$$

where the sum in (8.13) is taken over $j$ satisfying (8.12). If $\max \left(j_{1}, j_{2}\right) \leq k+k_{1}-20$, then $j \in\left[k+k_{1}-10, k+k_{1}+10\right]$; we ignore the term $2^{\left(\max \left(j_{1}, j_{2}\right)+k_{1}\right) / 2}$ and the bound (8.13) follows easily from the definitions. If $j_{1}=\max \left(j_{1}, j_{2}\right) \geq k+k_{1}-20$, then the sum in (8.13) is taken over $j \leq j_{1}+C$. The left-hand side of (8.13) is dominated by

$$
C 2^{k} \sum_{j \leq j_{1}+C} \beta_{k, j} 2^{-\left(\max \left(j_{1}, j_{2}\right)+k_{1}\right) / 2} \leq C k_{1} 2^{-k_{1} / 2} \leq C \gamma^{\prime}\left(j_{1}, j_{2}, k\right) \beta_{k_{1}, j_{1}} .
$$

The case $j_{2}=\max \left(j_{1}, j_{2}\right) \geq k+k_{1}-20$ is identical, which completes the proof of (8.10).

We turn to the proof of (8.2). We use the representation (4.1). If $f_{k_{1}}=f_{k_{1}, j_{1}} \in$ $X_{k_{1}}$ and $f_{k_{2}}=f_{k_{2}, j_{2}} \in X_{k_{2}}$, then (8.2) follows directly from (8.10) and the definitions. Assume now that $f_{k_{1}}=g_{k_{1}} \in Y_{k_{1}}, f_{k_{2}}=g_{k_{2}} \in Y_{k_{2}},\left\|f_{k_{1}}\right\|_{Z_{k_{1}}} \approx\left\|g_{k_{1}}\right\|_{Y_{k_{1}}}$, and $\left\|f_{k_{2}}\right\|_{Z_{k_{2}}} \approx\left\|g_{k_{2}}\right\|_{Y_{k_{2}}}$. For $j_{1} \in\left[0, k_{1}\right]$ and $j_{2} \in\left[0, k_{2}\right]$ let $g_{k_{1}, j_{1}}(\xi, \tau)=$ 
$\eta_{j_{1}}(\tau-\omega(\xi)) g_{k_{1}}(\xi, \tau)$ and $g_{k_{2}, j_{2}}(\xi, \tau)=\eta_{j_{2}}(\tau-\omega(\xi)) g_{k_{2}}(\xi, \tau)$. We use (8.10) and Lemma (4.1) (b) to write

$$
\begin{aligned}
& 2^{k}\left\|\eta_{k}(\xi) \cdot(\tau-\omega(\xi)+i)^{-1} g_{k_{1}} * g_{k_{2}}\right\|_{X_{k}} \\
& \quad \leq C \sum_{j_{1}, j_{2} \leq k_{1}+10} 2^{k}\left\|\eta_{k}(\xi) \cdot(\tau-\omega(\xi)+i)^{-1} g_{k_{1}, j_{1}} * g_{k_{2}, j_{2}}\right\|_{X_{k}} \\
& \quad \leq C \sum_{j_{1}, j_{2} \leq k_{1}+10} \gamma^{\prime}\left(j_{1}, j_{2}, k\right) 2^{j_{1} / 2}\left\|g_{k_{1}, j_{1}}\right\|_{L^{2}} 2^{j_{2} / 2}\left\|g_{k_{2}, j_{2}}\right\|_{L^{2}} \\
& \quad \leq\left. C 2^{-k / 4}\left\|g_{k_{1}}\right\|\right|_{Y_{k_{1}}}\left\|g_{k_{2}}\right\|_{Y_{k_{2}}},
\end{aligned}
$$

as desired. Finally, if $f_{k_{1}}=f_{k_{1}, j_{1}} \in X_{k_{1}}, f_{k_{2}}=g_{k_{2}} \in Y_{k_{2}},\left\|f_{k_{2}}\right\|_{Z_{k_{2}}} \approx\left\|g_{k_{2}}\right\|_{Y_{k_{2}}}$, and $\left\|f_{k_{1}}\right\|_{Z_{k_{1}}} \approx 2^{j_{1} / 2} \beta_{k_{1}, j_{1}}\left\|f_{k_{1}, j_{1}}\right\|_{L^{2}}$, we write $g_{k_{2}}=\sum_{j_{2}=0}^{k_{2}} g_{k_{2}, j_{2}}$ as before and repeat the same estimate, without the sum in $j_{1}$. This completes the proof of (8.2) in the case $k \geq 1$.

Case 2: $k=0$. We show first that if $j_{1}, j_{2} \geq 0, f_{k_{1}, j_{1}}$ is an $L^{2}$ function supported in $D_{k_{1}, j_{1}}$, and $f_{k_{2}, j_{2}}$ is an $L^{2}$ function supported in $D_{k_{2}, j_{2}}$, then

$$
\begin{aligned}
& \sum_{k^{\prime}=-\infty}^{1} \sum_{j=0}^{\infty} 2^{j-k^{\prime}}\left\|\chi_{k^{\prime}}(\xi) \eta_{j}(\tau) \cdot \xi(\tau+i)^{-1}\left(f_{k_{1}, j_{1}} * f_{k_{2}, j_{2}}\right)\right\|_{L^{2}} \\
& \quad \leq C 2^{-\max \left(j_{1}, j_{2}\right) / 4} \cdot 2^{j_{1} / 2} \beta_{k_{1}, j_{1}}\left\|f_{k_{1}, j_{1}}\right\|_{L^{2}} \cdot 2^{j_{2} / 2} \beta_{k_{2}, j_{2}}\left\|f_{k_{2}, j_{2}}\right\|_{L^{2}} .
\end{aligned}
$$

To prove (8.14), we notice that, in view of (6.23),

$$
\chi_{k^{\prime}}(\xi) \eta_{j}(\tau) \cdot \xi(\tau+i)^{-1}\left(f_{k_{1}, j_{1}} * f_{k_{2}, j_{2}}\right) \equiv 0
$$

unless

$$
\left\{\begin{array}{l}
\max \left(j, j_{1}, j_{2}\right) \in\left[k^{\prime}+k_{1}-10, k^{\prime}+k_{1}+10\right] \text { or } \\
\max \left(j, j_{1}, j_{2}\right) \geq k^{\prime}+k_{1}+10 \text { and } \max \left(j, j_{1}, j_{2}\right)-\operatorname{med}\left(j, j_{1}, j_{2}\right) \leq 10
\end{array}\right.
$$

Also, using (6.19),

$$
\begin{aligned}
& \left\|\chi_{k^{\prime}}(\xi) \eta_{j}(\tau) \cdot \xi(\tau+i)^{-1}\left(f_{k_{1}, j_{1}} * f_{k_{2}, j_{2}}\right)\right\|_{L^{2}} \\
& \quad \leq C 2^{k^{\prime}-j} 2^{k^{\prime} / 2} 2^{\left(j_{1}+j_{2}\right) / 2} 2^{-\max \left(j_{1}, j_{2}\right) / 2}\left\|f_{k_{1}, j_{1}}\right\|_{L^{2}}\left\|f_{k_{2}, j_{2}}\right\|_{L^{2}} .
\end{aligned}
$$

Thus, for (8.14), it suffices to prove that

$$
\sum_{k^{\prime}=-\infty}^{1} \sum_{j} 2^{k^{\prime} / 2} \leq C 2^{\max \left(j_{1}, j_{2}\right) / 4}
$$

where the sum in (8.16) is taken over $j$ satisfying (8.15). If $\max \left(j_{1}, j_{2}\right) \leq k^{\prime}+k_{1}-20$, then $j \in\left[k^{\prime}+k_{1}-10, k^{\prime}+k_{1}+10\right]$, so (8.16) is clear. If $\max \left(j_{1}, j_{2}\right) \geq k^{\prime}+k_{1}-20$, then the sum in (8.16) is taken over $j \leq \max \left(j_{1}, j_{2}\right)+C$, and (8.16) follows easily.

Given (8.14), the bound (8.2) follows as in the case $k \geq 1$, using the definition of the space $X_{0}$. This completes the proof of Proposition 8.2.

For later use, we notice that the bound (8.14) also shows that

$$
\left\|\eta_{0}(\xi) \cdot(\tau+i)^{-1} f_{k_{1}} * f_{k_{2}}\right\|_{\bar{Z}_{0}} \leq C|| f_{k_{1}}\left\|_{Z_{k_{1}}}\right\| f_{k_{2}} \|_{Z_{k_{2}}} .
$$




\section{Multiplication By SMOOTh Bounded FunCtions}

In this section we consider operators on $Z_{k}$ given by convolutions with Fourier transforms of certain smooth bounded functions. For integers $N \geq 100$ we define the space of admissible factors

$$
\begin{aligned}
& S_{N}^{\infty}=\left\{m: \mathbb{R}^{2} \rightarrow \mathbb{C}: m \text { is supported in } \mathbb{R} \times[-2,2]\right. \text { and } \\
& \left.\|m\|_{S_{N}^{\infty}}:=\sum_{\sigma_{1}=0}^{N}\left\|\partial_{t}^{\sigma_{1}} m\right\|_{L_{x, t}^{\infty}}+\sum_{\sigma_{1}=0}^{N} \sum_{\sigma_{2}=1}^{N}\left\|\partial_{t}^{\sigma_{1}} \partial_{x}^{\sigma_{2}} m\right\|_{L_{x, t}^{2}}<\infty\right\} .
\end{aligned}
$$

The precise value of $N$ is not important (in fact, we will always take $N=100$ or $N=110$ ). Notice that bounded functions such as $\psi(t) e^{i q U_{0}}, q \in \mathbb{R}, U_{0}$ as in (2.9), are in $S_{N}^{\infty}$. We also define the space of restricted admissible factors

$$
\begin{array}{r}
S_{N}^{2}=\left\{m: \mathbb{R}^{2} \rightarrow \mathbb{C}: m \text { is supported in } \mathbb{R} \times[-2,2]\right. \text { and } \\
\left.\|m\|_{S_{N}^{2}}:=\sum_{\sigma_{1}=0}^{N} \sum_{\sigma_{2}=0}^{N}\left\|\partial_{t}^{\sigma_{1}} \partial_{x}^{\sigma_{2}} m\right\|_{L_{x, t}^{2}}<\infty\right\} .
\end{array}
$$

Using the Sobolev imbedding theorem, it is easy to verify the following properties:

$$
\left\{\begin{array}{l}
S_{N}^{2} \subseteq S_{N-10}^{\infty} \\
S_{N}^{\infty} \cdot S_{N}^{\infty} \subseteq S_{N-10}^{\infty} \\
S_{N}^{2} \cdot S_{N}^{\infty} \subseteq S_{N-10}^{2} \\
\partial_{x} S_{N}^{\infty} \subseteq S_{N-10}^{2}
\end{array}\right.
$$

For $k \in \mathbb{Z}_{+}$we define

$$
Z_{k}^{\text {high }}=\left\{f_{k} \in Z_{k}: f_{k} \text { is supported in }\left\{\tau-\omega(\xi) \in \bigcup_{j \geq k-20} \widetilde{I}_{j}\right\}\right\} .
$$

Clearly, $Z_{k}^{\text {high }}=Z_{k}$ if $k \leq 20$. For $k \in \mathbb{Z}_{+}$and $\epsilon \in\{-1,0\}$ let $A_{k}^{\epsilon}(\xi, \tau)=\left[A_{k}(\xi, \tau)\right]^{\epsilon}$.

Lemma 9.1. Assume $k_{1}, k_{2} \in \mathbb{Z}_{+},\left|k_{1}-k_{2}\right| \leq 10$, and $f_{k_{1}}^{\text {high }} \in Z_{k_{1}}^{\text {high }}$. Then, for $m \in S_{100}^{\infty}$ and $\epsilon \in\{-1,0\}$,

$$
\left\|\eta_{k_{2}}\left(\xi_{2}\right) A_{k_{2}}^{\epsilon}\left(\xi_{2}, \tau_{2}\right) \cdot \mathcal{F}\left[m \cdot \mathcal{F}^{-1}\left(f_{k_{1}}^{\text {high }}\right)\right]\left(\xi_{2}, \tau_{2}\right)\right\|_{Z_{k_{2}}} \leq C\|m\|_{S_{100}^{\infty}} \cdot\left\|A_{k_{1}}^{\epsilon} f_{k_{1}}^{\text {high }}\right\|_{Z_{k_{1}}} .
$$

Remark. We do not need to consider convolutions of low-modulation functions and Fourier transforms of admissible factors, in view of the identity (10.13).

Proof of Lemma 9.1. We may assume $\|m\|_{S_{100}^{\infty}}=1$. For any $j^{\prime \prime} \in \mathbb{Z}_{+}$and $k^{\prime \prime} \in \mathbb{Z}$ let

$$
m_{k^{\prime \prime}, j^{\prime \prime}}=\mathcal{F}^{-1}\left[\eta_{j^{\prime \prime}}(\tau) \chi_{k^{\prime \prime}}(\xi) \mathcal{F}(m)\right]
$$

and $m_{\leq k^{\prime \prime}, j^{\prime \prime}}=\sum_{k^{\prime \prime \prime} \leq k^{\prime \prime}} m_{k^{\prime \prime \prime}, j^{\prime \prime}}$. Using (9.1) and the Sobolev imbedding theorem,

$$
\left\|\partial_{t}^{\sigma_{1}} \partial_{x}^{\sigma_{2}} m\right\|_{L_{x}^{2} L_{t}^{\infty}} \leq C \text { for any } \sigma_{1} \in \mathbb{Z} \cap[0,90], \sigma_{2} \in \mathbb{Z} \cap[1,90] .
$$

Thus, for any $j^{\prime \prime} \in \mathbb{Z}_{+}$and $k^{\prime \prime} \in \mathbb{Z}$,

$$
\left\{\begin{array}{l}
\left\|m_{\leq k^{\prime \prime}, j^{\prime \prime}}\right\| \|_{L_{x, t}^{\infty} \leq C 2^{-80 j^{\prime \prime}}} ; \\
2^{k^{\prime \prime}}\left\|m_{k^{\prime \prime}, j^{\prime \prime}}\right\|_{L_{x}^{2} L_{t}^{\infty}}+\left\|m_{k^{\prime \prime}, j^{\prime \prime}}\right\|_{L_{x, t}^{\infty}} \leq C\left(1+2^{k^{\prime \prime}}\right)^{-80} 2^{-80 j^{\prime \prime}} .
\end{array}\right.
$$


We turn now to the proof of (9.5). Assume first that $k_{1}, k_{2} \geq 1$. In view of the definition of $Z_{k}^{\text {high }}$ and Lemma 4.1(b), we may assume that $f_{k_{1}}^{\text {high }}=f_{k_{1}, j_{1}}$ is an $L^{2}$ function supported in $D_{k_{1}, j_{1}}, j_{1} \geq k_{1}-20,\left\|A_{k_{1}}^{\epsilon} f_{k_{1}}^{\text {high }}\right\|_{Z_{k_{1}}} \approx 2^{\epsilon j_{1}} 2^{j_{1} / 2} \beta_{k_{1}, j_{1}}\left\|f_{k_{1}, j_{1}}\right\|_{L^{2}}$. We write

$$
m=\sum_{j^{\prime \prime}=0}^{\infty} m_{\leq-100, j^{\prime \prime}}+\sum_{k^{\prime \prime}=-99}^{\infty} \sum_{j^{\prime \prime}=0}^{\infty} m_{k^{\prime \prime}, j^{\prime \prime}} .
$$

For (9.5) it suffices to prove that for $\epsilon \in\{-1,0\}$

$$
\begin{aligned}
& \sum_{j^{\prime \prime} \geq 0}\left\|\eta_{k_{2}}\left(\xi_{2}\right) A^{\epsilon}\left(\xi_{2}, \tau_{2}\right) \cdot\left[f_{k_{1}, j_{1}} * \mathcal{F}\left(m_{\leq-100, j^{\prime \prime}}\right)\right]\left(\xi_{2}, \tau_{2}\right)\right\|_{Z_{k_{2}}} \\
& +\sum_{k^{\prime \prime} \geq-99} \sum_{j^{\prime \prime} \geq 0}\left\|\eta_{k_{2}}\left(\xi_{2}\right) A^{\epsilon}\left(\xi_{2}, \tau_{2}\right) \cdot\left[f_{k_{1}, j_{1}} * \mathcal{F}\left(m_{k^{\prime \prime}, j^{\prime \prime}}\right)\right]\left(\xi_{2}, \tau_{2}\right)\right\|_{Z_{k_{2}}} \\
& \leq C 2^{\epsilon j_{1}} \cdot 2^{j_{1} / 2} \beta_{k_{1}, j_{1}}\left\|f_{k_{1}, j_{1}}\right\|_{L^{2}} .
\end{aligned}
$$

To bound the first sum in (9.9), we make the changes of variables $\tau_{2}=\mu_{2}+\omega\left(\xi_{2}\right)$, $\tau_{1}=\mu_{1}+\omega\left(\xi_{1}\right)$ and write

$$
\begin{aligned}
& f_{k_{1}, j_{1}} * \mathcal{F}\left(m_{\leq-100, j^{\prime \prime}}\right)\left(\xi_{2}, \mu_{2}+\omega\left(\xi_{2}\right)\right) \\
& =\int_{\mathbb{R}^{2}} f_{k_{1}, j_{1}}\left(\xi_{1}, \mu_{1}+\omega\left(\xi_{1}\right)\right) \mathcal{F}\left(m_{\leq-100, j^{\prime \prime}}\right)\left(\xi_{2}-\xi_{1}, \mu_{2}-\mu_{1}+\omega\left(\xi_{2}\right)-\omega\left(\xi_{1}\right)\right) d \xi_{1} d \tau_{1} .
\end{aligned}
$$

By examining the supports of the functions and using the fact that $\left|\omega\left(\xi_{2}\right)-\omega\left(\xi_{1}\right)\right| \leq$ $2^{k_{1}-50}$ if $\left|\xi_{2}-\xi_{1}\right| \leq 2^{-99}$, together with $j_{1} \geq k_{1}-20$, we see that $\eta_{j_{2}}\left(\tau_{2}-\omega\left(\xi_{2}\right)\right)$. $\left[f_{k_{1}, j_{1}} * \mathcal{F}\left(m_{\leq-100, j^{\prime \prime}}\right)\right]\left(\xi_{2}, \tau_{2}\right) \equiv 0$ unless

$$
\left|j_{1}-j_{2}\right| \leq C \text { or } j_{1}, j_{2} \leq j^{\prime \prime}+C \text {. }
$$

We use the $X_{k_{2}}$ norm to bound the first sum in (9.9). Using Plancherel's theorem and (9.7),

$$
\left\|f_{k_{1}, j_{1}} * \mathcal{F}\left(m_{\leq-100, j^{\prime \prime}}\right)\right\|_{L_{\xi_{2}, \tau_{2}}^{2}} \leq C 2^{-80 j^{\prime \prime}}\left\|f_{k_{1}, j_{1}}\right\|_{L^{2}} .
$$

Thus, the $X_{k_{2}}$ norm of the first sum in (9.9) is dominated by

$$
C \sum_{j^{\prime \prime} \geq 0} \sum_{j_{2} \geq 0} 2^{\epsilon j_{2}} 2^{j_{2} / 2} \beta_{k_{2}, j_{2}} 2^{-80 j^{\prime \prime}}\left\|f_{k_{1}, j_{1}}\right\|_{L^{2}}
$$

where the sum is over $j_{2}, j^{\prime \prime}$ satisfying (9.10). The bound (9.9) for the first sum follows easily (recall that $\left|k_{1}-k_{2}\right| \leq 10$ ).

To bound the second sum in (9.9), assume first that $\epsilon=0$. We notice that if $\left|\xi_{2}-\xi_{1}\right| \in\left[2^{k^{\prime \prime}-1}, 2^{k^{\prime \prime}+1}\right]$, then $\left|\omega\left(\xi_{2}\right)-\omega\left(\xi_{1}\right)\right| \leq C 2^{k_{1}+k^{\prime \prime}}$, so

$$
\eta_{j_{2}}\left(\tau_{2}-\omega\left(\xi_{2}\right)\right) \cdot\left[f_{k_{1}, j_{1}} * \mathcal{F}\left(m_{k^{\prime \prime}, j^{\prime \prime}}\right)\right]\left(\xi_{2}, \tau_{2}\right) \equiv 0
$$

unless

$$
\left|j_{1}-j_{2}\right| \leq 4 \text { or } j_{1}, j_{2} \leq k_{1}+k^{\prime \prime}+j^{\prime \prime}+C .
$$

Using Plancherel's theorem and (9.7),

$$
\left\|f_{k_{1}, j_{1}} * \mathcal{F}\left(m_{k^{\prime \prime}, j^{\prime \prime}}\right)\right\|_{L_{\xi_{2}, \tau_{2}}^{2}} \leq C 2^{-80 k^{\prime \prime}} 2^{-80 j^{\prime \prime}}\left\|f_{k_{1}, j_{1}}\right\|_{L^{2}}
$$

The bound (9.9) for the second sum follows by using the $X_{k_{2}}$ norm since

$$
\sum_{j_{2} \leq j_{1}+k^{\prime \prime}+j^{\prime \prime}+C} 2^{j_{2} / 2} \beta_{k_{2}, j_{2}} \leq C 2^{10 k^{\prime \prime}} 2^{10 j^{\prime \prime}} \cdot 2^{j_{1} / 2} \beta_{k_{1}, j_{1}} .
$$


We bound now the second sum in (9.9) when $\epsilon=-1$. The main difficulty is the presence of the indices $j_{2} \ll j_{1}$. In fact, for indices $j_{2} \geq j_{1}-10$, the argument above applies since the left-hand side is multiplied by $2^{-j_{2}}$ and the right-hand side is multiplied by $2^{-j_{1}}$. In view of (9.11), it suffices to prove that

$$
\begin{aligned}
& \sum_{k^{\prime \prime}+j^{\prime \prime} \geq j_{1}-k_{1}-C}\left\|\eta_{k_{2}}\left(\xi_{2}\right) \eta_{\leq k_{2}-1}\left(\tau_{2}-\omega\left(\xi_{2}\right)\right) A_{k_{2}}^{-1}\left(\xi_{2}, \tau_{2}\right)\left[f_{k_{1}, j_{1}} * \mathcal{F}\left(m_{k^{\prime \prime}, j^{\prime \prime}}\right)\right]\left(\xi_{2}, \tau_{2}\right)\right\|_{Y_{k_{2}}} \\
& +\sum_{k^{\prime \prime}+j^{\prime \prime} \geq j_{1}-k_{1}-C} \sum_{j_{2} \geq k_{2}} \sum^{j_{2} \leq j_{1}-10} 2^{-j_{2} / 2} \beta_{k_{2}, j_{2}}\left\|\eta_{k_{2}}\left(\xi_{2}\right) \eta_{j_{2}}\left(\tau_{2}-\omega\left(\xi_{2}\right)\right) f_{k_{1}, j_{1}} * \mathcal{F}\left(m_{k^{\prime \prime}, j^{\prime \prime}}\right)\right\|_{L^{2}} \\
& \leq C 2^{-j_{1} / 2} \beta_{k_{1}, j_{1}}\left\|f_{k_{1}, j_{1}}\right\|_{L^{2}} .
\end{aligned}
$$

Using Lemma 4.1(c) and (9.7), the first sum in the left-hand side of (9.13) is dominated by

$$
C \sum_{k^{\prime \prime}+j^{\prime \prime} \geq j_{1}-k_{1}-C} 2^{-k_{2} / 2}\left\|f_{k_{1}, j_{1}}\right\|_{L^{2}} \cdot\left\|m_{k^{\prime \prime}, j^{\prime \prime}}\right\|_{L_{x}^{2} L_{t}^{\infty}} \leq C 2^{-k_{2} / 2}\left\|f_{k_{1}, j_{1}}\right\|_{L^{2} \cdot 2^{-70\left(j_{1}-k_{1}\right)},}
$$

which clearly suffices. Using (9.12), the second sum in the left-hand side of (9.13) is dominated by

$$
C 2^{-70\left(j_{1}-k_{1}\right)}\left\|f_{k_{1}, j_{1}}\right\|_{L^{2}} \cdot \sup _{j_{2} \in\left[k_{2}, j_{1}\right]} 2^{-j_{2} / 2} \beta_{k_{2}, j_{2}} \leq C 2^{-j_{1} / 2}\left\|f_{k_{1}, j_{1}}\right\|_{L^{2}},
$$

which completes the proof of (9.13).

We prove now the bound (9.5) in the case $k_{1}=k_{2}=0$. We use the representation (4.2). Assume first that $f_{0}^{\text {high }}=g_{0, j_{1}}$ is an $L^{2}$ function supported in $\widetilde{I}_{0} \times \widetilde{I}_{j_{1}}$, $\left\|A_{0}^{\epsilon} f_{0}^{\text {high }}\right\|_{Z_{0}} \approx 2^{\epsilon j_{1}} 2^{j_{1}}\left\|\mathcal{F}^{-1}\left(g_{0, j_{1}}\right)\right\|_{L_{x}^{1} L_{t}^{2}}$. We write

$$
m=\sum_{j^{\prime \prime}=0}^{\infty} m_{\leq 4, j^{\prime \prime}}+\sum_{k^{\prime \prime}=5}^{\infty} \sum_{j^{\prime \prime}=0}^{\infty} m_{k^{\prime \prime}, j^{\prime \prime}}
$$

and notice that $\eta_{0}\left(\xi_{2}\right)\left(g_{0, j_{1}} * \mathcal{F}\left(m_{k^{\prime \prime}, j^{\prime \prime}}\right)\right)\left(\xi_{2}, \tau_{2}\right) \equiv 0$ if $k^{\prime \prime} \geq 5$. For (9.5), using only the $Y_{0}$ norm, it suffices to prove that for $\epsilon \in\{-1,0\}$

$$
\begin{gathered}
\sum_{j^{\prime \prime}=0}^{\infty} \sum_{j_{2}=0}^{\infty} 2^{\epsilon j_{2}} 2^{j_{2}}\left\|\mathcal{F}^{-1}\left[\eta_{j_{2}}\left(\tau_{2}\right)\left(g_{0, j_{1}} * \mathcal{F}\left(m_{\leq 4, j^{\prime \prime}}\right)\right)\left(\xi_{2}, \tau_{2}\right)\right]\right\|_{L_{x}^{1} L_{t}^{2}} \\
\leq C 2^{\epsilon j_{1}} 2^{j_{1}}\left\|\mathcal{F}^{-1}\left(g_{0, j_{1}}\right)\right\|_{L_{x}^{1} L_{t}^{2}} .
\end{gathered}
$$

By examining the supports of the functions, $\eta_{j_{2}}\left(\tau_{2}\right)\left(g_{0, j_{1}} * \mathcal{F}\left(m_{\leq 4, j^{\prime \prime}}\right)\right)\left(\xi_{2}, \tau_{2}\right) \equiv 0$ unless

$$
\left|j_{2}-j_{1}\right| \leq C \text { or } j_{1}, j_{2} \leq j^{\prime \prime}+C
$$

In addition,

$$
\left\|\mathcal{F}^{-1}\left[\eta_{j_{2}}\left(\tau_{2}\right)\left(g_{0, j_{1}} * \mathcal{F}\left(m_{\leq 4, j^{\prime \prime}}\right)\right)\left(\xi_{2}, \tau_{2}\right)\right]\right\|_{L_{x}^{1} L_{t}^{2}} \leq C\left\|\mathcal{F}^{-1}\left(g_{0, j_{1}}\right)\right\|_{L_{x}^{1} L_{t}^{2}} \| m_{\leq 4, j^{\prime \prime}}||_{L_{x, t}^{\infty}} .
$$

The bound (9.15) follows from (9.7) and (9.16).

Assume now that $f_{0}^{\text {high }}=f_{0, j_{1}}^{k^{\prime}}$ is an $L^{2}$ function supported in $D_{k^{\prime}, j_{1}}, k^{\prime} \leq 1$, $\left\|A_{0}^{\epsilon} f_{0}^{\text {high }}\right\|_{Z_{0}} \approx 2^{\epsilon j_{1}} 2^{j_{1}-k^{\prime}}\left\|f_{0, j_{1}}^{k^{\prime}}\right\|_{L^{2}}$. We decompose

$$
m=\sum_{j^{\prime \prime}=0}^{\infty} m_{\leq k^{\prime}-10, j^{\prime \prime}}+\sum_{k^{\prime \prime}=k^{\prime}-9}^{\infty} \sum_{j^{\prime \prime}=0}^{\infty} m_{k^{\prime \prime}, j^{\prime \prime}} .
$$


We observe that $f_{0, j_{1}}^{k^{\prime}} * \mathcal{F}\left(m_{\leq k^{\prime}-10, j^{\prime \prime}}\right)$ is supported in the set $\left\{\left(\xi_{2}, \tau_{2}\right):\left|\xi_{2}\right| \in\right.$ $\left.\left[2^{k^{\prime}-2}, 2^{k^{\prime}+2}\right]\right\}$. In addition, $\eta_{j_{2}}\left(\tau_{2}\right)\left(f_{0, j_{1}}^{k^{\prime}} * \mathcal{F}\left(m_{\leq k^{\prime}-3, j^{\prime \prime}}\right)\right)\left(\xi_{2}, \tau_{2}\right) \equiv 0$ unless (9.16) holds. The same argument as before, using Plancherel's theorem and the bound (9.7), shows that

$$
\left\|\eta_{0}\left(\xi_{2}\right) A_{0}^{\epsilon}\left(\xi_{2}, \tau_{2}\right)\left[f_{0, j_{1}}^{k^{\prime}} * \sum_{j^{\prime \prime}=0}^{\infty} \mathcal{F}\left(m_{\leq k^{\prime}-10, j^{\prime \prime}}\right)\right]\left(\xi_{2}, \tau_{2}\right)\right\|_{X_{0}} \leq C 2^{\epsilon j_{1}} 2^{j_{1}-k^{\prime}}\left\|f_{0, j_{1}}^{k^{\prime}}\right\|_{L^{2}} .
$$

To handle the part corresponding to the second sum in the right-hand side of (9.17), we use the space $Y_{0}$. It suffices to prove that

$$
\begin{array}{r}
\sum_{k^{\prime \prime}=k^{\prime}-9}^{5} \sum_{j^{\prime \prime}=0}^{\infty} \sum_{j_{2}=0}^{\infty} 2^{\epsilon j_{2}} 2^{j_{2}}\left\|\mathcal{F}^{-1}\left[\eta_{j_{2}}\left(\tau_{2}\right)\left(f_{0, j_{1}}^{k^{\prime}} * \mathcal{F}\left(m_{k^{\prime \prime}, j^{\prime \prime}}\right)\right)\left(\xi_{2}, \tau_{2}\right)\right]\right\|_{L_{x}^{1} L_{t}^{2}} \\
\leq C 2^{\epsilon j_{1}} 2^{j_{1}-k^{\prime}}\left\|f_{0, j_{1}}^{k^{\prime}}\right\|_{L^{2}} .
\end{array}
$$

As before, we may assume that $j_{2}$ satisfies the restriction (9.16) and estimate

$$
\begin{aligned}
\left\|\mathcal{F}^{-1}\left[\eta_{j_{2}}\left(\tau_{2}\right)\left(f_{0, j_{1}}^{k^{\prime}} * \mathcal{F}\left(m_{k^{\prime \prime}, j^{\prime \prime}}\right)\right)\left(\xi_{2}, \tau_{2}\right)\right]\right\|_{L_{x}^{1} L_{t}^{2}} & \leq C\left\|\mathcal{F}^{-1}\left(f_{0, j_{1}}^{k^{\prime}}\right)\right\|_{L_{x, t}^{2}}\left\|m_{k^{\prime \prime}, j^{\prime \prime}}\right\|_{L_{x}^{2} L_{t}^{\infty}} \\
& \leq C 2^{-80 j^{\prime \prime}} 2^{-k^{\prime \prime}}\left\|f_{0, j_{1}}^{k^{\prime}}\right\|_{L_{x, t}^{2}},
\end{aligned}
$$

using Plancherel's theorem and (9.7). The bound (9.18) follows.

We prove now the bound (9.5) in the case $k_{2}=0$ and $k_{1} \in[1,10]$. As before, we may assume $f_{k_{1}}^{\text {high }}=f_{k_{1}, j_{1}}$ is an $L^{2}$ function supported in $D_{k_{1}, j_{1}}, j_{1} \geq 0$, $\left\|A_{k_{1}}^{\epsilon} f_{k_{1}}^{\text {high }}\right\|_{Z_{k_{1}}} \approx 2^{\epsilon j_{1}} 2^{j_{1} / 2} \beta_{k_{1}, j_{1}}\left\|f_{k_{1}, j}\right\|_{L^{2}} \approx 2^{\epsilon j_{1}} 2^{j_{1}}\left\|f_{k_{1}, j}\right\|_{L^{2}}$. We use the decomposition (9.17) in the case $k^{\prime}=1$. The proof of the bound (9.5) is then identical to the proof in the case considered before $k_{1}=0, f_{0}^{\text {high }}=f_{0, j_{1}}^{k^{\prime}}, k^{\prime}=1$.

Finally, in the case $k_{1}=0, k_{2} \in[1,10]$, we have the stronger bound

$$
\left\|\eta_{k_{2}}\left(\xi_{2}\right) A_{k_{2}}^{\epsilon}\left(\xi_{2}, \tau_{2}\right) \mathcal{F}\left[m \cdot \mathcal{F}^{-1}\left(f_{0}^{\mathrm{high}}\right)\right]\left(\xi_{2}, \tau_{2}\right)\right\|_{Z_{k_{2}}} \leq C\left\|A_{0}^{\epsilon} f_{0}^{\mathrm{high}}\right\|_{\bar{Z}_{0}},
$$

where $\bar{Z}_{0}$ is defined in (3.8). The proof of this bound is identical to the proof of (9.5) in the case considered before $k_{1}=1, k_{2} \geq 1$.

In some estimates the delicate structure of the spaces $Z_{k}$ is not necessary. For $\alpha \in[-20,20]$ and $k \geq 1$ we define

$$
\begin{aligned}
E_{k, \alpha}= & \left\{f \in L^{2}: f \text { supported in } I_{k} \times \mathbb{R}\right. \text { and } \\
& \left.\|f\|_{E_{k, \alpha}}:=2^{\alpha k} \sum_{j=0}^{\infty} 2^{j}\left\|\eta_{j}(\tau) f(\xi, \tau)\right\|_{L_{\xi, \tau}^{2}}<\infty\right\} .
\end{aligned}
$$

For $k=0$, for simplicity of notation we define $E_{0, \alpha}=Z_{0}$. We notice that

$$
E_{k, 4} \subseteq Z_{k} \subseteq E_{k,-4} \text { for any } k \in \mathbb{Z}_{+} .
$$

Lemma 9.2. (a) Assume $k_{1} \in \mathbb{Z}_{+}, k_{2} \in[1, \infty) \cap \mathbb{Z}$, and $I_{1} \subseteq \widetilde{I}_{k_{1}}, I_{2} \subseteq \widetilde{I}_{k_{2}}$ are intervals. Then, for $m \in S_{100}^{\infty}, \alpha \in[-20,20], \epsilon \in\{-1,0\}$, and $f_{k_{1}} \in E_{k_{1}, \alpha}$

$$
\begin{aligned}
& \| \mathbf{1}_{I_{2}}\left(\xi_{2}\right)\left(\tau_{2}+i\right)^{\epsilon} \cdot \mathcal{F}\left[m \cdot \mathcal{F}^{-1}\left(\mathbf{1}_{I_{1}}\left(\xi_{1}\right) f_{k_{1}}\right)\right] \\
& \quad \leq C\left[1+d\left(I_{1}, I_{2}\right)\right]_{E_{k_{2}, \alpha}} \\
& \quad \leq 0 \mid\|m\|_{S_{100}^{\infty}} \cdot\left\|\left(\tau_{1}+i\right)^{\epsilon} f_{k_{1}}\right\|_{E_{k_{1}, \alpha}},
\end{aligned}
$$

where $d\left(I_{1}, I_{2}\right)$ denotes the distance between the sets $I_{1}$ and $I_{2}$. 
(b) Assume $k_{1} \in \mathbb{Z}_{+}$. Then, for $m \in S_{100}^{\infty}, \alpha \in[-20,20], \epsilon \in\{-1,0\}$, and $f_{k_{1}} \in E_{k_{1}, \alpha}$

$$
\left\|\eta_{0}\left(\xi_{2}\right)\left(\tau_{2}+i\right)^{\epsilon} \cdot \mathcal{F}\left[m \cdot \mathcal{F}^{-1}\left(f_{k_{1}}\right)\right]\right\|_{E_{0, \alpha}} \leq C 2^{-50 k_{1}}\|m\|_{S_{100}^{\infty}} \cdot\left\|\left(\tau_{1}+i\right)^{\epsilon} f_{k_{1}}\right\|_{E_{k_{1}, \alpha}} .
$$

Proof of Lemma 9.2. We may assume $\|m\|_{S_{100}^{\infty}}=1$ and argue as in the proof of Lemma 9.1. We may assume $f_{k_{1}}=f_{k_{1}, j_{1}}$ is an $L^{2}$ function supported in $\widetilde{I}_{k_{1}} \times \widetilde{I}_{j_{1}}$, $\left\|\left(\tau_{1}+i\right)^{\epsilon} f_{k_{1}}\right\|_{E_{k_{1}, \alpha}} \geq C^{-1} 2^{\alpha k_{1}} 2^{\epsilon j_{1}} 2^{j_{1}}|| f_{k_{1}, j_{1}} \|_{L^{2}}$. With the notation in Lemma 9.1 . we write

$$
m=\sum_{j^{\prime \prime}=0}^{\infty} m_{\leq 0, j^{\prime \prime}}+\sum_{k^{\prime \prime}=1}^{\infty} \sum_{j^{\prime \prime}=0}^{\infty} m_{k^{\prime \prime}, j^{\prime \prime}} .
$$

For (9.22) it suffices to prove that

$$
\begin{aligned}
& 2^{\alpha k_{2}} \sum_{j_{2}, j^{\prime \prime} \geq 0} 2^{\epsilon j_{2}} 2^{j_{2}}\left\|\eta_{j_{2}}\left(\tau_{2}\right) \mathbf{1}_{I_{2}}\left(\xi_{2}\right) \cdot\left[\left(\mathbf{1}_{I_{1}}\left(\xi_{1}\right) f_{k_{1}, j_{1}}\right) * \mathcal{F}\left(m_{\leq 0, j^{\prime \prime}}\right)\right]\right\|_{L^{2}} \\
& +2^{\alpha k_{2}} \sum_{k^{\prime \prime} \geq 1} \sum_{j_{2}, j^{\prime \prime} \geq 0} 2^{\epsilon j_{2}} 2^{j_{2}}\left\|\eta_{j_{2}}\left(\tau_{2}\right) \mathbf{1}_{I_{2}}\left(\xi_{2}\right) \cdot\left[\left(\mathbf{1}_{I_{1}}\left(\xi_{1}\right) f_{k_{1}, j_{1}}\right) * \mathcal{F}\left(m_{k^{\prime \prime}, j^{\prime \prime}}\right)\right]\right\|_{L^{2}} \\
& \leq C\left[1+d\left(I_{1}, I_{2}\right)\right]^{-50} 2^{\alpha k_{1}} 2^{\epsilon j_{1}} 2^{j_{1}}\left\|f_{k_{1}, j_{1}}\right\|_{L^{2}} .
\end{aligned}
$$

By examining the supports of the functions, we see that the first sum in the left-hand side of (9.25) is nontrivial only if $d\left(I_{1}, I_{2}\right) \leq C$ (so $\left|k_{1}-k_{2}\right| \leq C$ ). In addition, $\eta_{j_{2}}\left(\tau_{2}\right) \mathbf{1}_{I_{2}}\left(\xi_{2}\right) \cdot\left[\left(\mathbf{1}_{I_{1}}\left(\xi_{1}\right) f_{k_{1}, j_{1}}\right) * \mathcal{F}\left(m_{\leq 0, j^{\prime \prime}}\right)\right] \equiv 0$ unless

$$
\left|j_{1}-j_{2}\right| \leq C \text { or } j_{1}, j_{2} \leq j^{\prime \prime}+C \text {. }
$$

Using Plancherel's theorem and (9.7),

$$
\left\|\left(\mathbf{1}_{I_{1}}\left(\xi_{1}\right) f_{k_{1}, j_{1}}\right) * \mathcal{F}\left(m_{\leq 0, j^{\prime \prime}}\right)\right\|_{L^{2}} \leq C 2^{-80 j^{\prime \prime}}\left\|f_{k_{1}, j_{1}}\right\|_{L^{2}} .
$$

The bound (9.25) for the first sum follows easily. For the second sum, we may assume that $2^{k^{\prime \prime}} \geq C^{-1} d\left(I_{1}, I_{2}\right)$ (so $2^{k^{\prime \prime}} \geq C^{-1} 2^{\left|k_{1}-k_{2}\right|}$ ) and that the restriction (9.26) still holds. Using Plancherel's theorem and (9.7),

$$
\left\|\left(\mathbf{1}_{I_{1}}\left(\xi_{1}\right) f_{k_{1}, j_{1}}\right) * \mathcal{F}\left(m_{k^{\prime \prime}, j^{\prime \prime}}\right)\right\|_{L^{2}} \leq C 2^{-80 k^{\prime \prime}} 2^{-80 j^{\prime \prime}}\left\|f_{k_{1}, j_{1}}\right\|_{L^{2}} .
$$

The bound (9.25) for the second sum follows easily. This completes the proof of part (a).

For part (b), we may assume $k_{1} \geq 10$ (in view of Lemma 9.2) and $f_{k_{1}}=f_{k_{1}, j_{1}}$ is as before. We decompose $m$ as in (9.24). For (9.23) it suffices to prove that

$$
\begin{gathered}
\sum_{\left|k^{\prime \prime}-k_{1}\right| \leq 2} \sum_{j_{2}, j^{\prime \prime} \geq 0} 2^{\epsilon j_{2}} 2^{j_{2}}\left\|\mathcal{F}^{-1}\left[\eta_{j_{2}}\left(\tau_{2}\right) \eta_{0}\left(\xi_{2}\right) \cdot\left(f_{k_{1}, j_{1}} * \mathcal{F}\left(m_{k^{\prime \prime}, j^{\prime \prime}}\right)\right)\right]\right\|_{L_{x}^{1} L_{t}^{2}} \\
\leq C 2^{-50 k_{1}} 2^{\alpha k_{1}} 2^{\epsilon j_{1}} 2^{j_{1}}\left\|f_{k_{1}, j_{1}}\right\|_{L^{2}} .
\end{gathered}
$$

We may also assume that the restriction (9.26) holds. Using Plancherel's theorem and (9.7),

$$
\begin{aligned}
& \left\|\mathcal{F}^{-1}\left[\eta_{j_{2}}\left(\tau_{2}\right) \eta_{0}\left(\xi_{2}\right) \cdot\left(f_{k_{1}, j_{1}} * \mathcal{F}\left(m_{k^{\prime \prime}, j^{\prime \prime}}\right)\right)\right]\right\|_{L_{x}^{1} L_{t}^{2}} \\
& \quad \leq C\left\|m_{k^{\prime \prime}, j^{\prime \prime}}\right\|\left\|_{L_{x}^{2} L_{t}^{\infty}}\right\| \mathcal{F}^{-1}\left(f_{k_{1}, j_{1}}\right) \|_{L^{2}} \\
& \quad \leq C 2^{-80 k^{\prime \prime}} 2^{-80 j^{\prime \prime}}\left\|f_{k_{1}, j_{1}}\right\|_{L^{2}}
\end{aligned}
$$


The bound (9.27) follows easily. This completes the proof of part (b).

We state now a slightly stronger form of Lemma 9.1 that will be used in the next section.

Corollary 9.3. (a) If $k_{1}, k_{2} \in \mathbb{Z}_{+}, \epsilon \in\{-1,0\}, f_{k_{1}}^{\text {high }} \in Z_{k_{1}}^{\text {high }}$, and $m \in S_{100}^{\infty}$, then (9.28)

$\left\|\eta_{k_{2}}\left(\xi_{2}\right) A_{k_{2}}^{\epsilon}\left(\xi_{2}, \tau_{2}\right) \cdot \mathcal{F}\left[m \cdot \mathcal{F}^{-1}\left(f_{k_{1}}^{\mathrm{high}}\right)\right]\right\|_{Z_{k_{2}}} \leq C 2^{-30\left|k_{1}-k_{2}\right|}\|m\|_{S_{100}^{\infty}}\left\|A_{k_{1}}^{\epsilon} f_{k_{1}}^{\mathrm{high}}\right\|_{Z_{k_{1}}}$.

(b) If $k_{2} \in \mathbb{Z}_{+}, \epsilon \in\{-1,0\}, f_{0} \in \bar{Z}_{0}$, and $m^{\prime} \in S_{100}^{2}$, then

$$
\left\|\eta_{k_{2}}\left(\xi_{2}\right) A_{k_{2}}^{\epsilon}\left(\xi_{2}, \tau_{2}\right) \cdot \mathcal{F}\left[m^{\prime} \cdot \mathcal{F}^{-1}\left(f_{0}\right)\right]\right\|_{Z_{k_{2}}} \leq C 2^{-30 k_{2}}\left\|m^{\prime}\right\|_{S_{100}^{2}}\left\|A_{0}^{\epsilon} f_{0}\right\|_{\bar{Z}_{0}} .
$$

Proof of Corollary 9.3, Part (a) follows from Lemma 9.1, Lemma 9.2, and (9.21). For part (b), we notice that $\left\|m_{\leq k^{\prime \prime}, j^{\prime \prime}}^{\prime}\right\|_{L_{x}^{2} L_{t}^{\infty}} \leq C 2^{-80 j^{\prime \prime}}$ for any $k^{\prime \prime} \in \mathbb{Z}, j^{\prime \prime} \in \mathbb{Z}_{+}$. The bound (9.29) then follows from the proof of (9.15), the bound (9.19), and the proof of Lemma 9.2(a) with $k_{1}=1$.

\section{Proof of Theorem 1.1}

In this section we complete the proof of Theorem 1.1. The main ingredients are Lemma 2.1, Lemma 5.1] Lemma [5.2, Proposition 7.1] Proposition 7.2, Proposition 8.1. Proposition 8.2. Lemma 9.2, and Corollary 9.3. We start by showing that the data $e^{ \pm i U_{0}(., 0)} P_{ \pm \text {high }} \phi$ of the initial-value problems (2.10) and (2.12) are in $\widetilde{H}^{\sigma}$, $\sigma \geq 0$.

Lemma 10.1. Assume $U: \mathbb{R} \rightarrow \mathbb{R}$ satisfies the bounds

$$
\left\|\partial_{x}^{\sigma_{2}} U\right\|_{L_{x}^{2}} \leq 1 \text { for any } \sigma_{2} \in[1,110] \cap \mathbb{Z} .
$$

Then, for any $\sigma \in[0,20]$ and $\phi \in H^{\sigma}$,

$$
\left\|e^{ \pm i U} P_{ \pm \text {high }} \phi\right\|_{\widetilde{H}^{\sigma}} \leq C\|\phi\|_{H^{\sigma}}
$$

Proof of Lemma 10.1. To fix the notation, assume that the sign in the left-hand side of (10.2) is + . So we may assume that $\widehat{\phi}$ is supported in the interval $\left[2^{10}, \infty\right)$. For any $k^{\prime \prime} \in \mathbb{Z}$ let

$$
V_{k^{\prime \prime}}=\mathcal{F}_{1}^{-1}\left[\chi_{k^{\prime \prime}}(\xi) \mathcal{F}_{1}\left[e^{i U(x)}\right]\right]
$$

and $V_{\leq k^{\prime \prime}}=\sum_{k^{\prime \prime \prime} \leq k^{\prime \prime}} V_{k^{\prime \prime \prime}}$. Using (10.1) and the Sobolev imbedding theorem,

$$
\left\|V_{\leq 0}\right\|_{L_{x}^{\infty}} \leq C \text { and }\left\|V_{k^{\prime \prime}}\right\|_{L_{x}^{2}}+\left\|V_{k^{\prime \prime}}\right\|_{L_{x}^{\infty}} \leq C 2^{-80 k^{\prime \prime}} \text { for any } k^{\prime \prime} \geq 1
$$

We turn now to the proof of (10.2). For any $k_{1} \geq 10$ let $\phi_{k_{1}}=P_{k_{1}} \phi$. In view of the definitions, it suffices to prove that

$$
\left\{\begin{array}{l}
\left\|P_{k_{2}}\left(e^{i U} \phi_{k_{1}}\right)\right\|_{L^{2}} \leq C 2^{-40\left|k_{1}-k_{2}\right|}\left\|\phi_{k_{1}}\right\|_{L^{2}} \text { if } k_{2} \geq 1 ; \\
\left\|P_{0}\left(e^{i U} \phi_{k_{1}}\right)\right\|_{L^{1}} \leq C 2^{-40 k_{1}}\left\|\phi_{k_{1}}\right\|_{L^{2}} .
\end{array}\right.
$$

For the first bound in (10.5), if $\left|k_{1}-k_{2}\right| \leq 10$, then $\left\|P_{k_{2}}\left(e^{i U} \phi_{k_{1}}\right)\right\|_{L^{2}} \leq C\left\|\phi_{k_{1}}\right\|_{L^{2}}$ as desired. If $\left|k_{1}-k_{2}\right| \geq 10$, then

$$
\begin{aligned}
\left\|P_{k_{2}}\left(e^{i U} \phi_{k_{1}}\right)\right\|_{L^{2}} & \leq \sum_{k^{\prime \prime} \geq\left|k_{1}-k_{2}\right|-C}\left\|P_{k_{2}}\left(V_{k^{\prime \prime}} \phi_{k_{1}}\right)\right\|_{L^{2}} \\
& \leq C \sum_{k^{\prime \prime} \geq\left|k_{1}-k_{2}\right|-C}\left\|V_{k^{\prime \prime}}\right\|_{L^{\infty}}\left\|\phi_{k_{1}}\right\|_{L^{2}},
\end{aligned}
$$


which suffices in view of (10.4). For the second bound in (10.5), since $k_{1} \geq 10$,

$$
\left\|P_{0}\left(e^{i U} \phi_{k_{1}}\right)\right\|_{L^{1}} \leq \sum_{\left|k^{\prime \prime}-k_{1}\right| \leq 2}\left\|P_{0}\left(V_{k^{\prime \prime}} \phi_{k_{1}}\right)\right\|_{L^{1}} \leq C \sum_{\left|k^{\prime \prime}-k_{1}\right| \leq 2}\left\|V_{k^{\prime \prime}}\right\|_{L^{2}}\left\|\phi_{k_{1}}\right\|_{L^{2}}
$$

which suffices in view of (10.4).

We prove now our main bilinear estimate for functions in $F^{\sigma}$.

Proposition 10.2. If $m \in S_{110}^{\infty}, m^{\prime} \in S_{110}^{2}, \sigma \in[0,20]$, and $u, v \in F^{\sigma}$, then

$$
\begin{aligned}
& \left\|\partial_{x}(m \cdot u v)\right\|_{N^{\sigma}}+\left\|m^{\prime} \cdot(u v)\right\|_{N^{\sigma}} \\
& \quad \leq C\left(\|m\|_{S_{110}^{\infty}}+\left\|m^{\prime}\right\|_{S_{110}^{2}}\right)\left(\|u\|_{F^{\sigma}}\|v\|_{F^{0}}+\|u\|_{F^{0}}\|v\|_{F^{\sigma}}\right) .
\end{aligned}
$$

Proof of Proposition 10.2. We show first that

$$
\left\|\partial_{x}(u v)\right\|_{N^{\sigma}} \leq C\left(\|u\|_{F^{\sigma}}\|v\|_{F^{0}}+\|u\|_{F^{0}}\|v\|_{F^{\sigma}}\right) .
$$

For $k \in \mathbb{Z}_{+}$let $F_{k}(\xi, \tau)=\eta_{k}(\xi) \mathcal{F}(u)(\xi, \tau)$ and $G_{k}(\xi, \tau)=\eta_{k}(\xi) \mathcal{F}(v)(\xi, \tau)$. Then

$$
\left\{\begin{array}{l}
\|u\|_{F^{\sigma}}^{2}=\sum_{k_{1}=0}^{\infty} 2^{2 \sigma k_{1}}\left\|\left(I-\partial_{\tau}^{2}\right) F_{k_{1}}\right\|_{Z_{k_{1}}}^{2} \\
\|v\|_{F^{\sigma}}^{2}=\sum_{k_{2}=0}^{\infty} 2^{2 \sigma k_{2}}\left\|\left(I-\partial_{\tau}^{2}\right) G_{k_{2}}\right\|_{Z_{k_{2}}}^{2}
\end{array}\right.
$$

and

$$
\eta_{k}(\xi) \mathcal{F}\left[\partial_{x}(u \cdot v)\right](\xi, \tau)=C \xi \sum_{k_{1}, k_{2} \in \mathbb{Z}} \eta_{k}(\xi)\left[F_{k_{1}} * G_{k_{2}}\right](\xi, \tau)
$$

We observe that $\eta_{k}(\xi)\left[F_{k_{1}} * G_{k_{2}}\right](\xi, \tau) \equiv 0$ unless

$$
\left\{\begin{array}{l}
k_{1} \leq k-10 \text { and } k_{2} \in[k-2, k+2] \text { or } \\
k_{1} \in[k-2, k+2] \text { and } k_{1} \leq k-10 \text { or } \\
k_{1}, k_{2} \in[k-10, k+20] \text { or } \\
k_{1}, k_{2} \geq k+10 \text { and }\left|k_{1}-k_{2}\right| \leq 2
\end{array}\right.
$$

For $k, k_{1}, k_{2} \in \mathbb{Z}$ let

$$
H_{k, k_{1}, k_{2}}(\xi, \tau)=\eta_{k}(\xi) A_{k}(\xi, \tau)^{-1} \xi \cdot\left(F_{k_{1}} * G_{k_{2}}\right)(\xi, \tau) .
$$

Using the definitions,

$$
\left\|\partial_{x}(u \cdot v)\right\|_{N^{\sigma}}^{2}=C \sum_{k \geq 0} 2^{2 \sigma k}\left\|\sum_{k_{1}, k_{2}} H_{k, k_{1}, k_{2}}\right\|_{Z_{k}}^{2}
$$


For $k \in \mathbb{Z}_{+}$fixed we estimate, using Propositions 7.1, 7.2, 8.1, and 8.2 ,

$$
\begin{aligned}
& \left\|\sum_{k_{1}, k_{2}} H_{k, k_{1}, k_{2}}\right\|_{Z_{k}} \\
& \leq \sum_{\left|k_{2}-k\right| \leq 2}\left\|\sum_{k_{1} \leq k-10} H_{k, k_{1}, k_{2}}\right\|_{Z_{k}}+\sum_{\left|k_{1}-k\right| \leq 2}\left\|\sum_{k_{2} \leq k-10} H_{k, k_{1}, k_{2}}\right\|_{Z_{k}} \\
& +\sum_{k_{1}, k_{2} \in[k-10, k+20]}\left\|H_{k, k_{1}, k_{2}}\right\|_{Z_{k}}+\sum_{k_{1}, k_{2} \geq k+10,\left|k_{1}-k_{2}\right| \leq 2}\left\|H_{k, k_{1}, k_{2}}\right\|_{Z_{k}} \\
& \leq C\left[\sum_{\left|k_{2}-k\right| \leq 2}\left\|G_{k_{2}}\right\|_{Z_{k_{2}}}\right] \cdot\|u\|_{F^{0}}+C\left[\sum_{\left|k_{1}-k\right| \leq 2}\left\|F_{k_{1}}\right\|_{Z_{k_{1}}}\right] \cdot\|v\|_{F^{0}} \\
& +C\left[\sum_{\left|k_{1}-k\right| \leq 20}\left\|F_{k_{1}}\right\|_{Z_{k_{1}}}\right]\left[\sum_{\left|k_{2}-k\right| \leq 20}\left\|G_{k_{2}}\right\|_{Z_{k_{2}}}\right] \\
& +C 2^{-k / 4}\left[\sum_{k_{1} \geq k}\left\|F_{k_{1}}\right\|_{Z_{k_{1}}}^{2}\right]^{1 / 2}\left[\sum_{k_{2} \geq k}\left\|G_{k_{2}}\right\|_{Z_{k_{2}}}^{2}\right]^{1 / 2} .
\end{aligned}
$$

The bound (10.7) follows. A similar estimate, using Proposition 8.1 and (8.17), shows that

$$
\begin{aligned}
\left\|\eta_{0}(\xi) A_{0}(\xi, \tau)^{-1} \mathcal{F}(u v)\right\|_{\bar{Z}_{0}} & +\left[\sum_{k \geq 1} 2^{2 \sigma k}\left\|\eta_{k}(\xi) A_{k}(\xi, \tau)^{-1} \mathcal{F}(u v)\right\|_{Z_{k}}^{2}\right]^{1 / 2} \\
& \leq C\left(\|u\|_{F^{\sigma}}\|v\|_{F^{0}}+\|u\|_{F^{0}}\|v\|_{F^{\sigma}}\right) .
\end{aligned}
$$

We would like now to use the bound (9.28) to include the factor $m$. We may assume $\|m\|_{S_{110}^{\infty}}=1$. For any $u \in C\left(\mathbb{R}: H^{-2}\right)$ we write $u=u^{\text {low }}+u^{\text {high }}$,

$$
\left\{\begin{array}{l}
u^{\text {low }}=\sum_{k \geq 0} \mathcal{F}^{-1}\left[\eta_{k}(\xi) \mathcal{F}(u)(\xi, \tau) \cdot \eta_{\leq k-15}(\tau-\omega(\xi))\right]=\sum_{k \geq 0} \mathcal{F}^{-1}\left(f_{k}^{\text {low }}\right) ; \\
u^{\text {high }}=\sum_{k \geq 0} \mathcal{F}^{-1}\left[\eta_{k}(\xi) \mathcal{F}(u)(\xi, \tau) \cdot\left(1-\eta_{\leq k-15}(\tau-\omega(\xi))\right)\right]=\sum_{k \geq 0} \mathcal{F}^{-1}\left(f_{k}^{\text {high }}\right) .
\end{array}\right.
$$

Then, using (9.28) with $\epsilon=0$,

$$
\begin{aligned}
\left\|m \cdot u^{\mathrm{high}}\right\|_{F^{\sigma}}^{2} & =\sum_{k \geq 0} 2^{2 \sigma k}\left\|\eta_{k}(\xi) \mathcal{F}\left[\left(t^{2}+1\right) m u^{\mathrm{high}}\right]\right\|_{Z_{k}}^{2} \\
& \leq C \sum_{k \geq 0} 2^{2 \sigma k}\left[\sum_{k^{\prime} \geq 0}\left\|\eta_{k}(\xi) \mathcal{F}\left[\left(t^{2}+1\right) m \cdot \mathcal{F}^{-1}\left(f_{k^{\prime}}^{\mathrm{high}}\right)\right]\right\|_{Z_{k}}\right]^{2} \\
& \leq C \sum_{k \geq 0} 2^{2 \sigma k}\left[\sum_{k^{\prime} \geq 0} 2^{-30\left|k-k^{\prime}\right|}\left\|f_{k^{\prime}}^{\mathrm{high}}\right\|_{Z_{k^{\prime}}}\right]^{2} \\
& \leq C\|u\|_{F^{\sigma}}^{2}
\end{aligned}
$$

for any $u \in F^{\sigma}$. A similar estimate, using (9.28) with $\epsilon=-1$, gives

$$
\left\|m \cdot w^{\text {high }}\right\|_{N^{\sigma}} \leq C\|w\|_{N^{\sigma}}
$$

for any $w \in N^{\sigma}$. We estimate now the first term in the left-hand side of (10.6) by

$$
\begin{gathered}
\left\|\partial_{x}\left[\left(m u^{\text {high }}\right) v\right]\right\|\left\|_{N^{\sigma}}+\right\| \partial_{x}\left[u^{\text {low }}\left(m v^{\text {high }}\right)\right] \|_{N^{\sigma}} \\
+\left\|m \cdot \partial_{x}\left(u^{\text {low }} v^{\text {low }}\right)\right\|_{N^{\sigma}}+\left\|\partial_{x} m \cdot\left(u^{\text {low }} v^{\text {low }}\right)\right\|_{N^{\sigma}} .
\end{gathered}
$$

In view of (10.7) and (10.10), the first two terms in (10.12) can be estimated by $C\left(\left.\|u\|\right|_{F^{\sigma}}\|v\|_{F^{0}}+\|u\|_{F^{0}}\|v\|_{F^{\sigma}}\right)$, as desired. For the third term, we use the important 
observation that the product of two low-modulation functions has high modulation:

$$
\left(u^{\text {low }} v^{\text {low }}\right)^{\text {low }} \equiv 0 .
$$

Assuming (10.13), the third term in (10.12) can be estimated by

$$
C\left(\|u\|_{F^{\sigma}}\|v\|_{F^{0}}+\|u\|_{F^{0}}\|v\|_{F^{\sigma}}\right),
$$

using (10.7) and (10.11). To prove (10.13), we write

$$
u^{\text {low }}=\sum_{k \geq 15} \mathcal{F}^{-1}\left(f_{k}^{\text {low }}\right) \text { and } v^{\text {low }}=\sum_{k \geq 15} \mathcal{F}^{-1}\left(g_{k}^{\text {low }}\right),
$$

where $f_{k}^{\text {low }}$ and $g_{k}^{\text {low }}$ are supported in $\bigcup_{j \leq k-15} D_{k, j}$. For (10.13) it suffices to prove that

$$
\eta_{k}(\xi) \eta_{\leq k-15}(\tau-\omega(\xi))\left(f_{k_{1}}^{\text {low }} * g_{k_{2}}^{\text {low }}\right) \equiv 0, \text { where } k, k_{1}, k_{2} \geq 15,
$$

which follows easily from (6.22) and (6.23).

In view of (9.3), for (10.6), it suffices to prove that if $\left\|m^{\prime}\right\|_{S_{100}^{2}}=1$, then

$$
\left\|m^{\prime} \cdot(u v)\right\|_{N^{\sigma}} \leq C\left(\|u\|_{F^{\sigma}}\|v\|_{F^{0}}+\|u\|_{F^{0}}\|v\|_{F^{\sigma}}\right) .
$$

We write $u=u^{\text {high }}+u^{\text {low }}, v=v^{\text {high }}+v^{\text {low }}$ as before. Then, using (10.9), the bound (9.29) with $k_{2}=0$, and Lemma $9.2(\mathrm{~b})$,

$$
\left\|P_{0}\left(m^{\prime} \cdot u v\right)\right\|_{N^{\sigma}} \leq C\left(\|u\|_{F^{\sigma}}\|v\|_{F^{0}}+\|u\|_{F^{0}}\|v\|_{F^{\sigma}}\right) .
$$

Also, using (10.9) and (10.10) as before,

$$
\begin{aligned}
\left\|\left(I-P_{0}\right)\left(m^{\prime} \cdot u^{\text {high }} v\right)\right\|_{N^{\sigma}}+\| & \left(I-P_{0}\right)\left(m^{\prime} \cdot u^{\text {low }} v^{\text {high }}\right) \|_{N^{\sigma}} \\
& \leq C\left(\|u\|_{F^{\sigma}}\|v\|_{F^{0}}+\|u\|_{F^{0}}\|v\|_{F^{\sigma}}\right) .
\end{aligned}
$$

Finally, using (10.11), (9.29), and the observation (10.13),

$$
\left\|\left(I-P_{0}\right)\left(m^{\prime} \cdot u^{\text {low }} v^{\text {low }}\right)\right\|_{N^{\sigma}} \leq C\left(\|u\|_{F^{\sigma}}\|v\|_{F^{0}}+\|u\|_{F^{0}}\|v\|_{F^{\sigma}}\right),
$$

which completes the proof of (10.14).

To bound the error terms in the formulas (2.11) and (2.13) of $E_{+}$and $E_{-}$, we use the less demanding spaces $E_{k, \alpha}$ defined in (9.20) for $k \geq 1$,

$$
\begin{aligned}
E_{k, \alpha}= & \left\{f \in L^{2}: f \text { supported in } I_{k} \times \mathbb{R}\right. \text { and } \\
& \left.\|f\|_{E_{k, \alpha}}:=2^{\alpha k} \sum_{j=0}^{\infty} 2^{j}\left\|\eta_{j}(\tau) f(\xi, \tau)\right\|_{L_{\xi, \tau}^{2}}<\infty\right\} .
\end{aligned}
$$

For $\sigma \geq 0$ and $\alpha \in[-20,20]$ we define

$$
F_{\alpha}^{\sigma}=\left\{u \in \mathcal{S}^{\prime}(\mathbb{R} \times \mathbb{R}):\|u\|_{F_{\alpha}^{\sigma}}^{2}:=\sum_{k=0}^{\infty} 2^{2 \sigma k}\left\|\eta_{k}(\xi)\left(I-\partial_{\tau}^{2}\right) \mathcal{F}(u)\right\|_{E_{k, \alpha}}^{2}<\infty\right\}
$$

and

$$
N_{\alpha}^{\sigma}=\left\{u \in \mathcal{S}^{\prime}(\mathbb{R} \times \mathbb{R}):\|u\|_{N_{\alpha}^{\sigma}}^{2}:=\sum_{k=0}^{\infty} 2^{2 \sigma k}\left\|\eta_{k}(\xi)(\tau+i)^{-1} \mathcal{F}(u)\right\|_{E_{k, \alpha}}^{2}<\infty\right\} .
$$

In view of (9.21),

$$
F_{6}^{\sigma} \subseteq F^{\sigma} \subseteq F_{-6}^{\sigma} \text { and } N_{6}^{\sigma} \subseteq N^{\sigma} \subseteq N_{-6}^{\sigma}
$$


Lemma 10.3. If $m \in S_{110}^{\infty}, \sigma \in[0,20], \alpha \in[-20,20]$, and $u \in F_{\alpha}^{\sigma}$, then

$$
\left\{\begin{array}{l}
\|m \cdot u\|_{F_{\alpha}^{\sigma}} \leq C\|m\|_{S_{110}^{\infty}}\|u\|_{F_{\alpha}^{\sigma}} ; \\
\|m \cdot u\|_{N_{\alpha}^{\sigma}} \leq C\|m\|_{S_{110}^{\infty}}\|u\|_{N_{\alpha}^{\sigma}} .
\end{array}\right.
$$

Proof of Lemma 10.3. We may assume $\|m\|_{S_{110}^{\infty}}=1$. Let $f_{k^{\prime}}=\eta_{k^{\prime}}(\xi) \mathcal{F}(u), k^{\prime} \in$ $\mathbb{Z}_{+}$. Using Lemma 9.2 with $\epsilon=0$, we have

$$
\begin{aligned}
\|m \cdot u\|_{F_{\alpha}^{\sigma}}^{2} & =\sum_{k \geq 0} 2^{2 \sigma k}\left\|\eta_{k}(\xi) \mathcal{F}\left[\left(t^{2}+1\right) m \cdot u\right]\right\|_{E_{k, \alpha}}^{2} \\
& \leq C \sum_{k \geq 0} 2^{2 \sigma k}\left[\sum_{k^{\prime} \geq 0}\left\|\eta_{k}(\xi) \mathcal{F}\left[\left(t^{2}+1\right) m \cdot \mathcal{F}^{-1}\left(f_{k^{\prime}}\right)\right]\right\|_{E_{k, \alpha}}\right]^{2} \\
& \leq C \sum_{k \geq 0} 2^{2 \sigma k}\left[\sum_{k^{\prime} \geq 0} 2^{-50\left|k-k^{\prime}\right|}\left\|f_{k^{\prime}}\right\|_{E_{k^{\prime}, \alpha}}\right]^{2} \\
& \leq C\|u\|_{F_{\alpha}^{\sigma}}^{2} .
\end{aligned}
$$

A similar estimate using Lemma 9.2 with $\epsilon=-1$ gives the second bound in (10.16).

Lemma 10.4. (a) Assume that $I \neq I^{\prime} \in\left\{\left[-2^{10}, 2^{10}\right],\left[2^{10}, \infty\right),\left(-\infty,-2^{10}\right]\right\}$ and $m \in S_{110}^{\infty}$. Then, for any $\sigma \in[0,20]$ and $u \in F_{-10}^{\sigma}$,

$$
\left\{\begin{array}{l}
\left\|P_{I}\left[m \cdot P_{I^{\prime}}(u)\right]\right\|_{F_{10}^{\sigma}} \leq C\|m\|_{S_{110}^{\infty}}\|u\|_{F_{-10}^{\sigma}} ; \\
\left\|P_{I}\left[m \cdot P_{I^{\prime}}(u)\right]\right\|_{N_{10}^{\sigma}} \leq C\|m\|_{S_{110}^{\infty}}\|u\|_{N_{-10}^{\sigma}},
\end{array}\right.
$$

where $P_{I}$ denotes the operator defined by the multiplier $(\xi, \tau) \rightarrow \mathbf{1}_{I}(\xi)$.

(b) In addition, for any $\sigma \in[0,20]$ and $u \in F_{-10}^{\sigma}$,

$$
\left\|\partial_{x}^{2} P_{-}\left(m \cdot P_{+ \text {high }}(u)\right)\right\|_{F_{10}^{\sigma}}+\left\|\partial_{x}^{2} P_{+}\left(m \cdot P_{- \text {high }}(u)\right)\right\|_{F_{10}^{\sigma}} \leq C\|m\|_{S_{110}^{\infty}}\|u\|_{F_{-10}^{\sigma}} .
$$

Proof of Lemma 10.4. We may assume $\|m\|_{S_{110}^{\infty}}=1$ and use Lemma 9.2 and the definitions. The main observation is that if $k, k^{\prime} \in \mathbb{Z}_{+}$, then $d\left(I \cap \widetilde{I}_{k}, I^{\prime} \cap \widetilde{I}_{k^{\prime}}\right) \geq$ $C^{-1}\left(2^{k}+2^{k^{\prime}}\right)$. Let $f_{k^{\prime}}=\eta_{k^{\prime}}(\xi) \cdot \mathcal{F}(u)(\xi, \tau)$. Using Lemma 9.2 with $\epsilon=0$, we have

$$
\begin{aligned}
\| P_{I}\left[m \cdot P_{I^{\prime}}\right. & (u)]\left\|_{F_{10}^{\sigma}}^{2}=\sum_{k \geq 0} 2^{2 \sigma k}\right\| \eta_{k}(\xi) \mathbf{1}_{I}(\xi) \cdot \mathcal{F}\left[\left(t^{2}+1\right) m \cdot P_{I^{\prime}}(u)\right] \|_{E_{k, 10}}^{2} \\
& \leq C \sum_{k \geq 0} 2^{2 \sigma k}\left[\sum_{k^{\prime} \geq 0}\left\|\eta_{k}(\xi) \mathbf{1}_{I}(\xi) \cdot \mathcal{F}\left[\left(t^{2}+1\right) m \cdot \mathcal{F}^{-1}\left(\mathbf{1}_{I^{\prime}} f_{k^{\prime}}\right)\right]\right\|_{E_{k, 10}}\right]^{2} \\
& \leq C \sum_{k \geq 0} 2^{2 \sigma k}\left[\sum_{k^{\prime} \geq 0}\left(2^{k}+2^{k^{\prime}}\right)^{-50} 2^{20 k^{\prime}} 2^{-\sigma k^{\prime}} 2^{\sigma k^{\prime}}\left\|f_{k^{\prime}}\right\|_{E_{k^{\prime},-10}}\right]^{2} \\
& \leq C\|u\|_{F_{-10}^{\sigma}}^{2} .
\end{aligned}
$$

A similar estimate using Lemma 9.2 with $\epsilon=-1$ gives the second bound in (10.17).

For part (b) the same argument as before works, except for the dyadic piece corresponding to $k=0$ (in the left-hand side). To handle this dyadic piece, we need the additional observation

$$
\left\|\xi^{2} \mathbf{1}_{ \pm}(\xi) \eta_{0}(\xi) f\right\|_{Z_{0}} \leq\left\|\xi^{2} \eta_{0}(\xi) f\right\|_{X_{0}} \leq C\left\|\eta_{0}(\xi) f\right\|_{\bar{Z}_{0}} \leq C\left\|\eta_{0}(\xi) f\right\|_{Z_{0}}
$$

where $\mathbf{1}_{ \pm}$denotes the characteristic function of the interval $\{\xi: \pm \xi \in[0, \infty)\}$. 
We can now analyze the nonlinear terms $E_{+}, E_{-}$, and $E_{0}$ in (2.11), (2.13), and (2.15). We assume that $u_{0}, U_{0}: \mathbb{R} \times[-2,2] \rightarrow \mathbb{R}$ are fixed functions that satisfy the bounds (compare with (2.1) and (2.9))

$$
\left\{\begin{array}{l}
\left\|\partial_{t}^{\sigma_{1}} \partial_{x}^{\sigma_{2}} u_{0}\right\|_{L_{x, t}^{2}} \leq \delta \text { for any } \sigma_{1}, \sigma_{2} \in[0,120] \cap \mathbb{Z} \\
\left\|\partial_{t}^{\sigma_{1}} \partial_{x}^{\sigma_{2}} U_{0}\right\|_{L_{x, t}^{2}} \leq \delta \text { for any } \sigma_{1} \in[0,120] \cap \mathbb{Z}, \sigma_{2} \in[1,120] \cap \mathbb{Z}
\end{array}\right.
$$

for some $\delta \ll 1$, and $E_{+}, E_{-}$, and $E_{0}$ are defined as in (2.11), (2.13), and (2.15). For simplicity of notation, let $\mathbf{w}=\left(w_{+}, w_{-}, w_{0}\right)$ and

$$
\mathbf{E}(\mathbf{w})=\left(E_{+}\left(w_{+}, w_{-}, w_{0}\right), E_{-}\left(w_{+}, w_{-}, w_{0}\right), E_{0}\left(w_{+}, w_{-}, w_{0}\right)\right) .
$$

For any Banach space $B$ let $\|\mathbf{w}\|_{B}=\left\|w_{+}\right\|_{B}+\left\|w_{-}\right\|_{B}+\left\|w_{0}\right\|_{B}$ and

$$
\|\mathbf{E}(\mathbf{w})\|_{B}=\left\|E_{+}\left(w_{+}, w_{-}, w_{0}\right)\right\|_{B}+\left\|E_{-}\left(w_{+}, w_{-}, w_{0}\right)\right\|_{B}+\left\|E_{0}\left(w_{+}, w_{-}, w_{0}\right)\right\|_{B}
$$

Proposition 10.5. Assume that $\sigma \in[0,20], u_{0}, U_{0}$ satisfy (10.19), w, w' $\mathbf{w}^{\prime} \in F^{\sigma}$, and $\psi: \mathbb{R} \rightarrow[0,1]$ is the smooth function defined in Section 5 . Then

$$
\begin{aligned}
\left\|\psi(t)\left[\mathbf{E}(\mathbf{w})-\mathbf{E}\left(\mathbf{w}^{\prime}\right)\right]\right\|_{N^{\sigma}} & \leq C\left\|\mathbf{w}-\mathbf{w}^{\prime}\right\|_{F^{\sigma}}\left(\delta+\|\mathbf{w}\|_{F^{0}}+\left\|\mathbf{w}^{\prime}\right\|_{F^{0}}\right) \\
& +C\left\|\mathbf{w}-\mathbf{w}^{\prime}\right\|_{F^{0}}\left(\|\mathbf{w}\|_{F^{\sigma}}+\left\|\mathbf{w}^{\prime}\right\|_{F^{\sigma}}\right) .
\end{aligned}
$$

Proof of Proposition 10.5. Let $T_{i,+}$ and $T_{i,-}, i \in\{1,2,3,4,5\}$, denote the terms in line $i$ in the formulas (2.11) and (2.13). To control $\left\|\psi(t)\left[T_{1,+}(\mathbf{w})-T_{1,+}\left(\mathbf{w}^{\prime}\right)\right]\right\|_{N^{\sigma}}$ it suffices to prove that

$$
\left\|m \cdot P_{\text {+high }}\left(\partial_{x}\left(m^{\prime} u v\right)\right)\right\|_{N^{\sigma}} \leq C\left(\|u\|_{F^{\sigma}}\|v\|_{F^{0}}+\|u\|_{F^{0}}\|v\|_{F^{\sigma}}\right),
$$

for any functions $u, v \in F^{\sigma}$, where $\|m\|_{S_{110}^{\infty}}=\left\|m^{\prime}\right\|_{S_{110}^{\infty}}=1$. We bound the lefthand side of (10.21) by

$$
\begin{aligned}
& \left\|\left(P_{\text {-high }}+P_{\text {low }}\right)\left[m \cdot P_{\text {+high }}\left(\partial_{x}\left(m^{\prime} u v\right)\right)\right]\right\|_{N^{\sigma}} \\
+ & \left\|P_{\text {+high }}\left[m \cdot\left(P_{- \text {high }}+P_{\text {low }}\right)\left(\partial_{x}\left(m^{\prime} u v\right)\right)\right]\right\|_{N^{\sigma}} \\
+ & \left\|P_{\text {+high }}\left[m \cdot\left(\partial_{x}\left(m^{\prime} u v\right)\right)\right]\right\|_{N^{\sigma}} .
\end{aligned}
$$

For the first two terms in (10.22) we use Lemma 10.4(a), Proposition 10.2, and (10.15). For the third term in (10.22) we use Proposition 10.2 and (9.3). The bound (10.21) follows.

To control $\left\|\psi(t)\left[T_{2,+}(\mathbf{w})-T_{2,+}\left(\mathbf{w}^{\prime}\right)\right]\right\|_{N^{\sigma}}$, it suffices to prove that

$\left\|m \cdot P_{+ \text {high }}\left[\partial_{x}\left(u_{0} \cdot P_{- \text {high }}\left(m^{\prime} u\right)\right)\right]\right\|_{N^{\sigma}}+\left\|m \cdot P_{\text {+high }}\left[\partial_{x}\left(u_{0} \cdot P_{\text {low }}\left(m^{\prime} u\right)\right)\right]\right\|\left\|_{N^{\sigma}} \leq C \delta\right\| u \|_{F^{\sigma}}$

for any $u \in F^{\sigma}$, where $\|m\|_{S_{110}^{\infty}}=\left\|m^{\prime}\right\|_{S_{110}^{\infty}}=1$. We use Lemma 10.3, Lemma 10.4(a), and (10.15). The first term in the left-hand side of (10.23) is dominated by

$$
\begin{aligned}
\left\|m \cdot P_{\text {+high }}\left[\partial_{x}\left(u_{0} \cdot P_{-\operatorname{high}}\left(m^{\prime} u\right)\right)\right]\right\|_{N_{6}^{\sigma}} & \leq C\left\|P_{+\operatorname{high}}\left[u_{0} \cdot P_{-\operatorname{high}}\left(m^{\prime} u\right)\right]\right\| \|_{F_{7}^{\sigma}} \\
& \leq C \delta\left\|m^{\prime} u\right\|_{F_{-10}^{\sigma}} \leq C \delta\|u\|_{F^{\sigma}},
\end{aligned}
$$

as desired. The bound for the second term is similar. Furthermore, the bound for $\left\|\psi(t)\left[T_{3,+}(\mathbf{w})-T_{3,+}\left(\mathbf{w}^{\prime}\right)\right]\right\|_{N^{\sigma}}$ follows in the same way.

To control $\left\|\psi(t)\left[T_{4,+}(\mathbf{w})-T_{4,+}\left(\mathbf{w}^{\prime}\right)\right]\right\|_{N^{\sigma}}$, it suffices to prove that

$$
\left\|\partial_{x}^{2} P_{-}\left[\left(\left(I-P_{0}\right) e^{i U_{0}}\right) \cdot P_{+h i g h}(m u)\right]\right\|_{N^{\sigma}} \leq C \delta\|u\|_{F^{\sigma}}
$$

for any $u \in F^{\sigma}$, where $\|m\|_{S_{110}^{\infty}}=1$. This follows as before, using Lemma 10.3. Lemma 10.4(b), (10.15), and the observation that $\left\|\left(I-P_{0}\right) e^{i U_{0}}\right\|_{S_{110}^{\infty}} \leq C \delta$. 
To control $\left\|\psi(t)\left[T_{5,+}(\mathbf{w})-T_{5,+}\left(\mathbf{w}^{\prime}\right)\right]\right\|_{N^{\sigma}}$, it suffices to prove that

$$
\left\|P_{+} \partial_{x} u_{0} \cdot u\right\|_{N^{\sigma}} \leq C \delta\|u\|_{F^{\sigma}}
$$

for any $u \in F^{\sigma}$. We bound the left-hand side of (10.25) by $(10.26)$

$\left\|\left(I-P_{0}\right)\left[\left(I-P_{0}\right)\left(P_{+} \partial_{x} u_{0}\right) u\right]\right\|_{N^{\sigma}}+\left\|\left(I-P_{0}\right)\left[\left(P_{0} P_{+} \partial_{x} u_{0}\right) u\right]\right\|_{N^{\sigma}}+\left\|P_{0}\left[P_{+} \partial_{x} u_{0} \cdot u\right]\right\|_{N^{\sigma}}$.

For the first term in (10.26) we use Proposition 10.2 with $m \equiv 1, m^{\prime} \equiv 0$. For the second term in (10.26) we use the bound (7.16). For the third term in (10.26) we use Lemma 10.3 .

$$
\left\|\left.P_{0}\left[P_{+} \partial_{x} u_{0} \cdot u\right]\right|_{N^{\sigma}} \leq C\right\| P_{+} \partial_{x} u_{0} \cdot u\left\|_{F_{-10}^{\sigma}} \leq C \delta\right\| u \|_{F_{-10}^{\sigma}},
$$

as desired.

The proofs for the terms $T_{i,-}$ are identical. To control $\left\|\psi(t)\left[E_{0}(\mathbf{w})-E_{0}\left(\mathbf{w}^{\prime}\right)\right]\right\|_{N^{\sigma}}$, it suffices to prove that

$$
\left\{\begin{array}{l}
\left\|P_{\text {low }} \partial_{x}(m u v)\right\|_{N^{\sigma}} \leq C\left(\|u\|_{F^{\sigma}}\|v\|_{F^{0}}+\|u\|_{F^{0}}\|v\|_{F^{\sigma}}\right) \\
\left\|P_{\text {low }} \partial_{x}\left(m u_{0} u\right)\right\|_{N^{\sigma}} \leq C \delta\|u\|_{F^{\sigma}}
\end{array}\right.
$$

for any functions $u, v \in F^{\sigma}$, where $\|m\|_{S_{15}^{\infty}}=1$. For the first bound in (10.27) we use Proposition 10.2. For the second bound we use Lemma 10.3 and the observation $\left\|m u_{0}\right\|_{S_{110}^{\infty}} \leq C \delta$. This completes the proof of Proposition 10.5 .

Proof of Theorem 1.1. For any interval $I \subseteq \mathbb{R}$ and $\sigma \geq 0$ we define the Banach spaces

$$
\left\{\begin{array}{l}
F^{\sigma}(I)=\left\{u \in \mathcal{S}^{\prime}(\mathbb{R} \times I):\|u\|_{F^{\sigma}(I)}:=\inf _{\widetilde{u} \equiv u \text { on } \mathbb{R} \times I}\|\widetilde{u}\|_{F^{\sigma}}<\infty\right\} \\
N^{\sigma}(I)=\left\{u \in \mathcal{S}^{\prime}(\mathbb{R} \times I):\|u\|_{N^{\sigma}(I)}:=\inf _{\widetilde{u} \equiv u \text { on } \mathbb{R} \times I}\|\widetilde{u}\|_{N^{\sigma}}<\infty\right\} .
\end{array}\right.
$$

With this notation, the estimates in Lemma 5.1 and Lemma 5.2 become

$$
\left\|W\left(t-t_{0}\right) \phi\right\|_{F^{\sigma}\left(\left[t_{0}-a, t_{0}+a\right]\right)} \leq C\|\phi\|_{\widetilde{H}^{\sigma}}
$$

and

$$
\left\|\int_{t_{0}}^{t} W(t-s)(u(s)) d s\right\|_{F^{\sigma}\left(\left[t_{0}-a, t_{0}+a\right]\right)} \leq C\|u\|_{N^{\sigma}\left(\left[t_{0}-a, t_{0}+a\right]\right)},
$$

for any $\sigma \in[0,20], t_{0} \in \mathbb{R}$ and $a \in[0,5 / 4]$. The estimate in Proposition 10.5 becomes

$$
\begin{aligned}
\left\|\mathbf{E}(\mathbf{w})-\mathbf{E}\left(\mathbf{w}^{\prime}\right)\right\|_{N^{\sigma}(I)} & \leq C\left\|\mathbf{w}-\mathbf{w}^{\prime}\right\|_{F^{\sigma}(I)}\left(\delta+\|\mathbf{w}\|_{F^{0}(I)}+\left\|\mathbf{w}^{\prime}\right\|_{F^{0}(I)}\right) \\
& +C\left\|\mathbf{w}-\mathbf{w}^{\prime}\right\|_{F^{0}(I)}\left(\|\mathbf{w}\|_{F^{\sigma}(I)}+\left\|\mathbf{w}^{\prime}\right\|_{F^{\sigma}(I)}\right),
\end{aligned}
$$

for any $\sigma \in[0,20]$ and $I \subseteq[-5 / 4,5 / 4]$, provided that (10.19) holds.

Assume that $u_{0}, U_{0}$ are fixed and satisfy (10.19). For data $\Phi=\left(\phi_{+}, \phi_{-}, \phi_{0}\right) \in$ $\widetilde{H}^{20}$ with the property

$$
\|\left.\Phi\right|_{\widetilde{H}^{0}} \leq \delta
$$

we consider the vector-valued initial-value problem

$$
\left\{\begin{array}{l}
\left(\partial_{t}+\mathcal{H} \partial_{x}^{2}\right) \mathbf{v}=\mathbf{E}(\mathbf{v}) \text { on } \mathbb{R} \times[-5 / 4,5 / 4] \\
\mathbf{v}(0)=\Phi
\end{array}\right.
$$


We can construct a solution of (10.32) by iteration: let $\mathbf{v}^{0}=(0,0,0)$ and let

$$
\mathbf{v}^{k+1}=W(t) \Phi+\int_{0}^{t} W(t-s)\left(\mathbf{E}\left(\mathbf{v}^{k}\right)(s)\right) d s, k=0,1, \ldots
$$

In view of (10.28), (10.29), (10.30), and (10.31), $\left\|\mathbf{v}^{k}\right\|_{F^{0}([-5 / 4,5 / 4])} \leq C \delta$ for any $k \geq 0$. Thus, using (10.28), (10.29), (10.30), and (10.31) again,

$$
\left\|\mathbf{v}^{k+1}-\mathbf{v}^{k}\right\|_{F^{0}([-5 / 4,5 / 4])} \leq(C \delta)^{k+1} \text { for any } k=0,1, \ldots
$$

Using (10.28), (10.29), (10.30), (10.31), and (10.34), we obtain $\|\left.\mathbf{v}^{k}\right|_{F^{\sigma}([-5 / 4,5 / 4])} \leq$ $C\|\Phi\|_{\widetilde{H}^{\sigma}}, \sigma \in[0,20]$, and then

$$
\left\|\mathbf{v}^{k+1}-\mathbf{v}^{k}\right\|_{F^{\sigma}([-5 / 4,5 / 4])} \leq(C \delta)^{k}|| \Phi \|_{\widetilde{H}^{\sigma}} \text { for any } k=0,1, \ldots
$$

Thus the sequence $\mathbf{v}^{k}$ converges in the space $F^{20}([-5 / 4,5 / 4])$ to a function $\mathbf{v}=$ $\mathbf{v}(\Phi)$. In addition, for any $\sigma \in[0,20]$,

$$
\|\mathbf{v}(\Phi)\|_{F^{\sigma}([-5 / 4,5 / 4])} \leq C\|\Phi\|_{\widetilde{H}^{\sigma}},
$$

$\mathbf{v}(\Phi) \in C\left([-5 / 4,5 / 4]: \widetilde{H}^{20}\right)$ (using (4.10) $), \mathbf{v}(\Phi)$ solves the initial-value problem (10.32), and if $\|\Phi\|_{\widetilde{H}^{0}},\left\|\Phi^{\prime}\right\|_{\widetilde{H}^{0}} \leq \delta$, then

$$
\begin{aligned}
& \left\|\mathbf{v}(\Phi)-\mathbf{v}\left(\Phi^{\prime}\right)\right\|_{F^{\sigma}([-5 / 4,5 / 4])} \\
& \leq C\left\|\Phi-\Phi^{\prime}\right\|_{\widetilde{H}^{\sigma}}+C\left(\|\Phi\|_{\widetilde{H}^{\sigma}}+\left\|\Phi^{\prime}\right\|_{\widetilde{H}^{\sigma}}\right)\left\|\mathbf{v}(\Phi)-\mathbf{v}\left(\Phi^{\prime}\right)\right\|_{F^{0}([-5 / 4,5 / 4])} .
\end{aligned}
$$

In particular, when $\sigma=0,\left\|\mathbf{v}(\Phi)-\mathbf{v}\left(\Phi^{\prime}\right)\right\|_{F^{0}([-5 / 4,5 / 4])} \leq C\left\|\Phi-\Phi^{\prime}\right\|_{\widetilde{H}^{0}}$.

Assume now that we start with data $\phi \in H_{r}^{\infty}$ with the property

$$
\|\phi\|_{L^{2}} \leq \delta_{0}=\delta / C \text {, where } C \text { is sufficiently large. }
$$

We construct the functions $u_{0}, \widetilde{u}, U_{0}, \mathbf{w}=\left(w_{+}, w_{-}, w_{0}\right)$, and

$$
\Phi=\left(\phi_{+}, \phi_{-}, \phi_{0}\right)=\left(e^{i U_{0}(., 0)} P_{+ \text {high }} \phi, e^{-i U_{0}(., 0)} P_{- \text {high }} \phi, 0\right)
$$

as in Section 2. Clearly, (10.31) holds due to Lemma 10.1, and $\Phi \in \widetilde{H}^{20}$. We show now that

$$
\mathbf{w} \equiv \mathbf{v}(\Phi) \text { in } \mathbb{R} \times[-1,1]
$$

where $\mathbf{v}(\Phi)$ is constructed as before. This is somewhat delicate since it is not clear how to show algebraically that the function $e^{-i U_{0}} v_{+}+e^{i U_{0}} v_{-}+v_{0}+u_{0}$ is a solution of the original initial-value problem.

To prove (10.38), we show first that

$$
\|\mathbf{w}(t)\|_{\widetilde{H}^{0}} \leq C \delta_{0} \text { for any } t \in[-5 / 4,5 / 4] .
$$

For the functions $w_{+}$and $w_{-}$this follows directly using the definition (2.5) and Lemma 10.1, since, in view of the conservation law (1.2),

$$
\|\widetilde{u}\|_{L_{t}^{\infty} L_{x}^{2}}+\left\|u_{0}\right\|_{L_{t}^{\infty} L_{x}^{2}} \leq 3 \delta_{0} \text { for any } t \in[-5 / 4,5 / 4] .
$$

To prove (10.39) for the function $w_{0}$, we use first the definition (2.5) and (10.40), so it suffices to prove that

$$
\left\|\eta_{0}(\xi) \mathcal{F}_{1}\left(w_{0}(t)\right)(\xi)\right\|_{B_{0}} \leq C \delta_{0}, t \in[-5 / 4,5 / 4] .
$$

For this we use the equation (2.4) (notice $\left.w_{0}(0) \equiv 0\right)$. It suffices to prove that $(10.42)$

$$
\left\|\eta_{0}(\xi) \xi^{2} \operatorname{sgn}(\xi) \mathcal{F}_{1}(\widetilde{u}(t))(\xi)\right\|_{B_{0}}+\left\|\eta_{0}(\xi) \xi \mathcal{F}_{1}\left(\widetilde{u}(t)\left(\widetilde{u}(t) / 2+u_{0}(t)\right)\right)(\xi)\right\|_{B_{0}} \leq C \delta_{0},
$$


for any $t \in[-5 / 4,5 / 4]$. We bound the first term in (10.42) by

$$
\sum_{k^{\prime} \leq 1} 2^{-k^{\prime}}\left\|\chi_{k^{\prime}}(\xi) \xi^{2} \mathcal{F}_{1}(\widetilde{u}(t))(\xi)\right\|_{L_{\xi}^{2}} \leq C\|\widetilde{u}(t)\|_{L_{x}^{2}} \leq C \delta_{0},
$$

as desired. We bound the second term in (10.42) by

$$
\left\|\left.\mathcal{F}_{1}^{-1}\left[\eta_{0}(\xi) \xi \mathcal{F}_{1}\left(\widetilde{u}(t)\left(\widetilde{u}(t) / 2+u_{0}(t)\right)\right)(\xi)\right]\right|_{L_{x}^{1}} \leq C\right\| \widetilde{u}(t)\left\|_{L_{x}^{2}}\right\| \widetilde{u}(t) / 2+u_{0}(t) \|_{L_{x}^{2}},
$$

which suffices in view of (10.40). This completes the proof of (10.39).

Next, we show that there is $\varepsilon=\varepsilon\left(\|\phi\|_{H^{100}}\right)$ with the property that

$$
\|\mathbf{w}\|_{F^{0}\left(\left[t_{0}-\varepsilon, t_{0}+\varepsilon\right]\right)} \leq C \delta_{0} \text { for any } t_{0} \in[-1,1] .
$$

Let $\mathbf{g}=\psi(t)\left(\partial_{t}+\mathcal{H} \partial_{x}^{2}\right) \mathbf{w}$. In view of (10.28), (10.29), (10.39), and (10.15), for (10.43) it suffices to prove that

$$
\left\|\psi\left(\left(t-t_{0}\right) / \varepsilon\right) \cdot \mathbf{g}\right\|_{N_{6}^{0}} \leq C\left(\|\phi\|_{H^{100}}\right) \varepsilon^{1 / 4} .
$$

We show first that for any $t \in[-5 / 4,5 / 4]$

$$
\left\|\left(I-\partial_{t}^{2}\right) \mathbf{g}(t)\right\|_{\widetilde{H}^{20}} \leq C\left(\|\phi\|_{H^{100}}\right) .
$$

For (10.45) we notice first that $\mathcal{H} \partial_{x}^{2}: \widetilde{H}^{\sigma} \rightarrow \widetilde{H}^{\sigma-2}$ is a bounded operator. Thus it suffices to prove that $\left\|\partial_{t}^{\sigma} \mathbf{w}\right\|_{\widetilde{H}^{50}} \leq C\left(\|\phi\|_{H^{100}}\right), \sigma=0,1,2,3$. For $w_{+}$and $w_{-}$ this is clear using the definitions $w_{ \pm}=e^{ \pm i U_{0}} P_{ \pm \text {high }} \widetilde{u}$ and Lemma 10.1. For $w_{0}$ this follows using the identity (2.4)

$$
\partial_{t} w_{0}=-\mathcal{H} \partial_{x}^{2} w_{0}-P_{\text {low }} \partial_{x}\left(\left(u_{0}+\widetilde{u} / 2\right) \cdot \widetilde{u}\right),
$$

the bound (10.39), and the same argument as in the proof of (10.41). This completes the proof of (10.45). To pass from (10.45) to (10.44), we may assume $t_{0}=0$ and $\mathbf{g}=g$ is scalar valued. It suffices to prove that

$$
\|\psi(t / \varepsilon) \cdot g\|_{N_{6}^{0}} \leq C \varepsilon^{1 / 4}\left\|\left(I-\partial_{t}^{2}\right) g\right\|_{L_{t}^{1} \widetilde{H}^{20}} .
$$

In view of the $L_{t}^{1}$ norm in the right-hand side of (10.46), we may assume that $g(x, t)=h(x) K\left(t-t_{0}\right)$, where $K(t)=\int_{\mathbb{R}}\left(\tau^{2}+1\right)^{-1} e^{i t \tau} d \tau$ and $\left\|\left(I-\partial_{t}^{2}\right) g\right\|_{L_{t}^{1} \widetilde{H}^{20}} \approx$ $\|h \mid\|_{\widetilde{H}^{20}}$. The bound (10.46) then follows easily from the definitions.

We can now complete the proof of (10.38). Assume $\mathbf{w}\left(t_{0}\right)=\mathbf{v}\left(t_{0}\right)=\Psi$ for some $t_{0} \in[-1,1]$. Then, for $t \in\left[t_{0}-\varepsilon, t_{0}+\varepsilon\right]$ we write

$$
\left\{\begin{array}{l}
\mathbf{w}(t)=W\left(t-t_{0}\right) \Psi+\int_{t_{0}}^{t} W(t-s)(\mathbf{E}(\mathbf{w})(s)) d s \\
\mathbf{v}(t)=W\left(t-t_{0}\right) \Psi+\int_{t_{0}}^{t} W(t-s)(\mathbf{E}(\mathbf{v})(s)) d s .
\end{array}\right.
$$

We subtract the two identities and use (10.29), (10.30), (10.35) (all with $\sigma=0$ ), and (10.43). The result is

$\|\mathbf{v}-\mathbf{w}\|_{F^{0}\left(\left[t_{0}-\varepsilon, t_{0}+\varepsilon\right]\right)} \leq C\|\mathbf{E}(\mathbf{v})-\mathbf{E}(\mathbf{w})\|_{N^{0}\left(\left[t_{0}-\varepsilon, t_{0}+\varepsilon\right]\right)} \leq C \delta\|\mathbf{v}-\mathbf{w}\|_{F^{0}\left(\left[t_{0}-\varepsilon, t_{0}+\varepsilon\right]\right)}$.

So $\mathbf{v} \equiv \mathbf{w}$ in $\mathbf{R} \times\left[t_{0}-\varepsilon, t_{0}+\varepsilon\right]$. Since $\mathbf{w}(0)=\mathbf{v}(0)=\Phi$, this suffices to prove (10.38).

We prove now part (a) of the theorem. Assume that

$$
\phi_{n} \in H_{r}^{\infty} \text { and } \lim _{n \rightarrow \infty} \phi_{n}=\phi \text { in } L^{2} .
$$


By rescaling 3 , we may assume $\|\phi\|_{L^{2}} \leq \delta_{0} / 2$, as in (10.37). By using the conservation law (1.2), we may assume $T=1$. It suffices to prove that for any $\epsilon>0$

$$
\left\|S_{1}^{\infty}\left(\phi_{n}\right)-S_{1}^{\infty}\left(\phi_{m}\right)\right\|_{L_{t}^{\infty} L_{x}^{2}} \leq \varepsilon \text { for } m, n \text { sufficiently large. }
$$

We fix $M=M(\phi, \varepsilon)$ sufficiently large and define $\widehat{\phi^{M}}(\xi)=\mathbf{1}_{[-M, M]}(\xi) \widehat{\phi}(\xi)$ and $\widehat{\phi_{n}^{M}}(\xi)=\mathbf{1}_{[-M, M]}(\xi) \widehat{\phi_{n}}(\xi)$. It is known that the flow map $S_{1}^{\infty}$ extends continuously on, say, $H_{r}^{2}$ (see, for example, [17]). Since $\lim _{n \rightarrow \infty} \phi_{n}^{M}=\phi^{M}$ in $H_{r}^{2}$,

$$
\lim _{n, m \rightarrow \infty}\left\|S_{1}^{\infty}\left(\phi_{n}^{M}\right)-S_{1}^{\infty}\left(\phi_{m}^{M}\right)\right\|_{L_{t}^{\infty} H_{x}^{2}}=0 .
$$

We estimate now $\left\|S_{1}^{\infty}\left(\phi_{n}\right)-S_{1}^{\infty}\left(\phi_{n}^{M}\right)\right\|_{L_{t}^{\infty} L_{x}^{2}}$. As in Section 2, we construct $u_{0, n}$, $U_{0, n}$ (which are identical for both functions $\phi_{n}$ and $\phi_{n}^{M}$ ),

$$
\Phi_{n}=\left(e^{i U_{0, n}} P_{+ \text {high }} \phi_{n}, e^{-i U_{0, n}} P_{- \text {high }} \phi_{n}, 0\right)
$$

and

$$
\Phi_{n}^{M}=\left(e^{i U_{0, n}} P_{+\mathrm{high}} \phi_{n}^{M}, e^{-i U_{0, n}} P_{-\mathrm{high}} \phi_{n}^{M}, 0\right) .
$$

Using Lemma 2.1, the identity (10.38), (10.36) with $\sigma=0$, (4.10), and Lemma 10.1 .

$$
\begin{aligned}
\left\|S_{1}^{\infty}\left(\phi_{n}\right)-S_{1}^{\infty}\left(\phi_{n}^{M}\right)\right\|_{L_{t}^{\infty} L_{x}^{2}} & \leq C\left\|\mathbf{v}\left(\Phi_{n}\right)-\mathbf{v}\left(\Phi_{n}^{M}\right)\right\|_{L_{t \in[-1,1]}^{\infty} L_{x}^{2}} \leq C\left\|\Phi_{n}-\Phi_{n}^{M}\right\|_{\widetilde{H}^{0}} \\
& \leq C\left\|\phi_{n}-\phi_{n}^{M}\right\|_{L^{2}} \leq C\left(\left\|\phi-\phi^{M}\right\|_{L^{2}}+\left\|\phi-\phi_{n}\right\|_{L^{2}}\right) .
\end{aligned}
$$

The bound (10.47) follows if $M=M(\phi, \varepsilon)$ and $n$ are sufficiently large.

For part (b) of the theorem, we may assume that $\sigma \leq 2$. The same argument as before works, once we observe that, using (10.36),

$$
\left\|\mathbf{v}\left(\Phi_{n}\right)-\mathbf{v}\left(\Phi_{n}^{M}\right)\right\|_{F^{\sigma}([-5 / 4,5 / 4])} \leq C\left\|\Phi_{n}-\Phi_{n}^{M}\right\|_{\widetilde{H}^{\sigma}}\left(1+\left\|\Phi_{n}\right\|_{\widetilde{H}^{\sigma}}+\left\|\Phi_{n}^{M}\right\|_{\widetilde{H}^{\sigma}}\right) .
$$

\section{ACKNOWLEDGMENTS}

We would like to thank S. Herr and H. Koch for useful discussions in the early stages of this work.

\section{REFERENCES}

[1] T. B. Benjamin, Internal waves of permanent form in fluids of great depth, J. Fluid Mech. 29 (1967), 559-592.

[2] J. Bourgain, Fourier transform restriction phenomena for certain lattice subsets and applications to nonlinear evolution equations II. The KdV-equation. Geom. Funct. Anal. 3 (1993), 209-262. MR1215780 (95d:35160b)

[3] N. Burq and F. Planchon, On well-posedness for the Benjamin-Ono equation, preprint (2005).

[4] J. Colliander, C. E. Kenig, and G. Staffilani, Local well-posedness for dispersion-generalized Benjamin-Ono equations, Differential Integral Equations 16 (2003), 1441-1472. MR2029909 (2005a:35240)

[5] J. Ginibre and G. Velo, Smoothing properties and existence of solutions for the generalized Benjamin-Ono equation, J. Differential Equations 93 (1991), 150-232. MR1122309 (93b:35116)

[6] A. D. Ionescu and C. E. Kenig, Complex-valued solutions of the Benjamin-Ono equation, preprint (2006).

\footnotetext{
${ }^{3}$ The smooth flow has invariance property $S^{\infty}\left(\phi_{\lambda}\right)=\left[S^{\infty}(\phi)\right]_{\lambda}$, where $\phi_{\lambda}(x)=\lambda \phi(\lambda x)$ and $u_{\lambda}(x, t)=\lambda u\left(\lambda x, \lambda^{2} t\right)$.
} 
[7] R. J. Iorio, On the Cauchy problem for the Benjamin-Ono equation, Comm. Partial Differential Equations 11 (1986), 1031-1081. MR0847994 (88b:35034)

[8] C. E. Kenig and K. D. Koenig, On the local well-posedness of the Benjamin-Ono and modified Benjamin-Ono equations, Math. Res. Lett. 10 (2003), 879-895. MR2025062 (2004j:35249)

[9] C. E. Kenig, G. Ponce, and L. Vega, Well-posedness of the initial value problem for the Korteweg-de Vries equation, J. Amer. Math. Soc. 4 (1991), 323-347. MR1086966|(92c:35106)

[10] S. Klainerman and M. Machedon, Space-time estimates for null forms and the local existence theorem, Comm. Pure Appl. Math. 46 (1993), 1221-1268. MR1231427 (94h:35137)

[11] S. Klainerman and S. Selberg, Remark on the optimal regularity for equations of wave maps type, Comm. Partial Differential Equations 22 (1997), 901-918. MR.1452172 (99c:35163)

[12] H. Koch and N. Tzvetkov, On the local well-posedness of the Benjamin-Ono equation in $H^{s}(\mathbb{R})$, Int. Math. Res. Not. 2003 (2003), 1449-1464. MR1976047(2004b:35284)

[13] H. Koch and N. Tzvetkov, Nonlinear wave interactions for the Benjamin-Ono equation, Int. Math. Res. Not. 2005 (2005), 1833-1847. MR2172940 (2006f:35245)

[14] L. Molinet, J.-C. Saut, and N. Tzvetkov, Ill-posedness issues for the Benjamin-Ono and related equations, SIAM J. Math. Anal. 33 (2001), 928-988. MR.1885293 (2002k:35281)

[15] L. Molinet, Global well-posedness in $L^{2}$ for the periodic Benjamin-Ono equation, preprint (2006).

[16] H. Ono, Algebraic solitary waves in stratified fluids, J. Phys. Soc. Japan 39 (1975), 1082 1091. MR0398275 (53:2129)

[17] G. Ponce, On the global well-posedness of the Benjamin-Ono equation, Differential Integral Equations 4 (1991), 527-542. MR.1097916 (92e:35137)

[18] J.-C. Saut, Sur quelques généralisations de l'équation de Korteweg-de Vries, J. Math. Pures Appl. 58 (1979), 21-61. MR0533234 (82m:35133)

[19] T. Tao, Global regularity of wave maps I. Small critical Sobolev norm in high dimension, Int. Math. Res. Not. 2001 (2001), 299-328. MR1820329 (2001m:35200)

[20] T. Tao, Global regularity of wave maps II. Small energy in two dimensions, Comm. Math. Phys. 224 (2001), 443-544. MR.1869874(2002h:58052)

[21] T. Tao, Multilinear weighted convolution of $L^{2}$ functions, and applications to nonlinear dispersive equations, Amer. J. Math. 123 (2001), 839-908. MR.1854113 (2002k:35283)

[22] T. Tao, Global well-posedness of the Benjamin-Ono equation in $H^{1}(\mathbb{R})$, J. Hyperbolic Differ. Equ. 1 (2004), 27-49. MR2052470 (2005f:35273)

[23] D. Tataru, Local and global results for wave maps I, Comm. Partial Differential Equations 23 (1998), 1781-1793. MR.1641721 (99j:58209)

[24] D. Tataru, On global existence and scattering for the wave maps equation, Amer. J. Math. 123 (2001), 37-77. MR1827277 (2002c:58045)

[25] M. Tom, Smoothing properties of some weak solutions to the Benjamin-Ono equation, Differential Integral Equations 3 (1990), 683-694. MR.1044213 (91e:35191)

Department of Mathematics, University of Wisconsin, 480 Lincoln Drive, Van Vleck Hall, Madison, Wisconsin 53706

E-mail address: ionescu@math.wisc.edu

Department of Mathematics, University of Chicago, 5734 University Ave, Chicago, ILLINOIS 60637-1514

E-mail address: cek@math.uchicago.edu 